\title{
Strongly Luminescent Pt(IV) Complexes with a Mesoionic $N$-Heterocyclic Carbene Ligand: Tuning their Photophysical Properties
}

Ángela Vivancos, ${ }^{\dagger}$ Adrián Jiménez-García, ${ }^{\dagger}$ Delia Bautista ${ }^{\ddagger}$ and Pablo González-Herrero*,†

†Departamento de Química Inorgánica, Facultad de Química, Universidad de Murcia, Campus de Espinardo, 19, 30100 Murcia, Spain.

*Área Científica y Técnica de Investigación, Universidad de Murcia, Campus de Espinardo, 21, 30100 Murcia, Spain.

\section{Contents:}

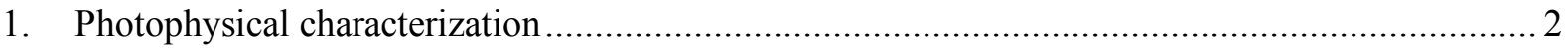

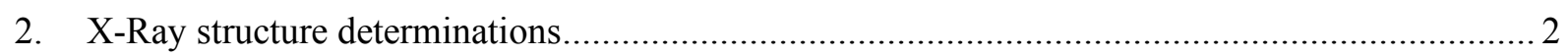

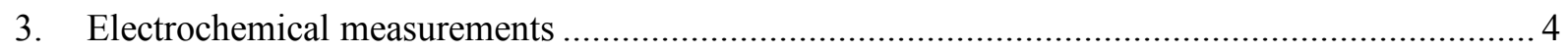

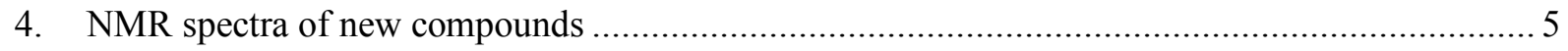

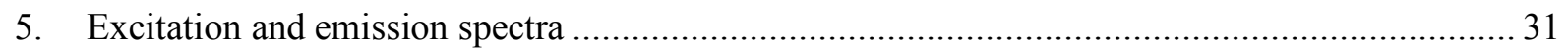

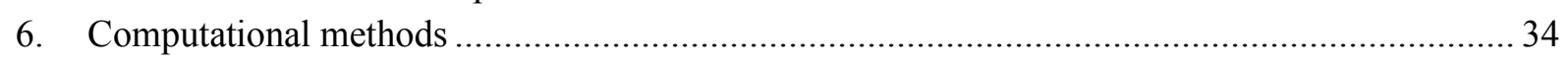

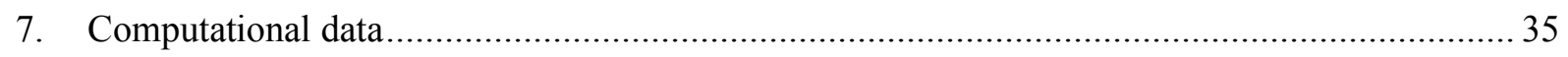

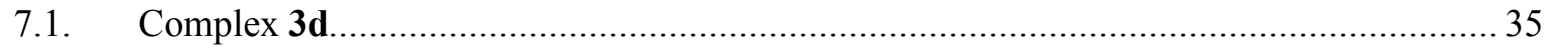

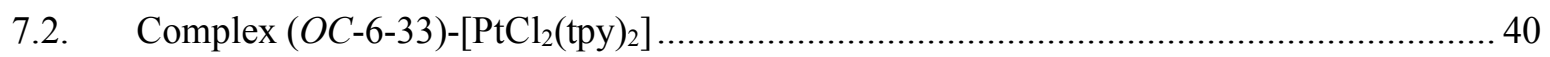

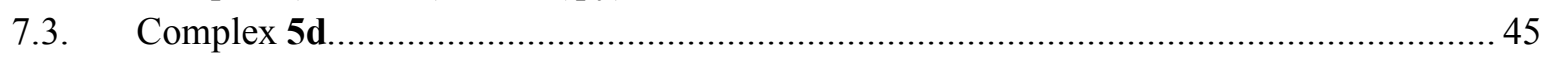

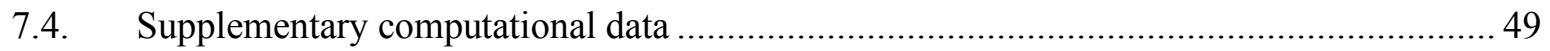

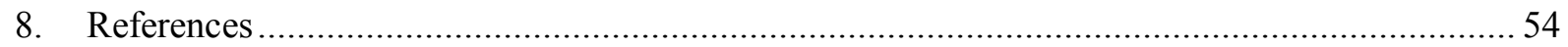




\section{Photophysical characterization}

UV-vis absorption spectra were recorded on a Perkin-Elmer Lambda 750S spectrophotometer. Excitation and emission spectra were recorded on a Jobin Yvon Fluorolog 3-22 spectrofluorometer. Emission data in PMMA matrices were registered using quartz slides as sample holders. Lifetimes were determined with an IBH FluoroHub controller in MCS mode and the Fluorolog's FL-1040 phosphorimeter pulsed xenon lamp as excitation source; the estimated uncertainty is $\pm 10 \%$ or better. Emission quantum yields $(\Phi)$ were determined using a Hamamatsu C11347 Absolute PL Quantum Yield Spectrometer; the estimated uncertainty is $\pm 5 \%$ or better. Unless otherwise specified, emission data were obtained under rigorous exclusion of oxygen, by bubbling argon through the solutions for $30 \mathrm{~min}$ or placing the PMMA films under argon.

\section{X-Ray structure determinations}

Single crystals of cis-C, $C^{*}-\mathbf{2 d}, \mathbf{3 d} \cdot \mathrm{CH}_{2} \mathrm{Cl}_{2}, \mathbf{3 e}, \mathbf{5 a}$ and $\mathbf{5 d}$ suitable for X-ray diffraction were obtained by the liquid-liquid diffusion method from $\mathrm{CH}_{2} \mathrm{Cl}_{2} / \mathrm{Et}_{2} \mathrm{O}$. The data were collected on a Bruker D8 QUEST diffractometer with monochromated Mo-K $\alpha$ radiation performing $\varphi$ and $\omega$ scans. The structures were solved by direct methods and refined anisotropically on $F^{2}$ using the program SHELXL2018. ${ }^{1}$ Methyl hydrogens were included as part of rigid idealized methyl groups allowed to rotate but not tip; other hydrogens were included using a riding model. Numerical details are given in the Supporting Information (Table S1). Special features of refinement: In 3d, the structure contains one resolved molecule of dichloromethane that is disordered over two positions, ca 55:45\%. The butyl fragment is disordered over two positions with a ca. 54:46\% occupancy distribution. In 3e, the butyl ligand is disordered over two positions, 79:21\%; the structure contains a poorly-resolved region of residual electron density, which could not be adequately modelled and therefore was "removed" using the program SQUEEZE, ${ }^{2,3}$ which is part of the PLATON system. The void volume per cell was $136 \AA^{3}$, with a void electron count per cell of 112. This additional solvent was not taken account of when calculating derived parameters such as the formula weight, because the nature of the solvent was uncertain. 
Table S1. Crystallographic data for $c i s-C, C^{*}-\mathbf{2 d}, \mathbf{3 d} \cdot \mathrm{CH}_{2} \mathrm{Cl}_{2}, \mathbf{3 e}, \mathbf{5 a}$ and $\mathbf{5 d}$.

\begin{tabular}{|c|c|c|c|c|c|}
\hline & $c i s-C, C^{*}-\mathbf{2 d}$ & 3d $\cdot \mathrm{CH}_{2} \mathrm{Cl}_{2}$ & $3 \mathbf{e}$ & $5 \mathbf{a}$ & $5 d$ \\
\hline formula & $\mathrm{C}_{25} \mathrm{H}_{27} \mathrm{ClN}_{4} \mathrm{Pt}$ & $\mathrm{C}_{26} \mathrm{H}_{28} \mathrm{Cl}_{4} \mathrm{~N}_{4} \mathrm{Pt}$ & $\mathrm{C}_{22} \mathrm{H}_{22} \mathrm{Cl}_{2} \mathrm{~N}_{4} \mathrm{PtS}$ & $\mathrm{C}_{22} \mathrm{H}_{23} \mathrm{Cl}_{2} \mathrm{~N}_{5} \mathrm{Pt}$ & $\mathrm{C}_{25} \mathrm{H}_{26} \mathrm{Cl}_{2} \mathrm{~N}_{4} \mathrm{Pt}$ \\
\hline fw & 614.04 & 733.41 & 640.48 & 623.44 & 648.49 \\
\hline$T(\mathrm{~K})$ & $100(2)$ & $100(2)$ & $100(2)$ & $100(2)$ & $100(2)$ \\
\hline$\lambda$ & 0.71073 & 0.71073 & 0.71073 & 0.71073 & 0.71073 \\
\hline cryst syst & Monoclinic & Triclinic & Triclinic & Monoclinic & Monoclinic \\
\hline space group & $\mathrm{P} 21 / \mathrm{c}$ & P-1 & P-1 & $\mathrm{P} 2{ }_{1} / \mathrm{n}$ & $\mathrm{P} 2{ }_{1} / \mathrm{c}$ \\
\hline$a(\AA)$ & $15.308(2)$ & $8.1545(10)$ & $8.0245(4)$ & $11.4100(14)$ & $13.1655(11)$ \\
\hline$b(\AA)$ & $9.8691(13)$ & $10.9536(14)$ & $10.9249(5)$ & $12.5588(16)$ & $11.8515(10)$ \\
\hline$c(\AA)$ & $17.150(3)$ & $16.1105(19)$ & $15.4363(7)$ & $30.859(4)$ & $16.5800(14)$ \\
\hline$\alpha\left(^{\circ}\right)$ & 90 & $107.517(4)$ & $74.001(2)$ & 90 & 90 \\
\hline$\beta\left(^{\circ}\right)$ & $115.884(4)$ & $92.117(4)$ & $88.469(2)$ & $100.025(4)$ & $111.814(4)$ \\
\hline$\gamma\left({ }^{\circ}\right)$ & 90 & $102.156(4)$ & $75.476(2)$ & 90 & 90 \\
\hline$V\left(\AA^{3}\right)$ & $2331.0(6)$ & $1333.7(3)$ & $1257.96(10)$ & $4354.5(9)$ & $2401.8(4)$ \\
\hline$Z$ & 4 & 2 & 2 & 8 & 4 \\
\hline $\begin{array}{c}\rho_{\text {calcd }}(\mathrm{Mg} \\
\left.\mathrm{m}^{-3}\right)\end{array}$ & 1.750 & 1.826 & 1.691 & 1.902 & 1.793 \\
\hline$\mu\left(\mathrm{mm}^{-1}\right)$ & 6.154 & 5.684 & 5.888 & 6.710 & 6.085 \\
\hline $\mathrm{R}^{a}{ }^{a}$ & 0.0155 & 0.0204 & 0.0182 & 0.0230 & 0.0142 \\
\hline $\mathrm{wR} 2^{b}$ & 0.0350 & 0.0499 & 0.0476 & 0.0440 & 0.0300 \\
\hline
\end{tabular}

${ }^{a} \mathrm{R} 1=\Sigma|| F_{\mathrm{o}}|-| F_{\mathrm{c}}|| \Sigma\left|F_{\mathrm{o}}\right|$ for reflections with $I>2 \sigma(I) .{ }^{b} \mathrm{wR} 2=\left[\Sigma\left[w\left(F_{\mathrm{o}}{ }^{2}-F_{\mathrm{c}}{ }^{2}\right)^{2} / \Sigma\left[w\left(F_{\mathrm{o}}{ }^{2}\right)^{2}\right]\right]^{0.5}\right.$ for all reflections; $w^{-1}$ $=\sigma^{2}\left(F^{2}\right)+(a P)^{2}+b P$, where $P=\left(2 F_{\mathrm{c}}^{2}+F_{\mathrm{o}}^{2}\right) / 3$ and $a$ and $b$ are constants set by the program. 


\section{Electrochemical measurements}

Cyclic voltammograms were registered with a potentiostat/galvanostat AUTOLAB-100 (EchoChemie, Utrecht), employing a three-electrode electrochemical cell equipped with a glassy carbon working electrode (Metrohm, $2 \mathrm{~mm}$ diameter), an $\mathrm{Ag} / \mathrm{AgCl} / 3 \mathrm{M} \mathrm{KCl}$ electrode reference, and a glassy carbon rod counter electrode. The measurements were carried out at $298 \mathrm{~K}$ under an argon atmosphere, using degassed $1 \mathrm{mM}$ solutions of the complexes in extra-dry MeCN (Acros Organics) and $0.1 \mathrm{M}$ $\left(\mathrm{Bu}_{4} \mathrm{~N}\right) \mathrm{PF}_{6}$ as the electrolyte. Before each experiment, the working electrode was polished with alumina slurry $(0.05 \mu \mathrm{m})$ and rinsed with water and acetone. The electrodes were activated electrochemically in the background solution by means of several voltammetric cycles at $1 \mathrm{~V} \mathrm{~s}^{-1}$ between $-2.8 \mathrm{~V}$ and $2.2 \mathrm{~V}$. At the end of each experiment, the reference electrode was checked against the $\mathrm{Fc}^{+} / \mathrm{Fc}$ redox couple. Potentials are given against the standard calomel electrode (SCE).

The HOMO/LUMO energies were estimated from the onset values of the oxidation and reduction waves, respectively, referenced against $\mathrm{Fc}^{+} / \mathrm{Fc}(0.40 \mathrm{~V}$ vs SCE in $\mathrm{MeCN})$, using a formal potential of $5.1 \mathrm{eV}$ for the $\mathrm{Fc}^{+} / \mathrm{Fc}$ couple in the Fermi scale: ${ }^{4}$

$$
E_{\mathrm{HOMO}}=-\left(E_{\text {onset,ox }}+5.1-0.4\right) \mathrm{eV} ; E_{\mathrm{LUMO}}=-\left(E_{\text {onset, } r e d}+5.1-0.4\right) \mathrm{eV}
$$




\section{NMR spectra of new compounds}

侘

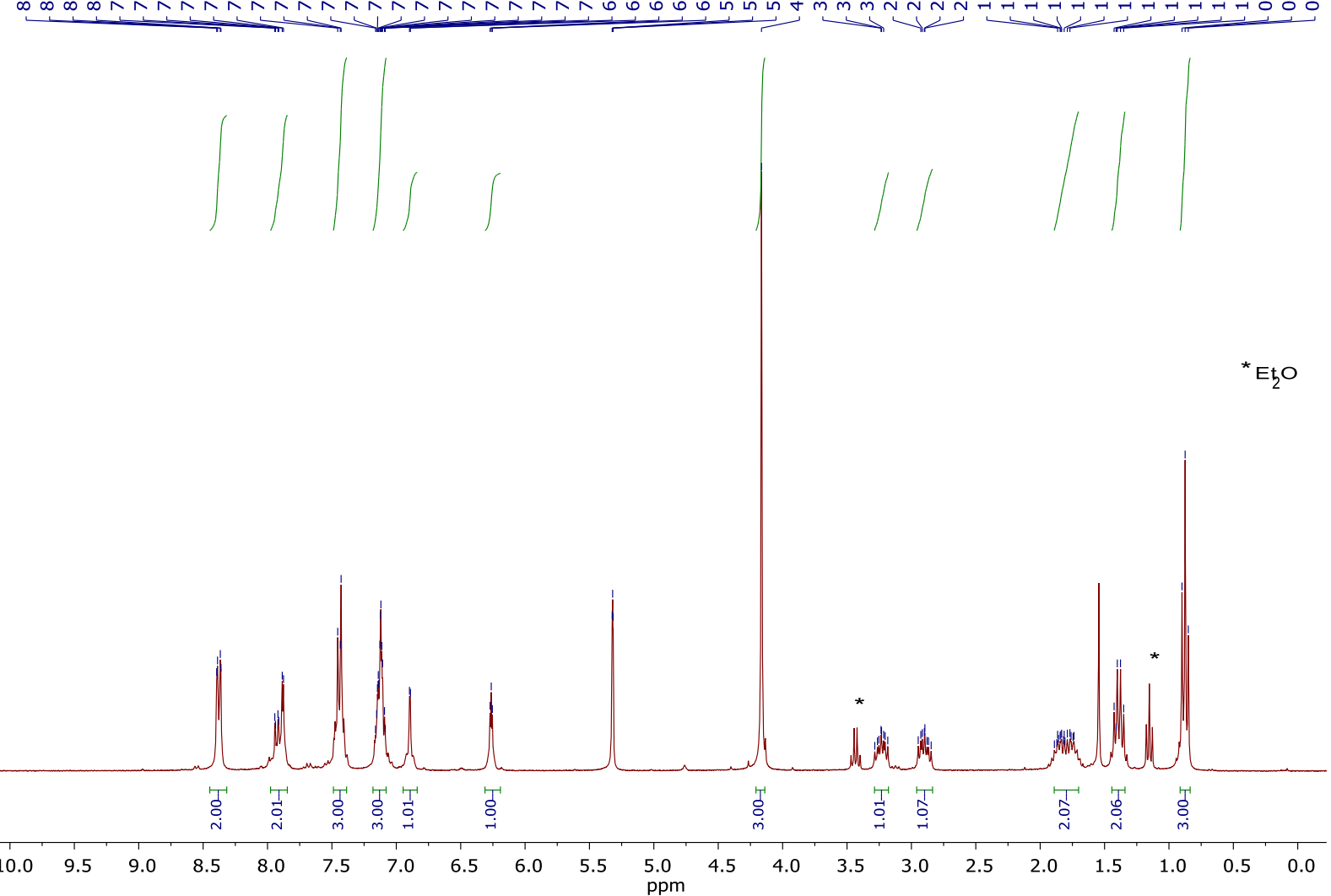

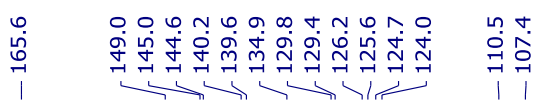

i্ji

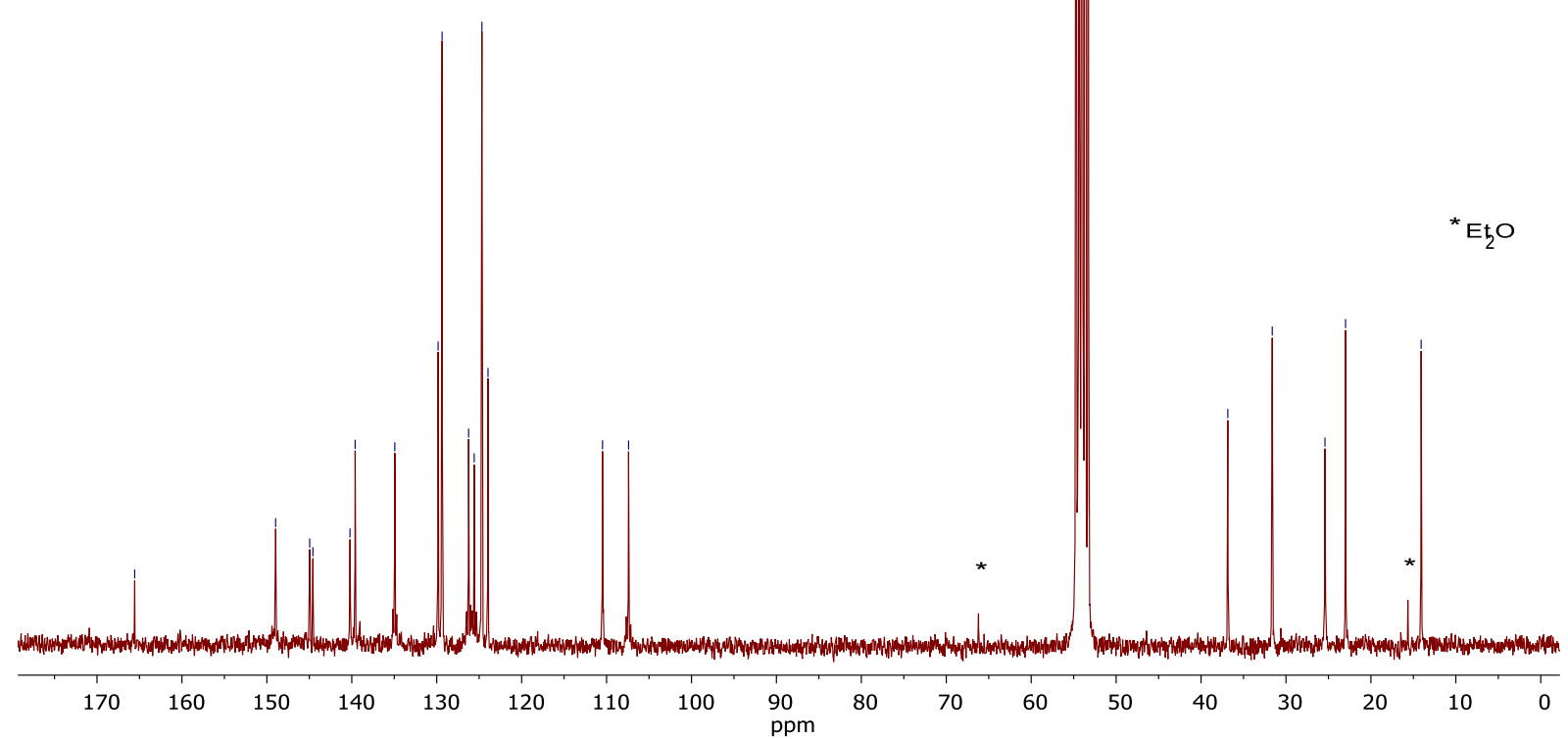

Figure S1. ${ }^{1} \mathrm{H}$ (top) and ${ }^{13} \mathrm{C}\left\{{ }^{1} \mathrm{H}\right\}$ APT (bottom) NMR spectra of complex trans-C, $C^{*}-\mathbf{2 a}\left(\mathrm{CD}_{2} \mathrm{Cl}_{2}, 300\right.$ and $75 \mathrm{MHz}$, respectively) 

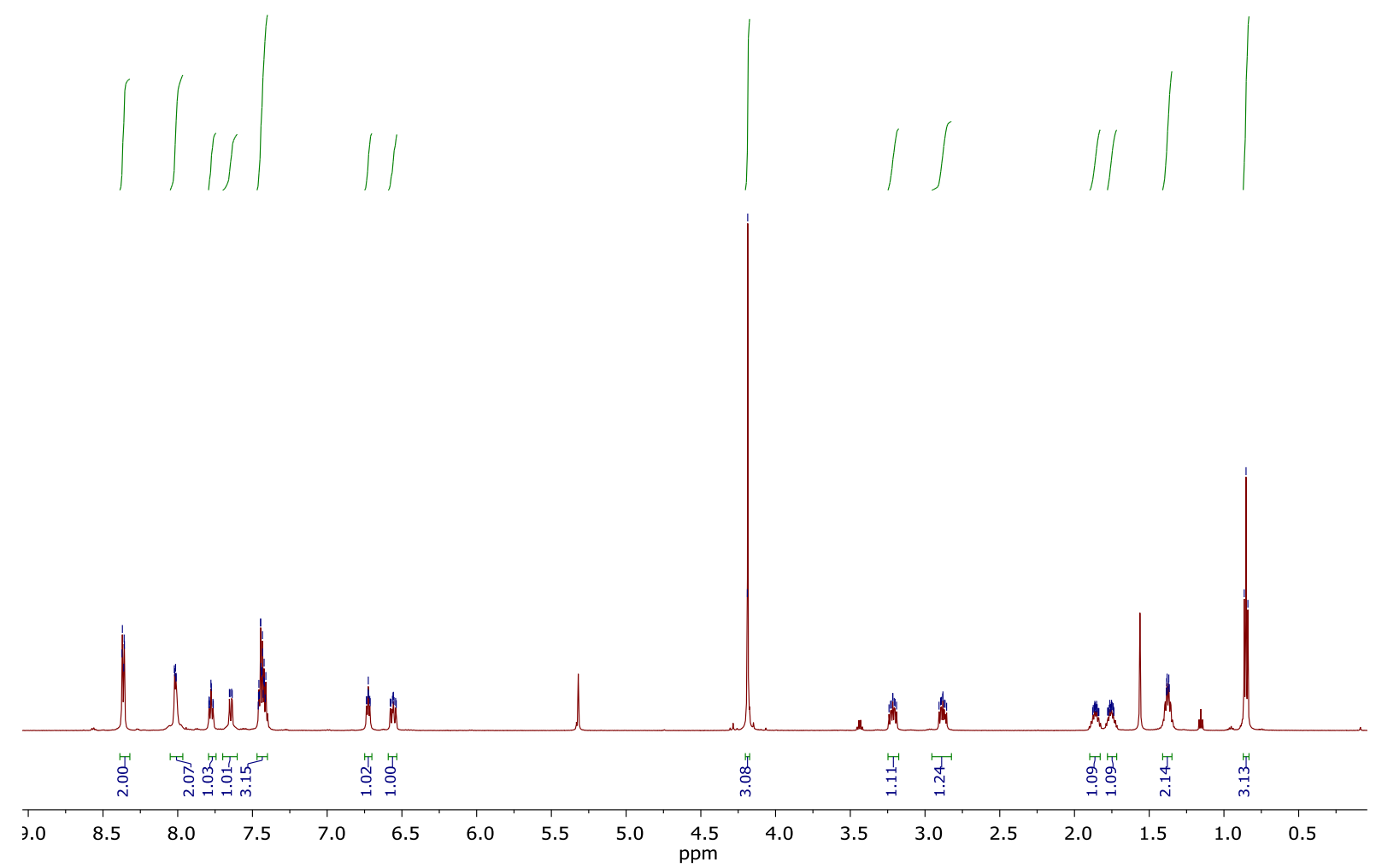

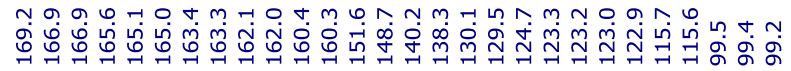

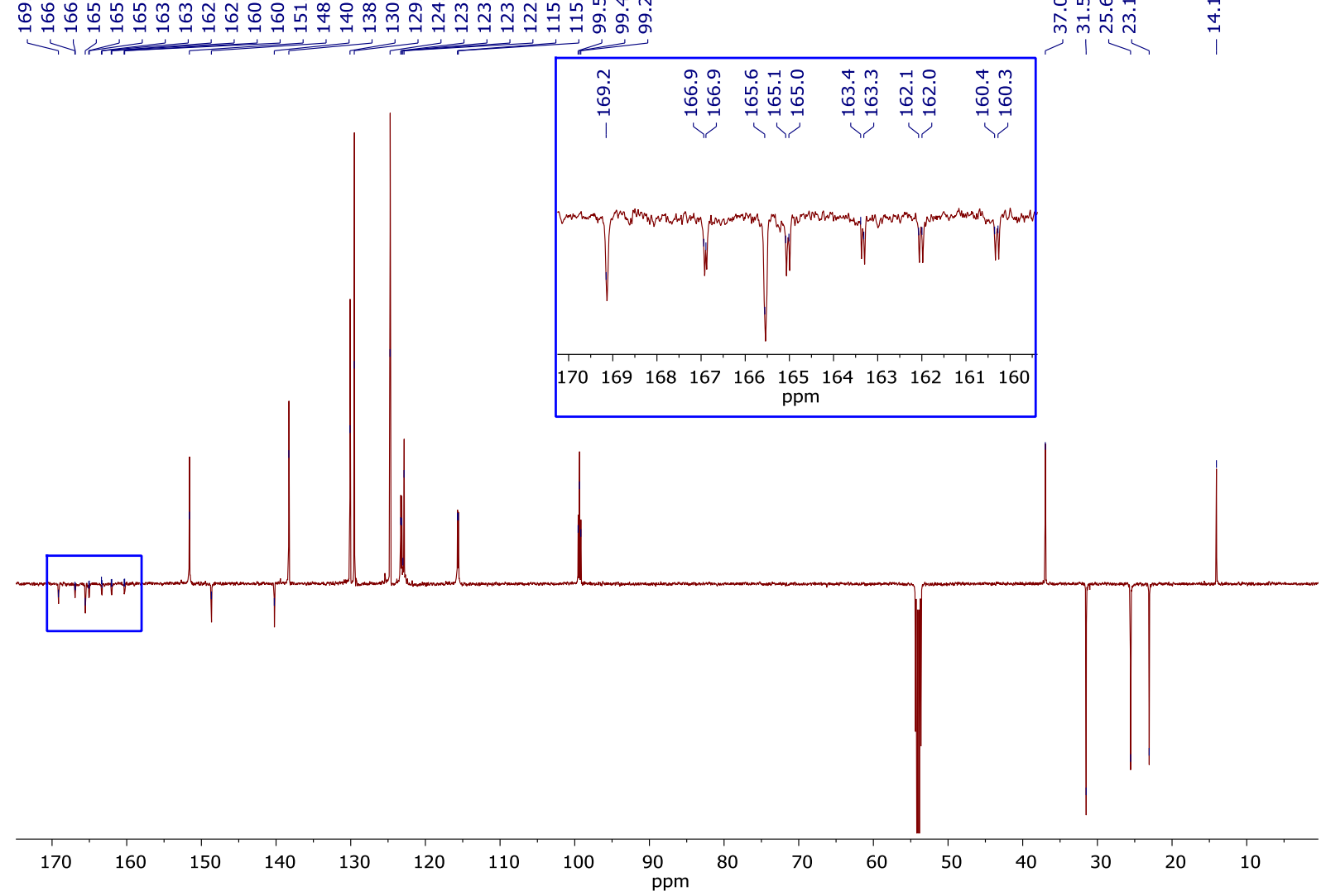

Figure S2. ${ }^{1} \mathrm{H}$ (top) and ${ }^{13} \mathrm{C}\left\{{ }^{1} \mathrm{H}\right\}$ APT (bottom) NMR spectra of complex trans-C, $C^{*}-\mathbf{2 b}\left(\mathrm{CD}_{2} \mathrm{Cl}_{2}, 600\right.$ and $151 \mathrm{MHz}$, respectively). 


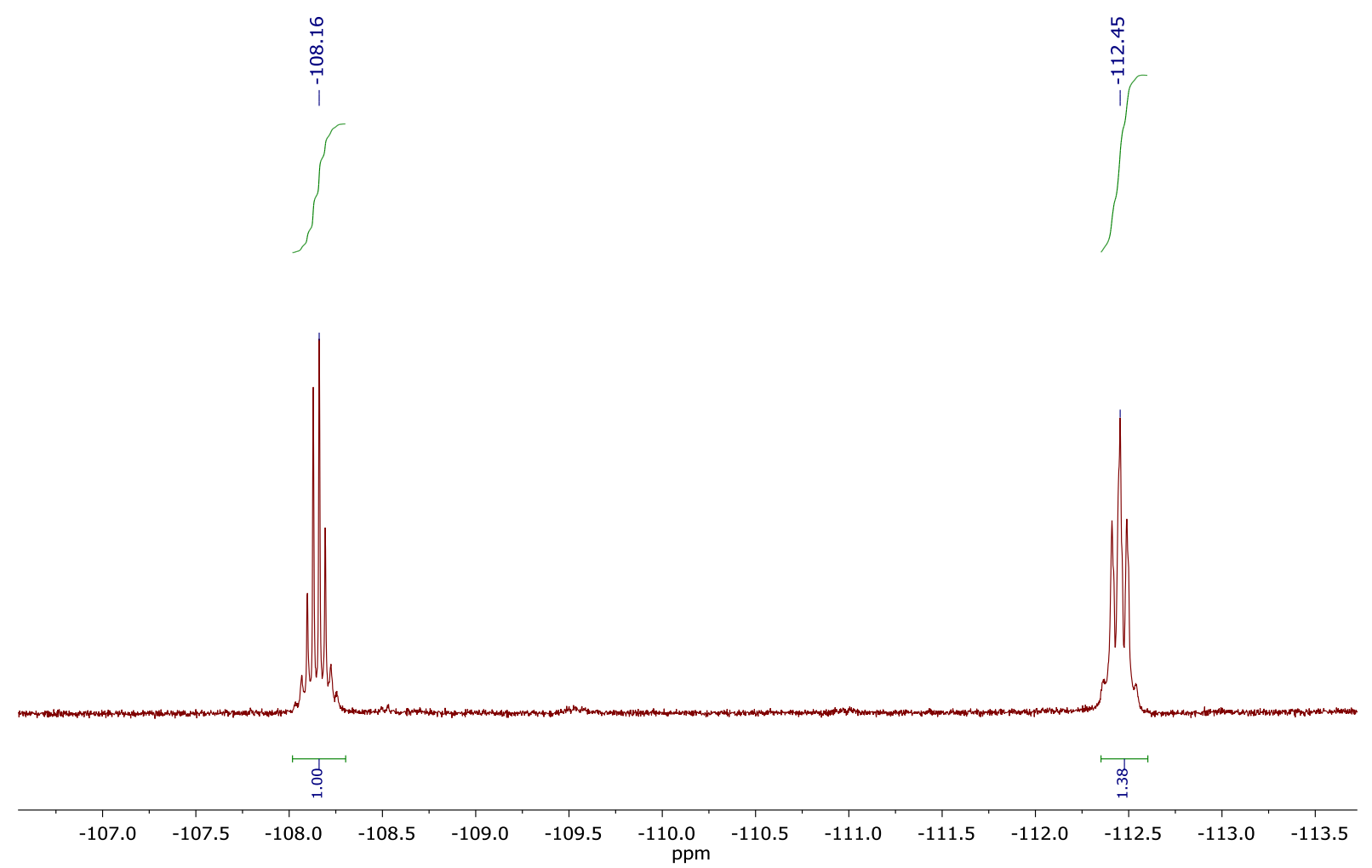

Figure S3. ${ }^{19} \mathrm{~F}$ NMR spectrum of complex trans-C, $C^{*}-\mathbf{2 b}\left(\mathrm{CD}_{2} \mathrm{Cl}_{2}, 282 \mathrm{MHz}\right)$. 


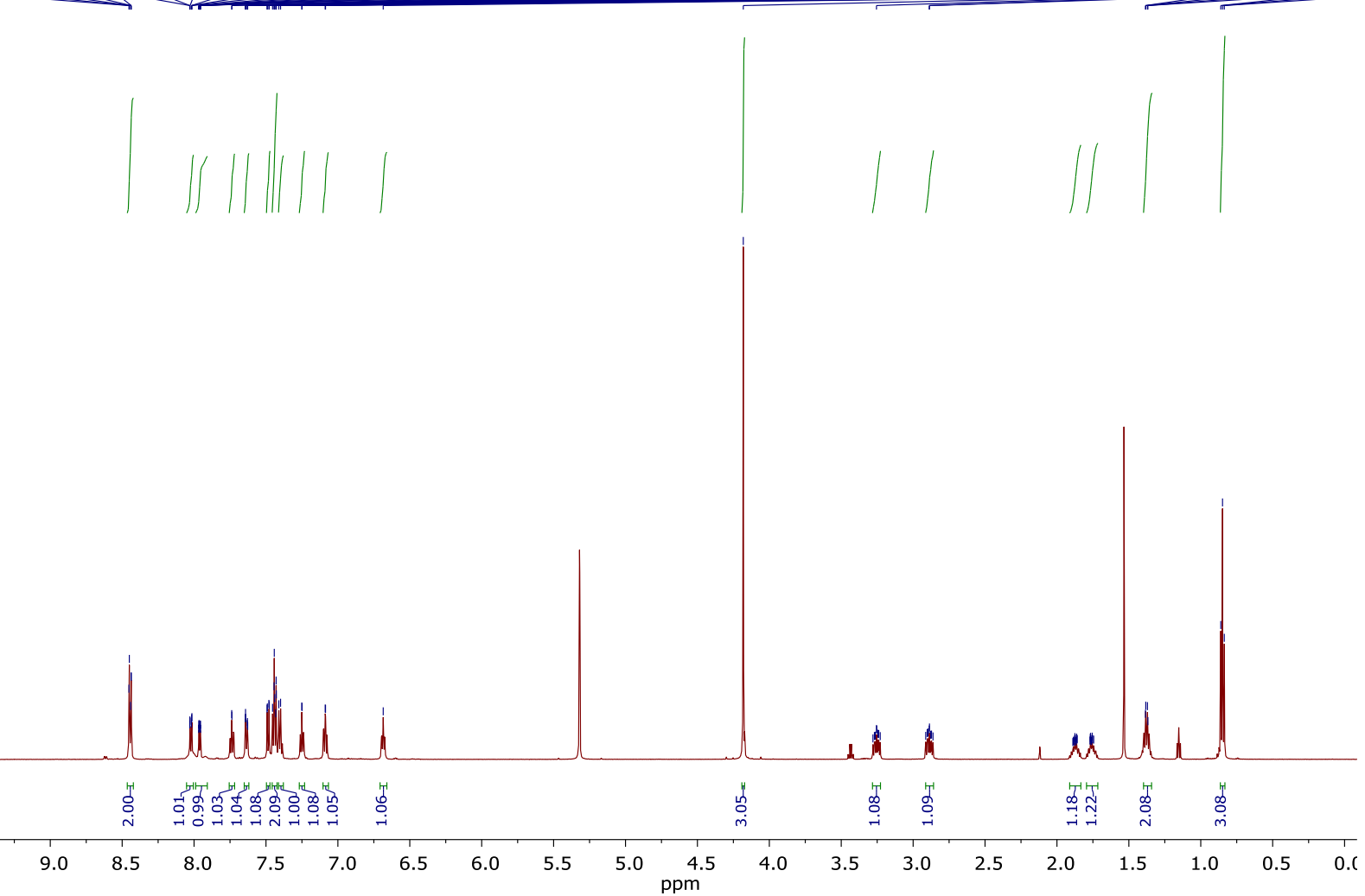

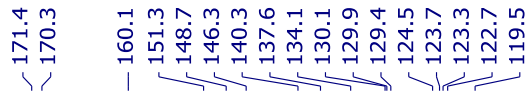

mìn

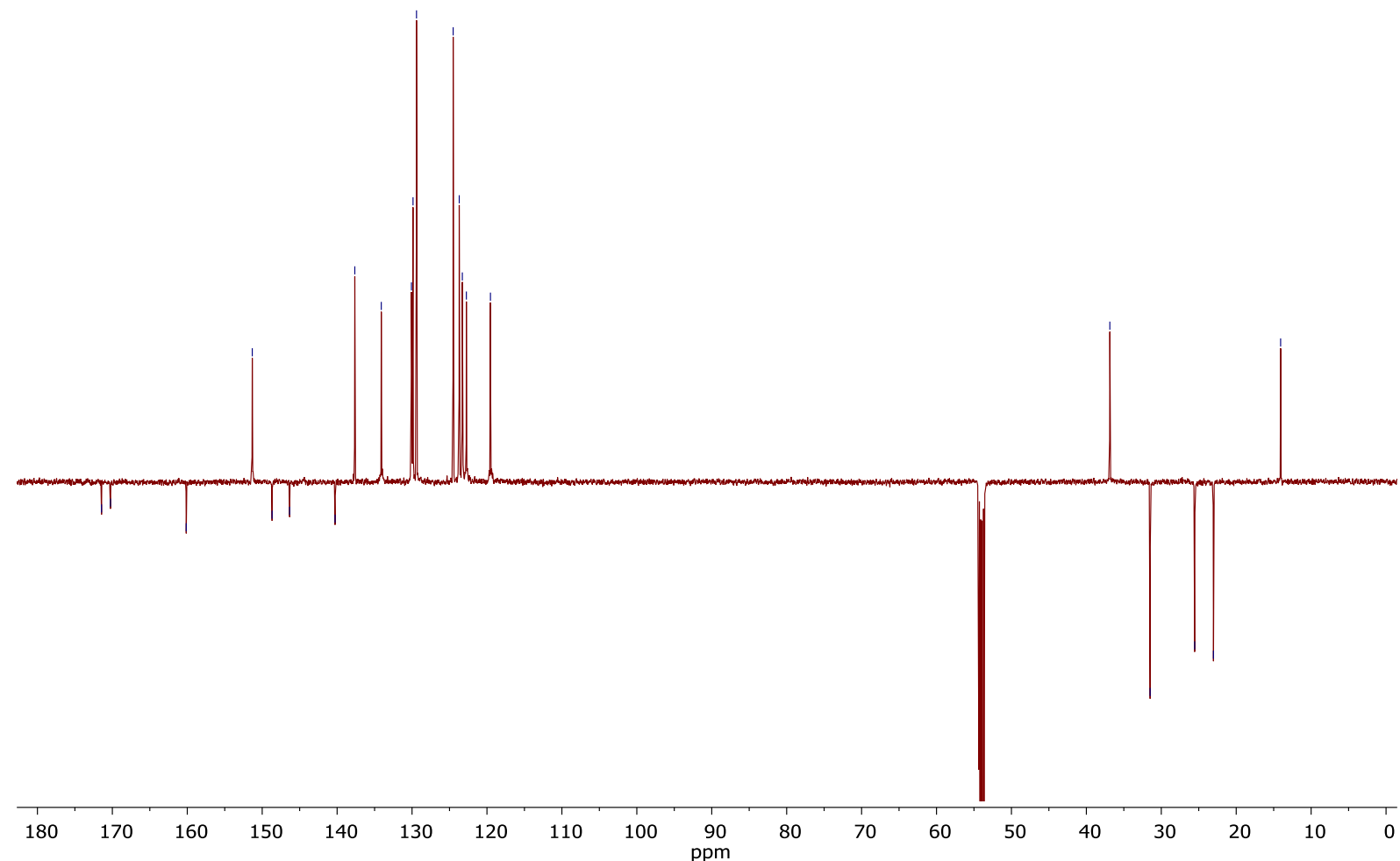

Figure S4. ${ }^{1} \mathrm{H}$ (top) and ${ }^{13} \mathrm{C}\left\{{ }^{1} \mathrm{H}\right\}$ APT (bottom) NMR spectra of complex trans-C, $C^{*}-\mathbf{2 c}\left(\mathrm{CD}_{2} \mathrm{Cl}_{2}, 600\right.$ and $151 \mathrm{MHz}$, respectively). 

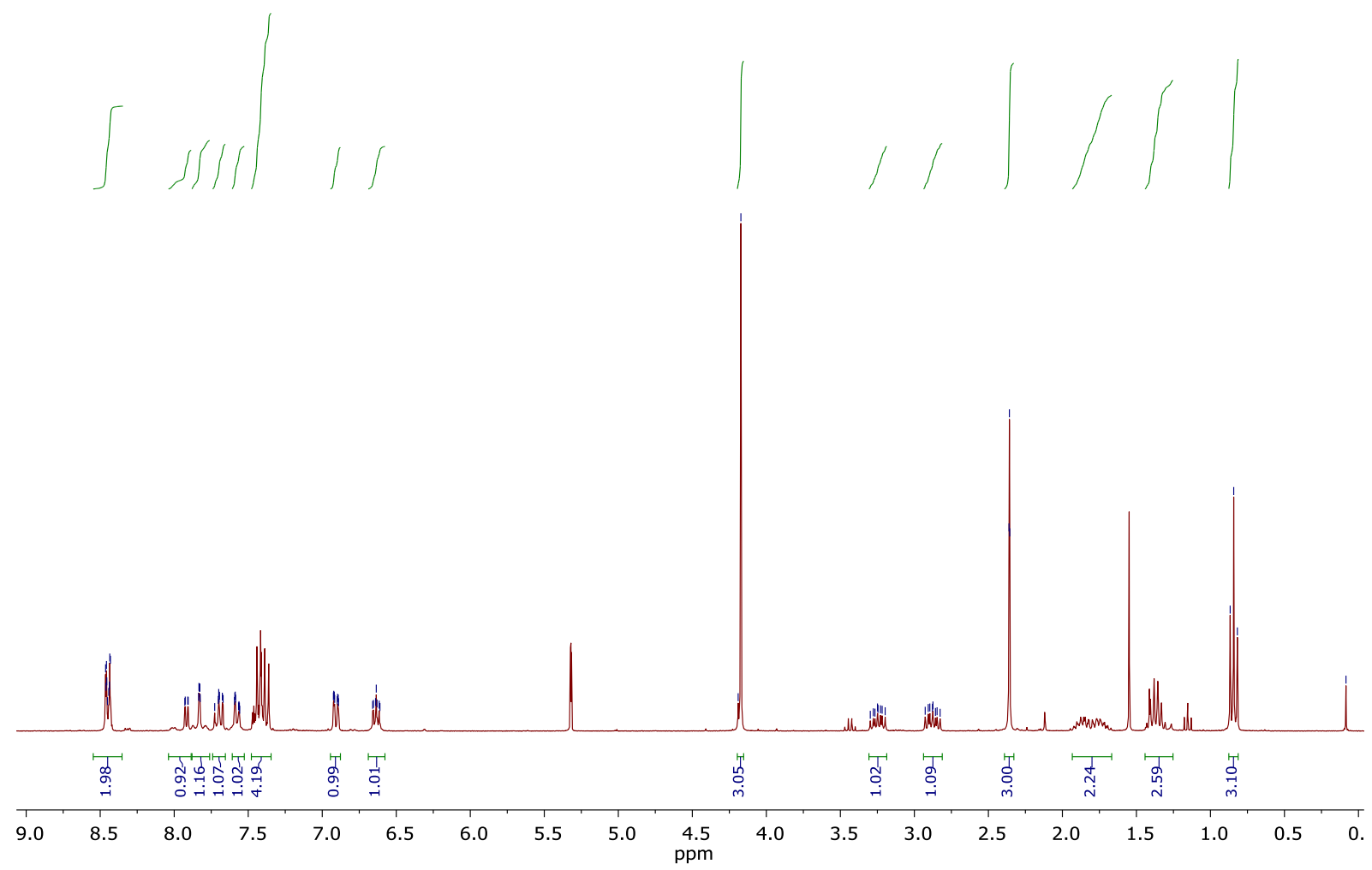

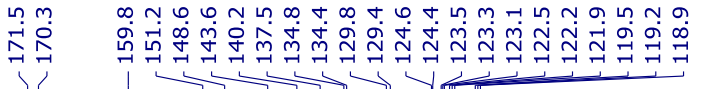

îj

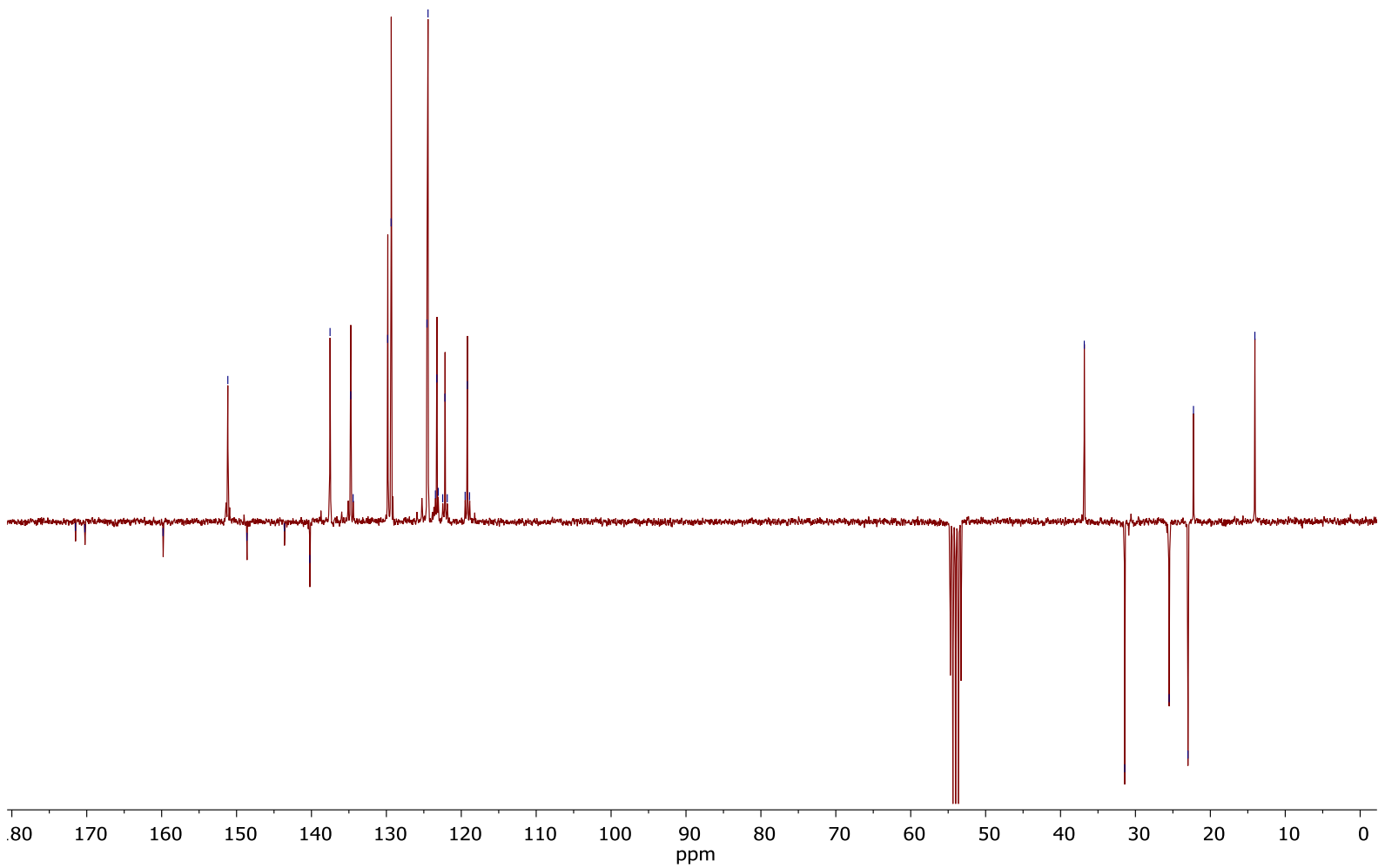

Figure S5. ${ }^{1} \mathrm{H}$ (top) and ${ }^{13} \mathrm{C}\left\{{ }^{1} \mathrm{H}\right\}$ APT (bottom) NMR spectra of complex trans-C, $C^{*}-\mathbf{2 d}\left(\mathrm{CD}_{2} \mathrm{Cl}_{2}, 300\right.$ and $75 \mathrm{MHz}$, respectively). 

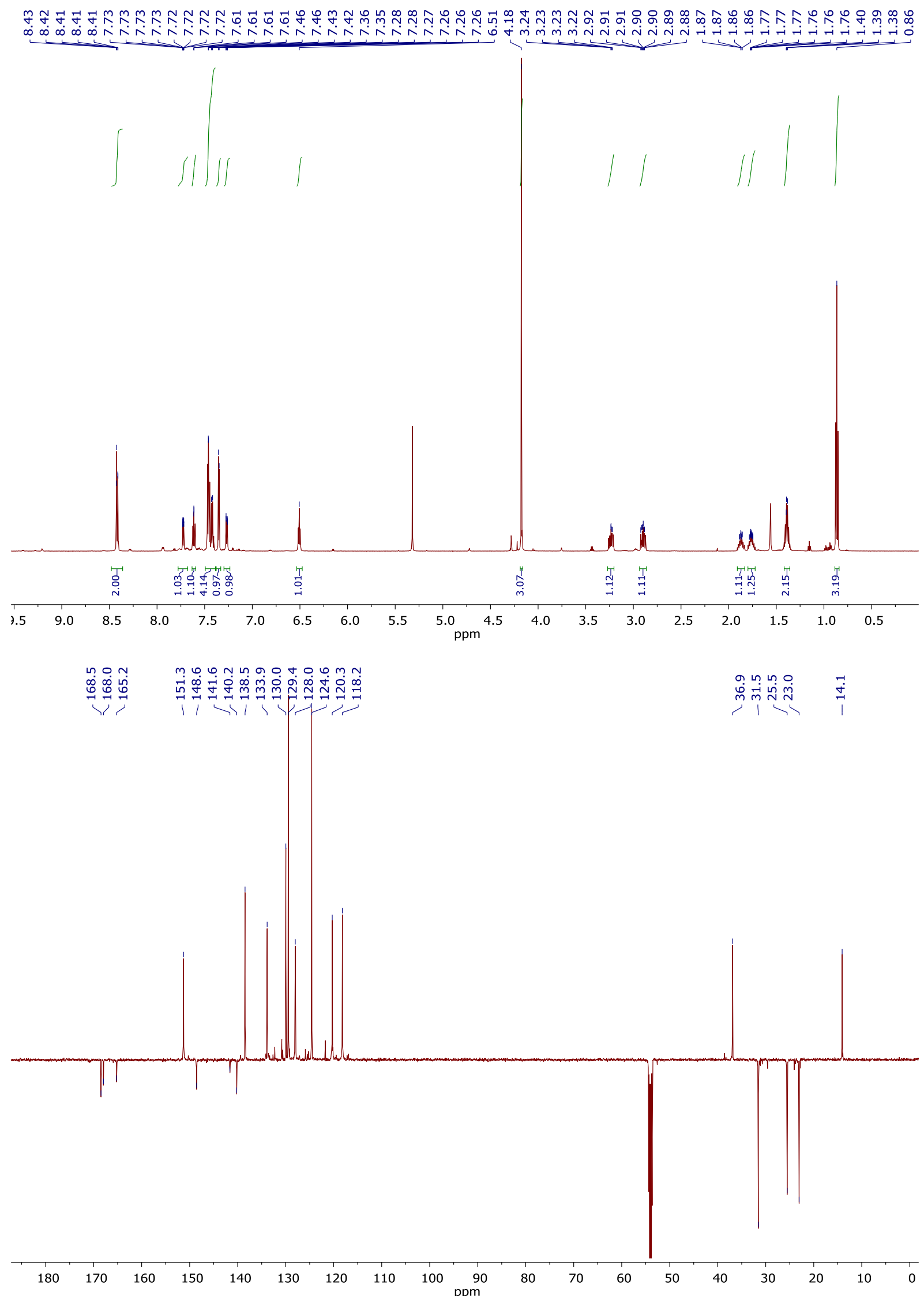

Figure S6. ${ }^{1} \mathrm{H}$ (top) and ${ }^{13} \mathrm{C}\left\{{ }^{1} \mathrm{H}\right\}$ APT (bottom) NMR spectra of complex trans-C, $C^{*}-2 \mathrm{e}\left(\mathrm{CD}_{2} \mathrm{Cl}_{2}, 600\right.$ and $151 \mathrm{MHz}$, respectively). 

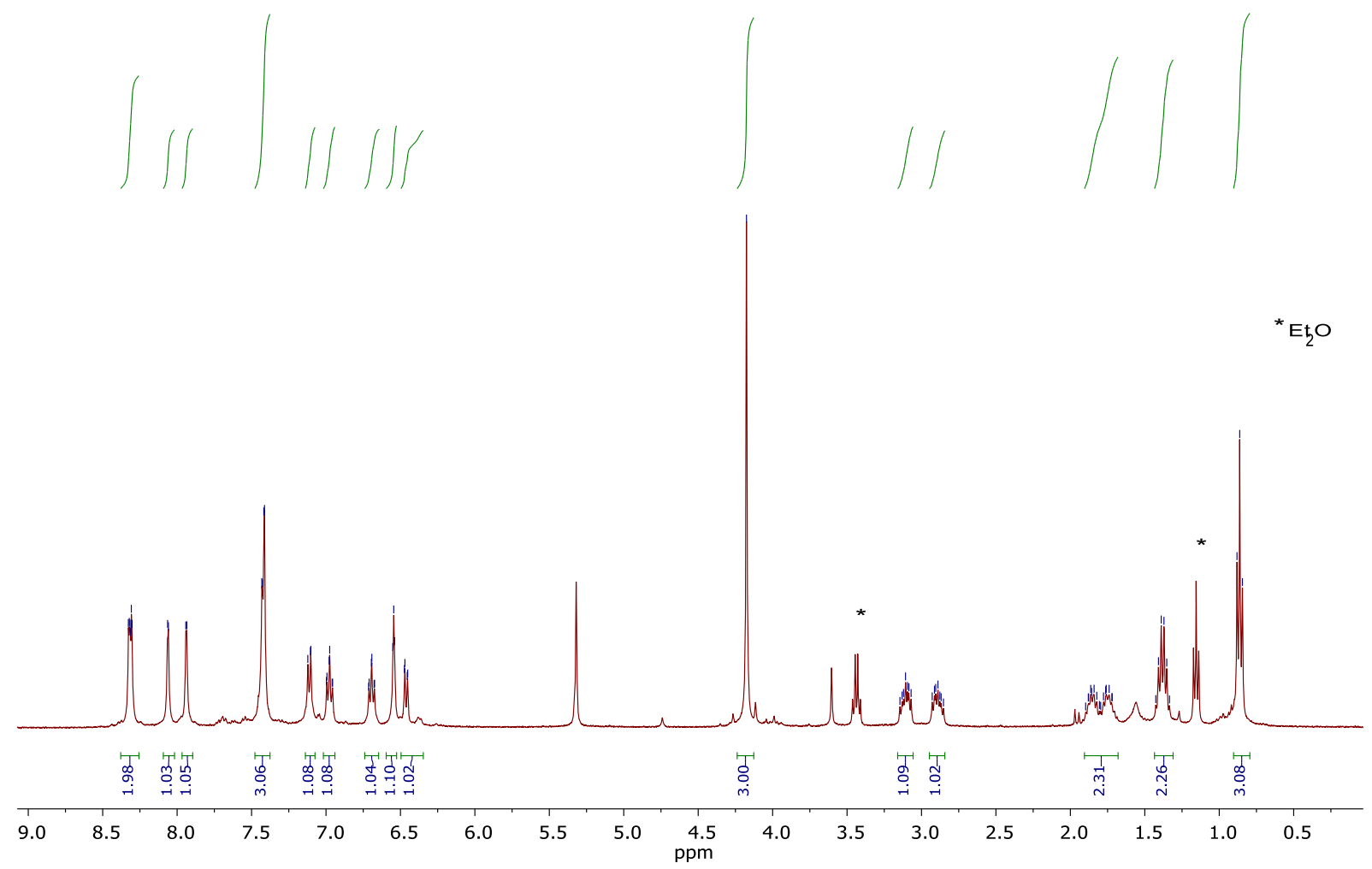

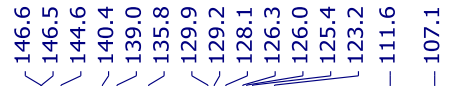

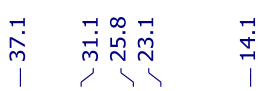

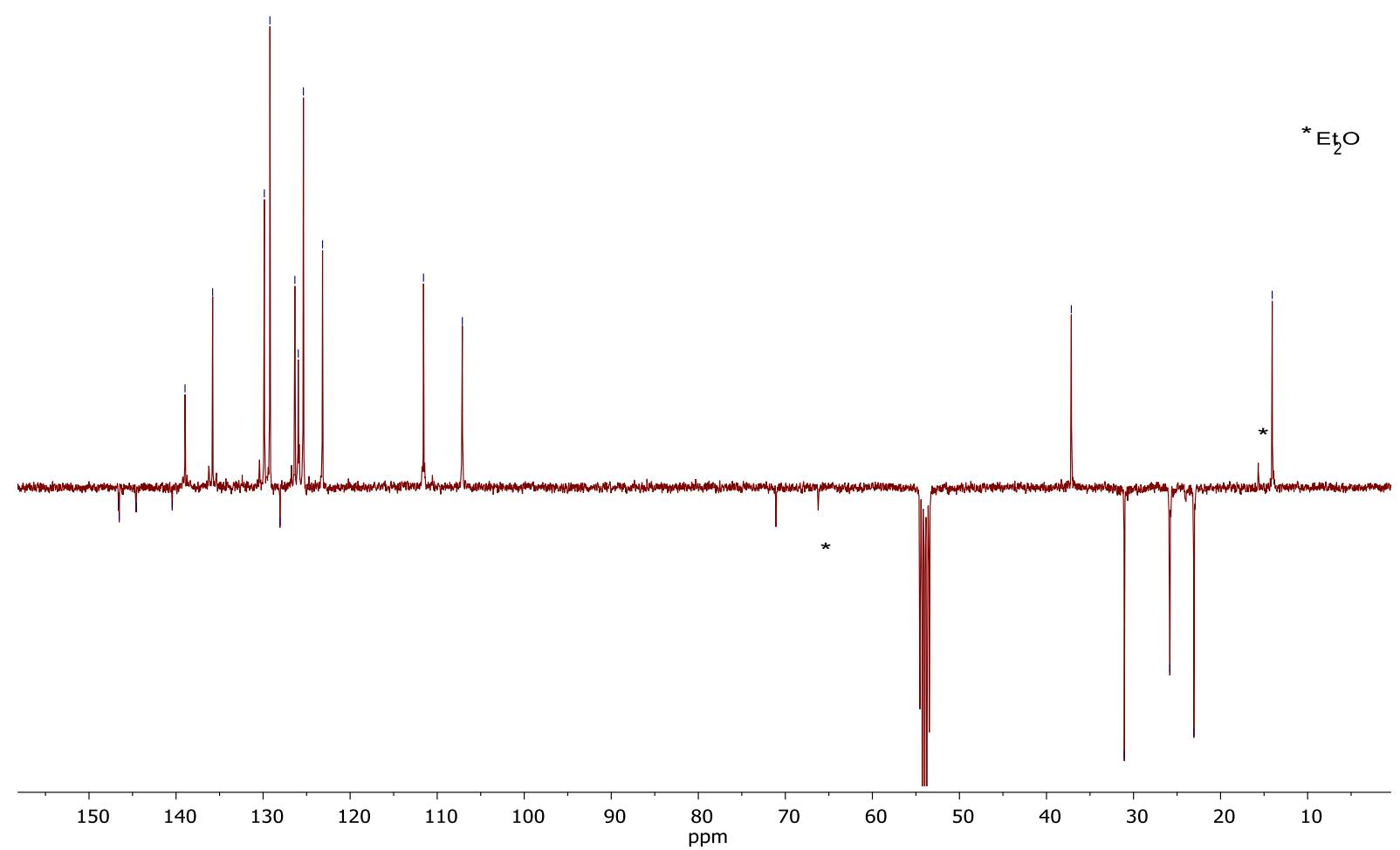

Figure S7. ${ }^{1} \mathrm{H}$ (top) and ${ }^{13} \mathrm{C}\left\{{ }^{1} \mathrm{H}\right\}$ APT (bottom) NMR spectra of complex cis-C, $C^{*}-2 a\left(\mathrm{CD}_{2} \mathrm{Cl}_{2}, 400\right.$ and $100 \mathrm{MHz}$, respectively). 


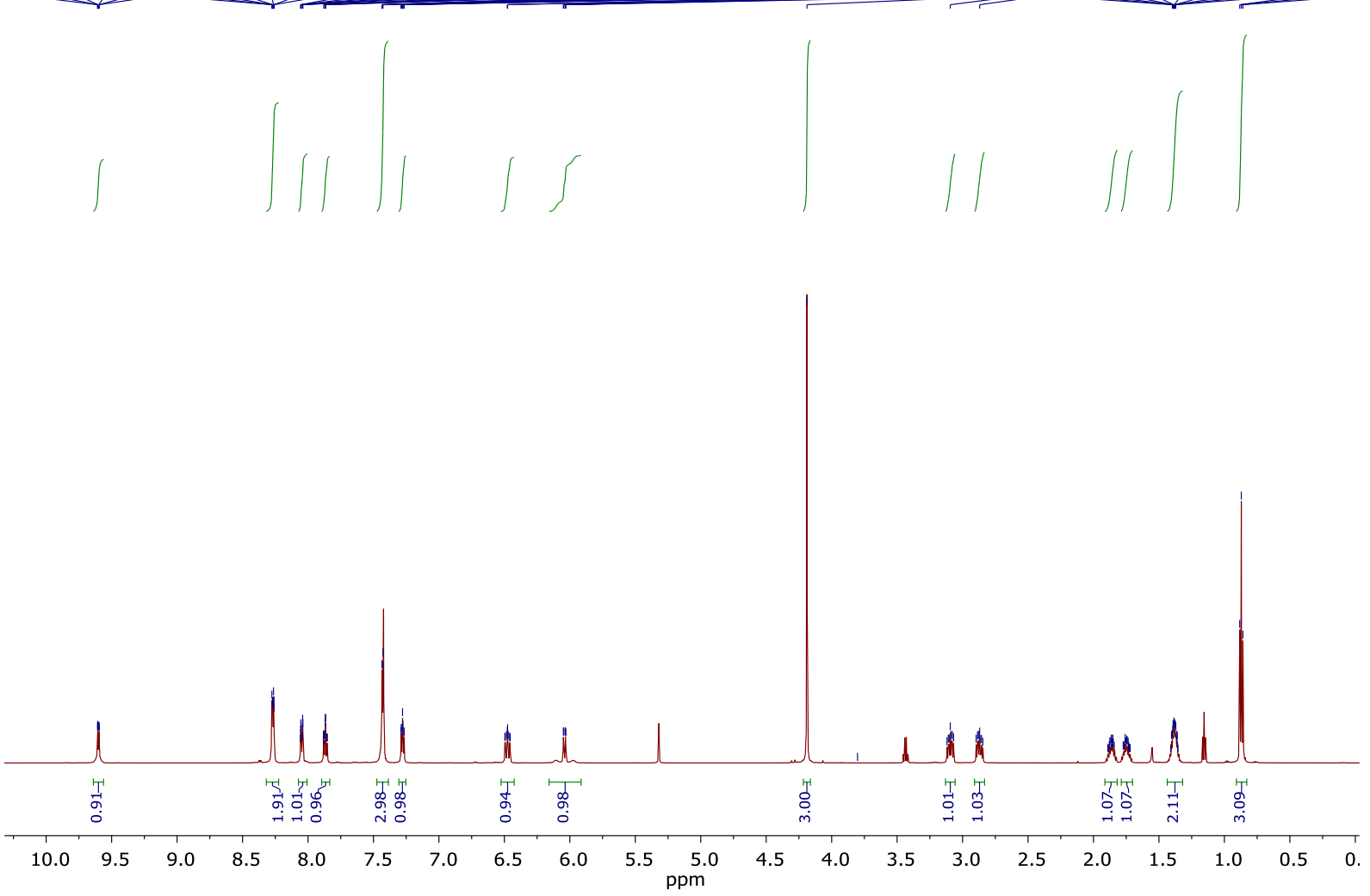

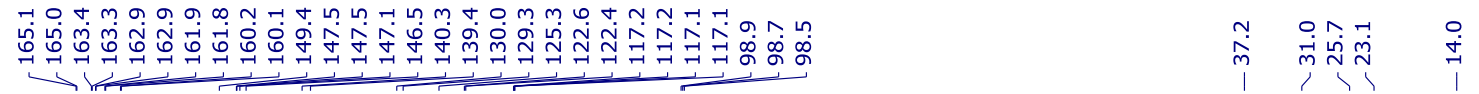

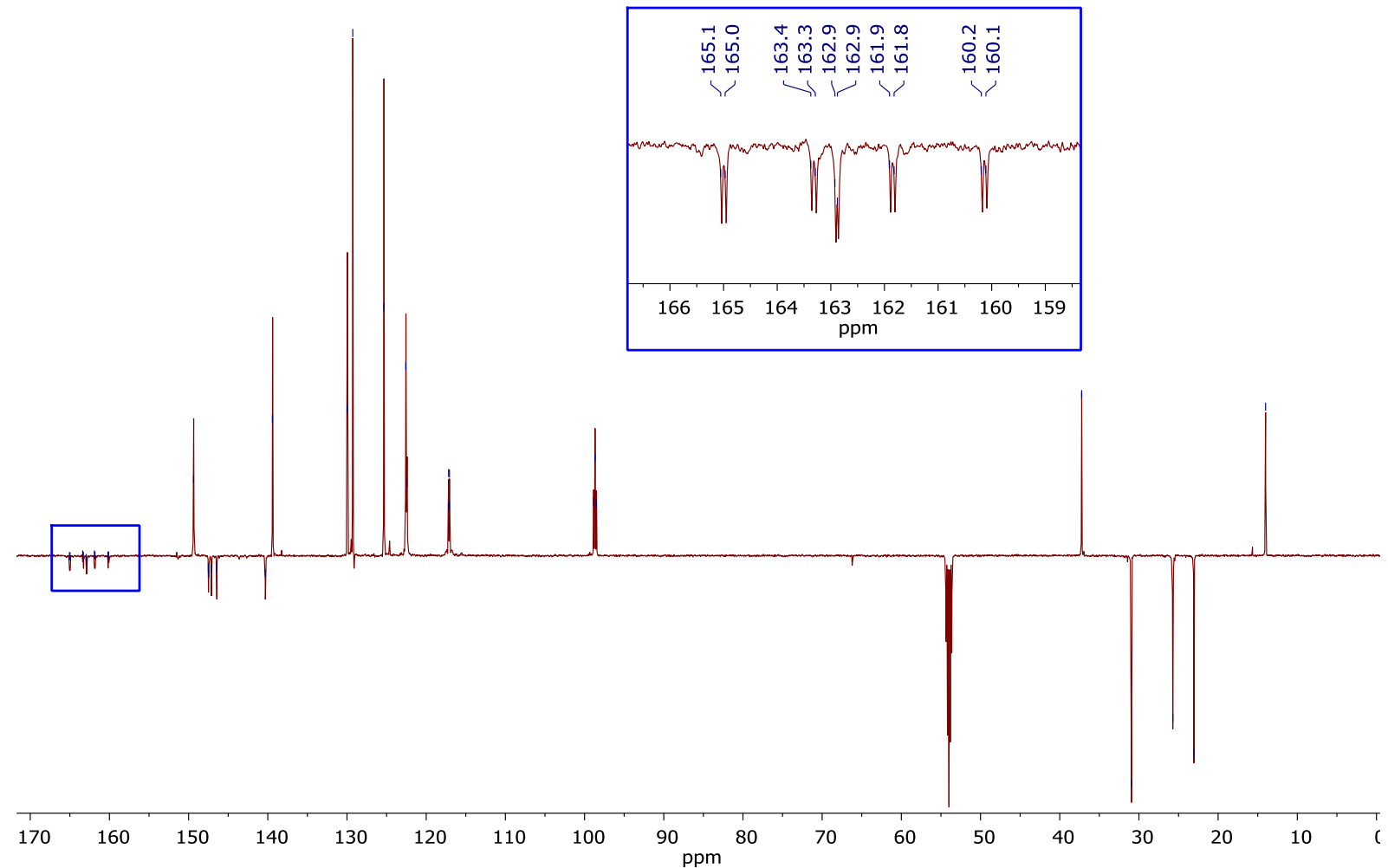

Figure S8. ${ }^{1} \mathrm{H}$ (top) and ${ }^{13} \mathrm{C}\left\{{ }^{1} \mathrm{H}\right\}$ APT (bottom) NMR spectra of complex cis-C, $C^{*}-\mathbf{2 b} \quad\left(\mathrm{CD}_{2} \mathrm{Cl}_{2}, 600\right.$ and $151 \mathrm{MHz}$, respectively). 


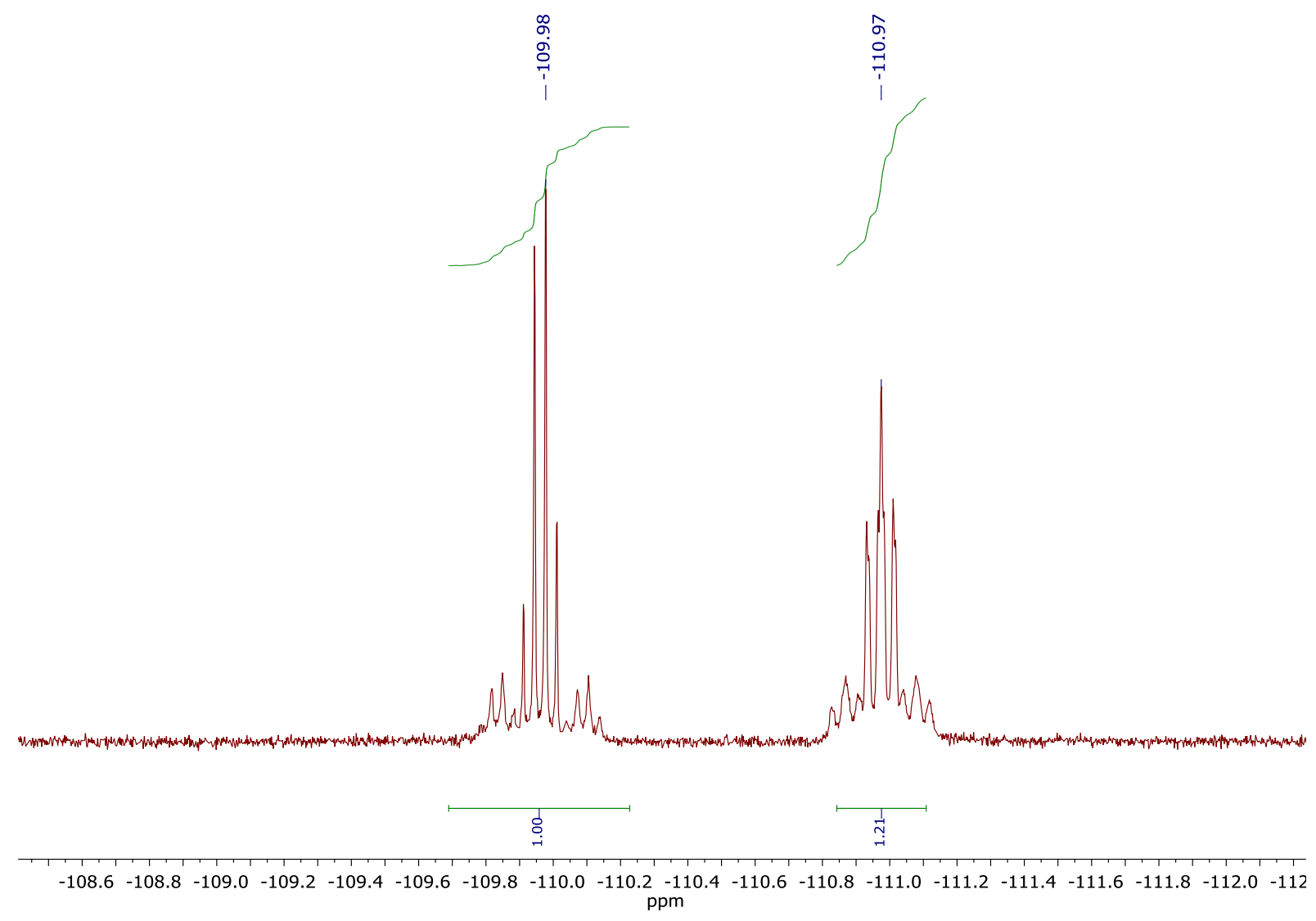

Figure S9. ${ }^{19} \mathrm{~F}$ NMR spectrum of complex cis-C,C $C_{-}^{*} \mathbf{2 b}\left(\mathrm{CD}_{2} \mathrm{Cl}_{2}, 282 \mathrm{MHz}\right)$. 


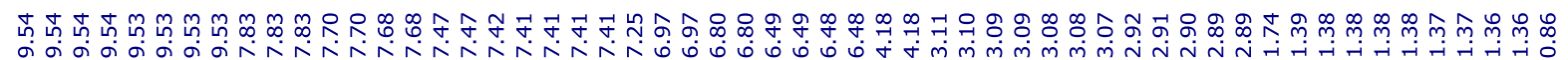

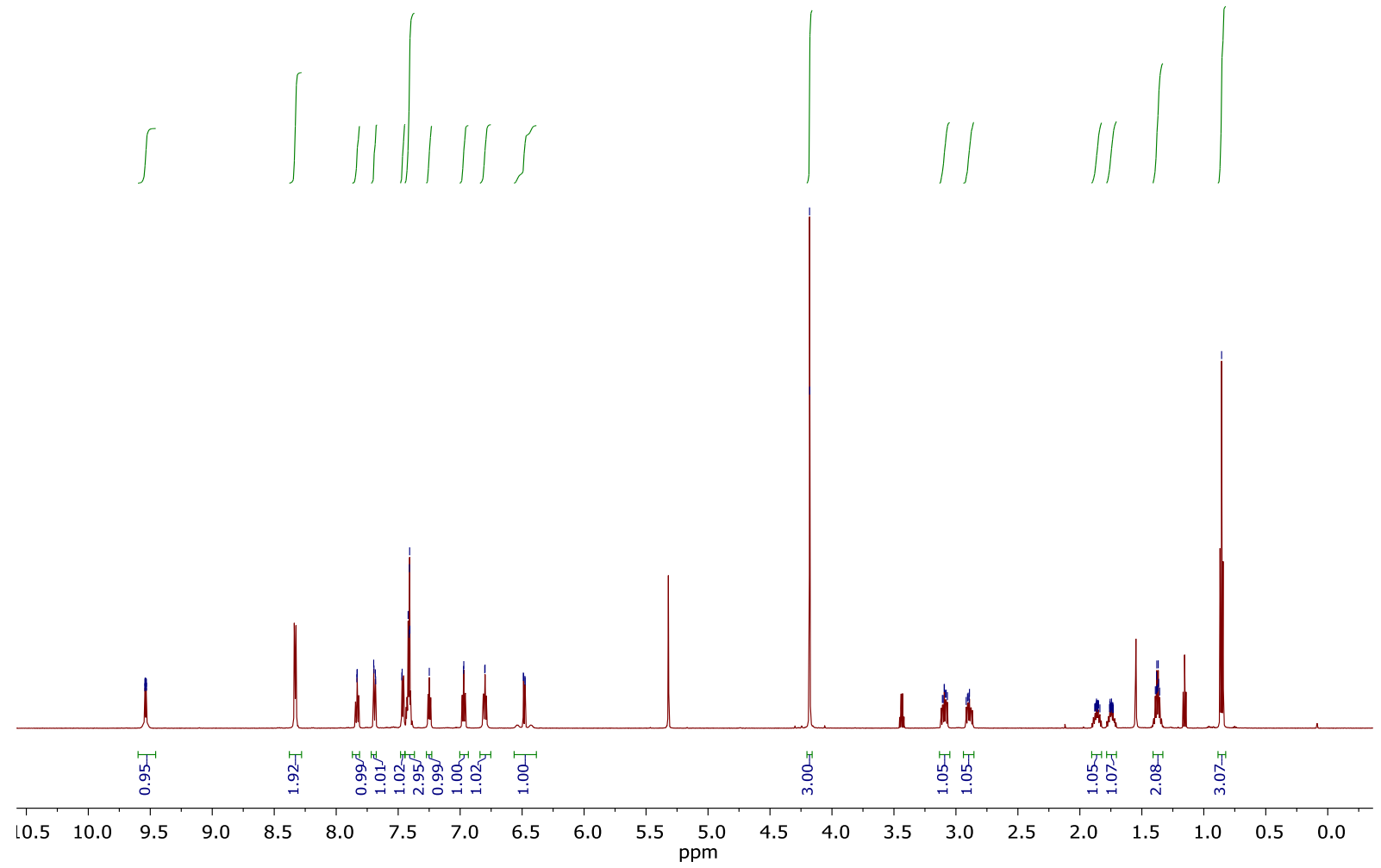

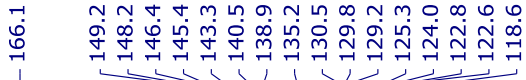

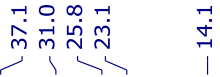

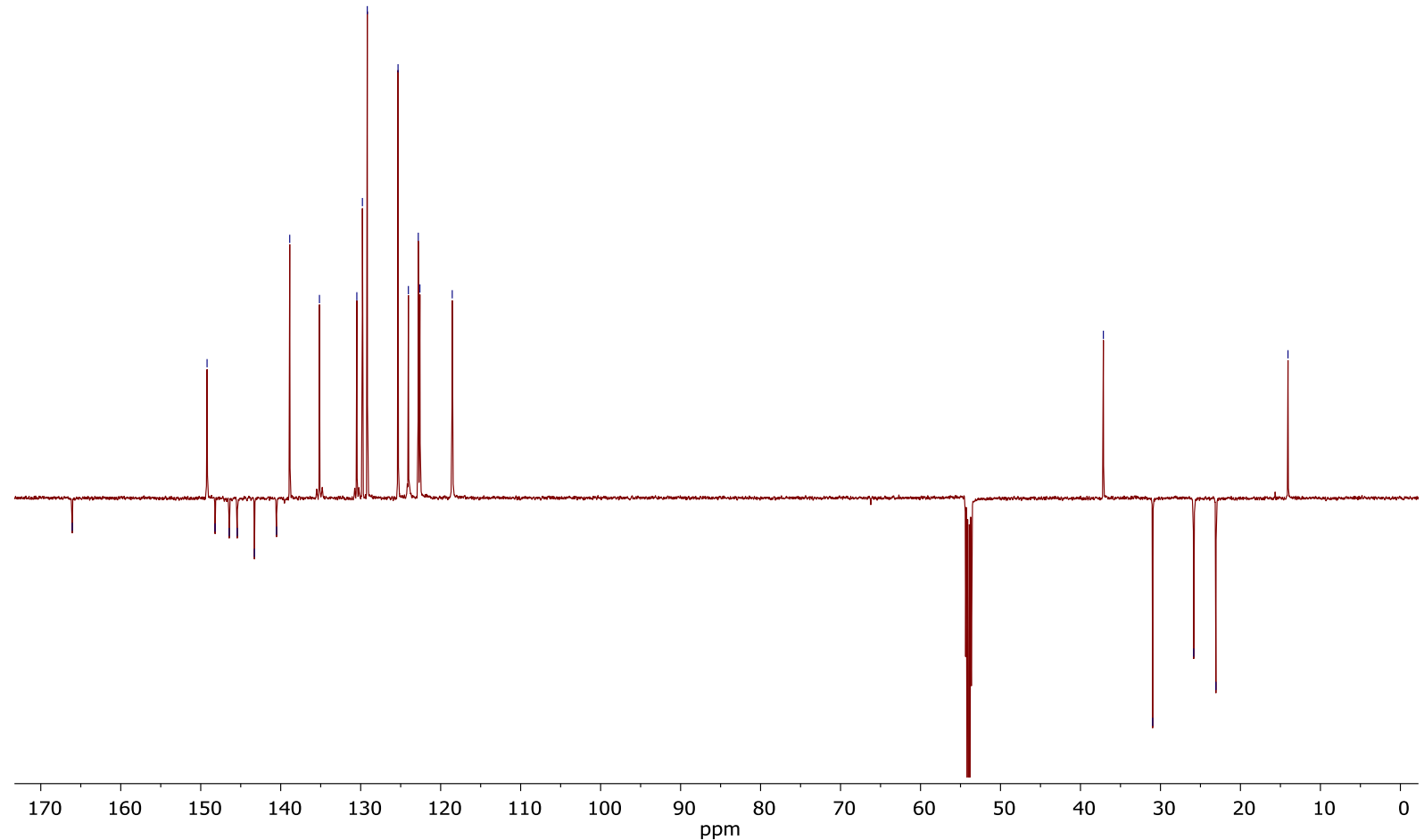

Figure S10. ${ }^{1} \mathrm{H}$ (top) and ${ }^{13} \mathrm{C}\left\{{ }^{1} \mathrm{H}\right\}$ APT (bottom) NMR spectra of complex cis-C, $C^{*}-\mathbf{2 c}\left(\mathrm{CD}_{2} \mathrm{Cl}_{2}, 600\right.$ and $151 \mathrm{MHz}$, respectively). 


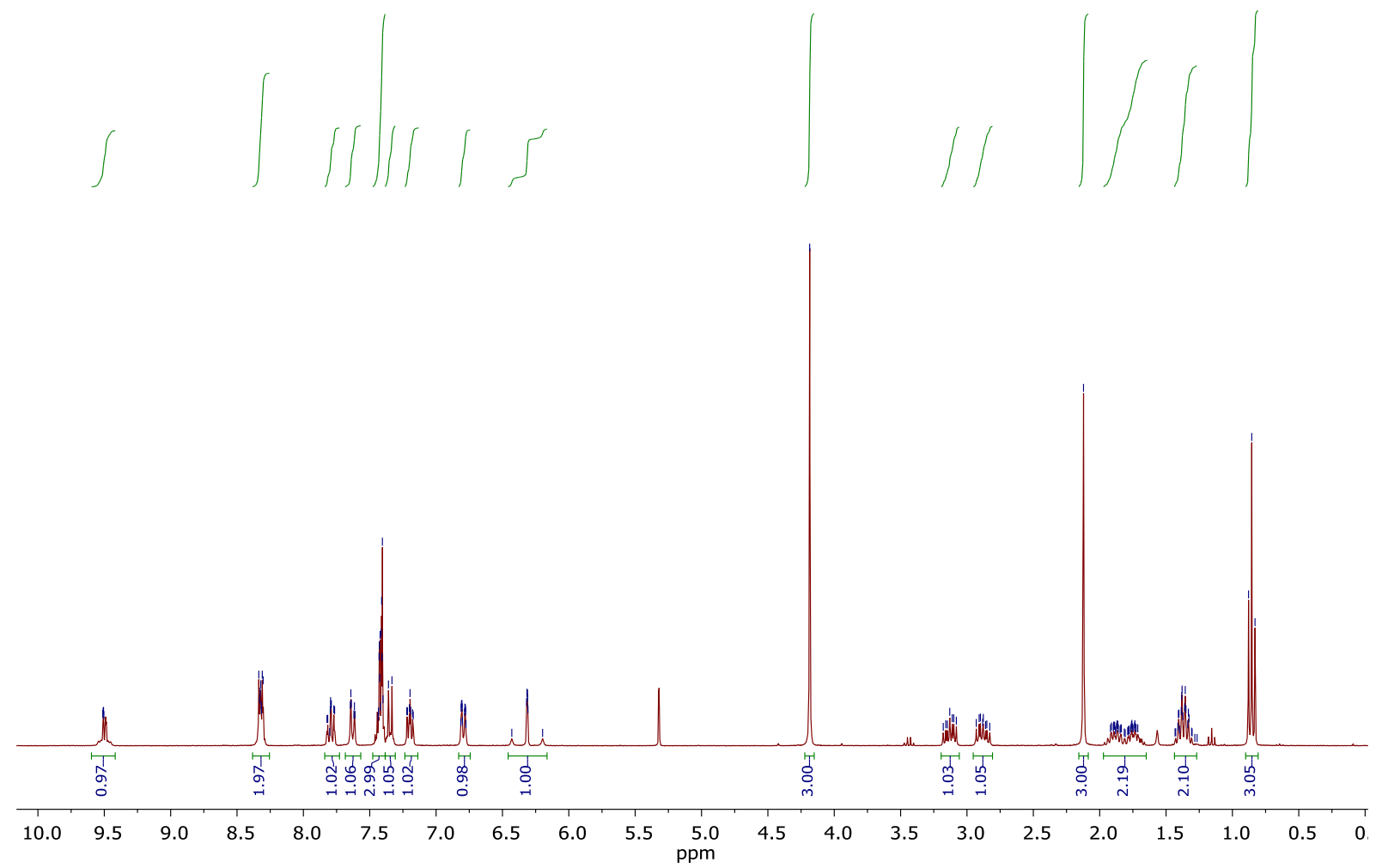

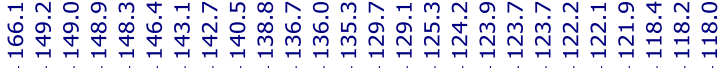

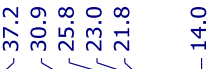

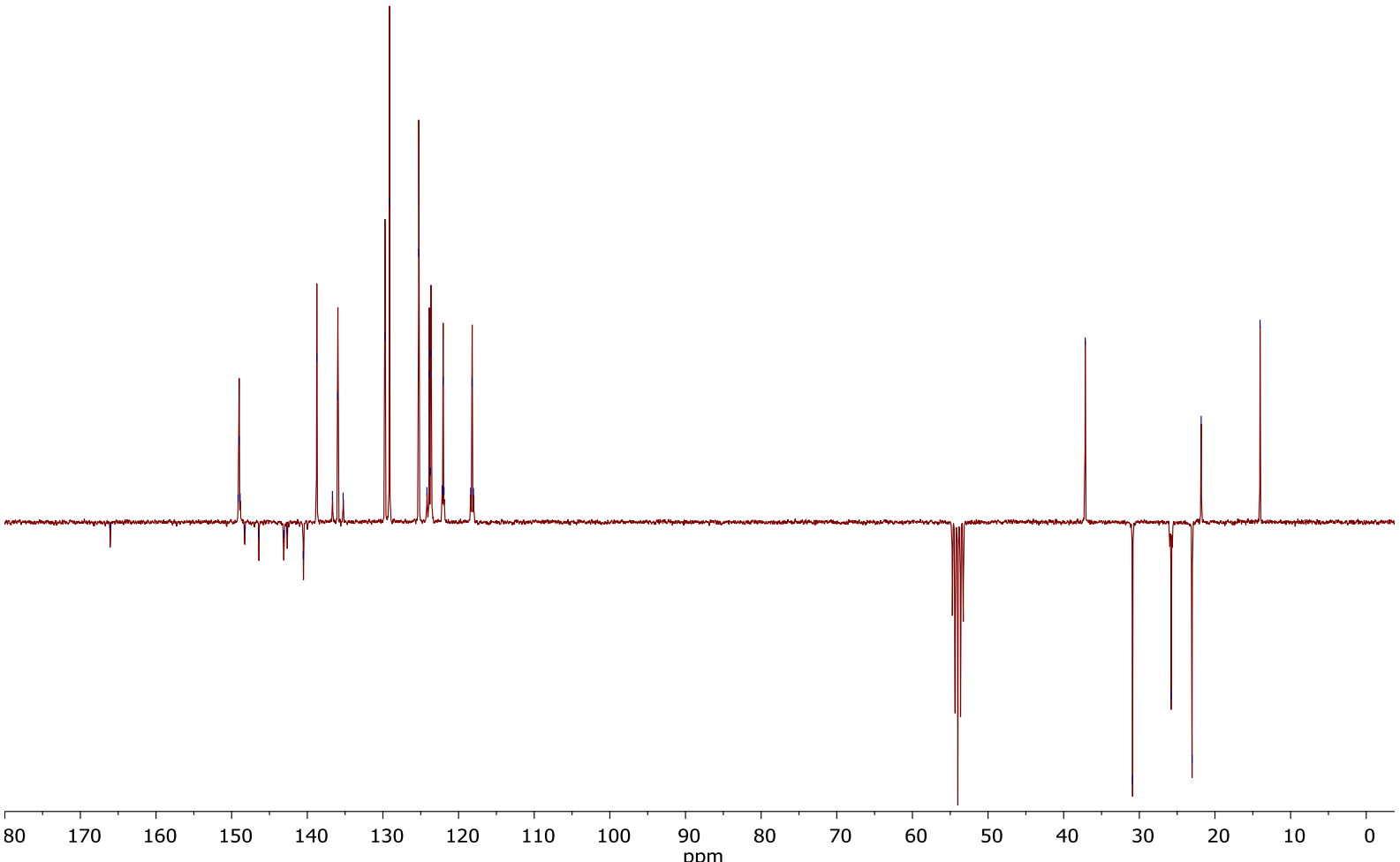

Figure S11. ${ }^{1} \mathrm{H}$ (top) and ${ }^{13} \mathrm{C}\left\{{ }^{1} \mathrm{H}\right\}$ APT (bottom) NMR spectra of complex cis-C, $C^{*-2 d}\left(\mathrm{CD}_{2} \mathrm{Cl}_{2}, 300\right.$ and $75 \mathrm{MHz}$, respectively). 


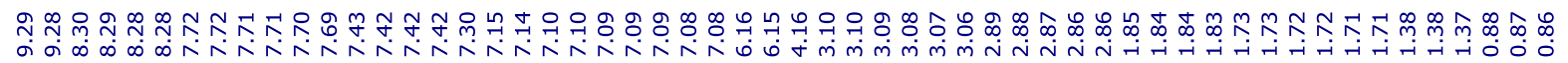
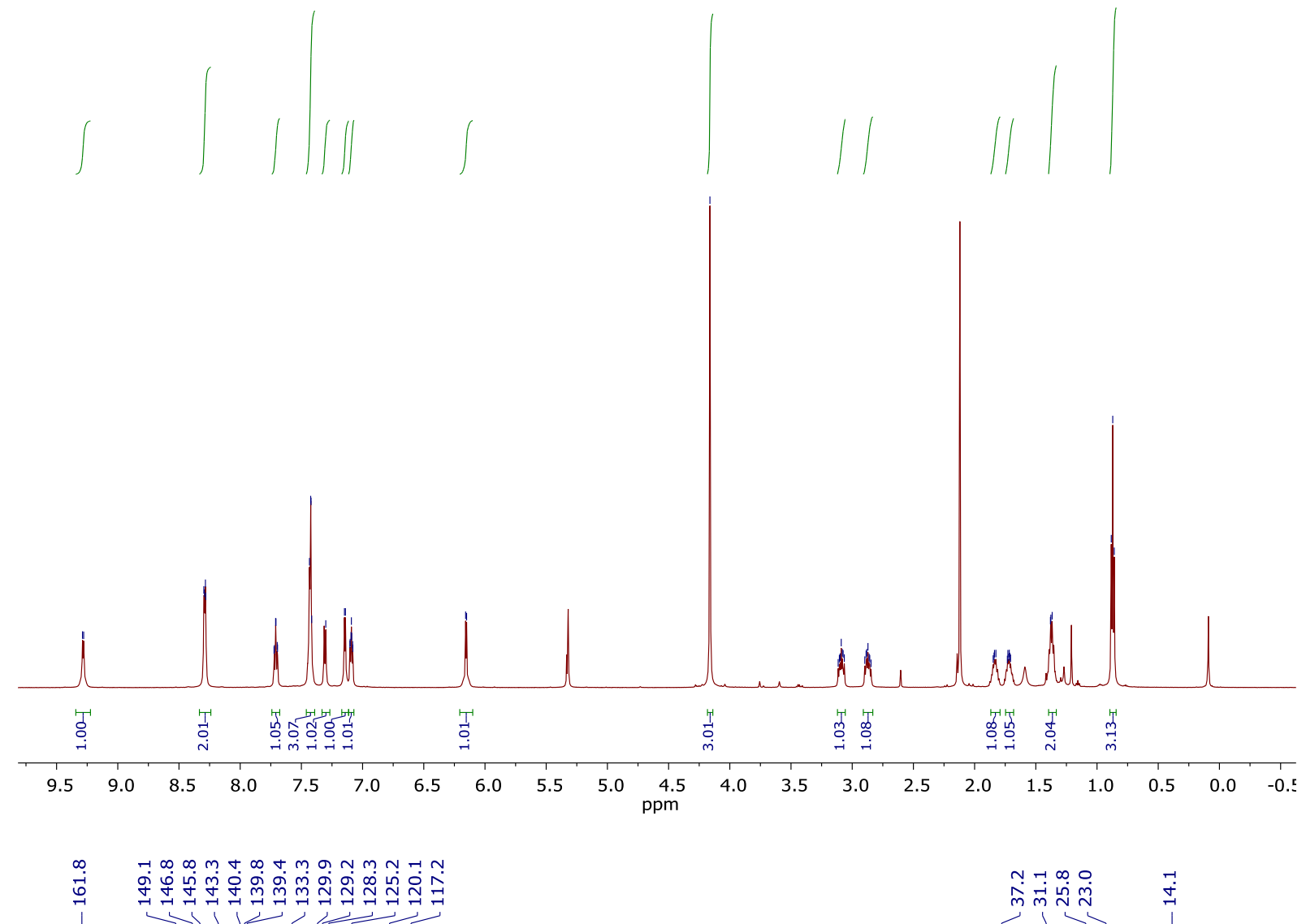

華

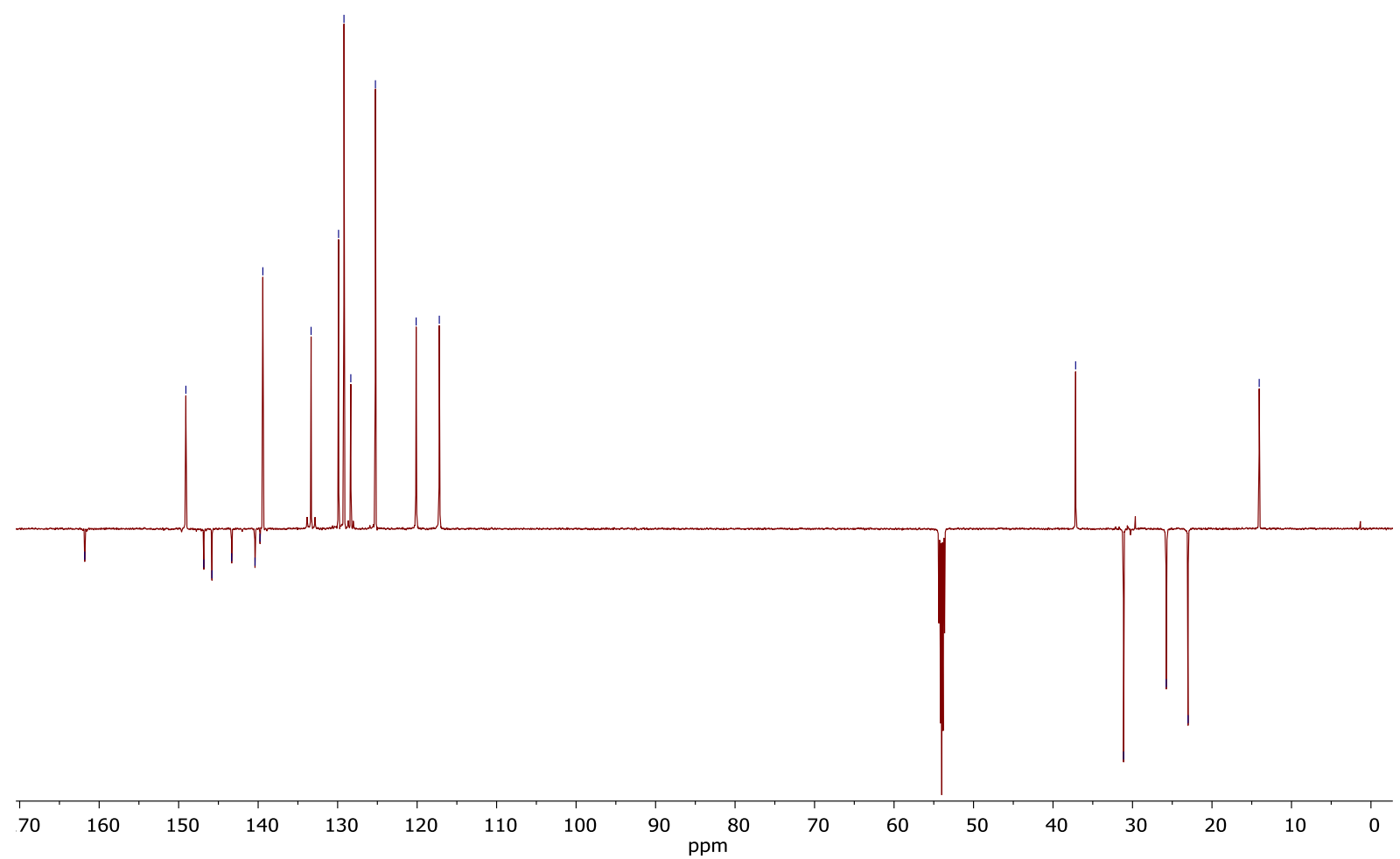

Figure S12. ${ }^{1} \mathrm{H}$ (top) and ${ }^{13} \mathrm{C}\left\{{ }^{1} \mathrm{H}\right\}$ APT (bottom) NMR spectra of complex cis-C, $C^{*}-\mathbf{2 e}\left(\mathrm{CD}_{2} \mathrm{Cl}_{2}, 600\right.$ and $151 \mathrm{MHz}$, respectively). 


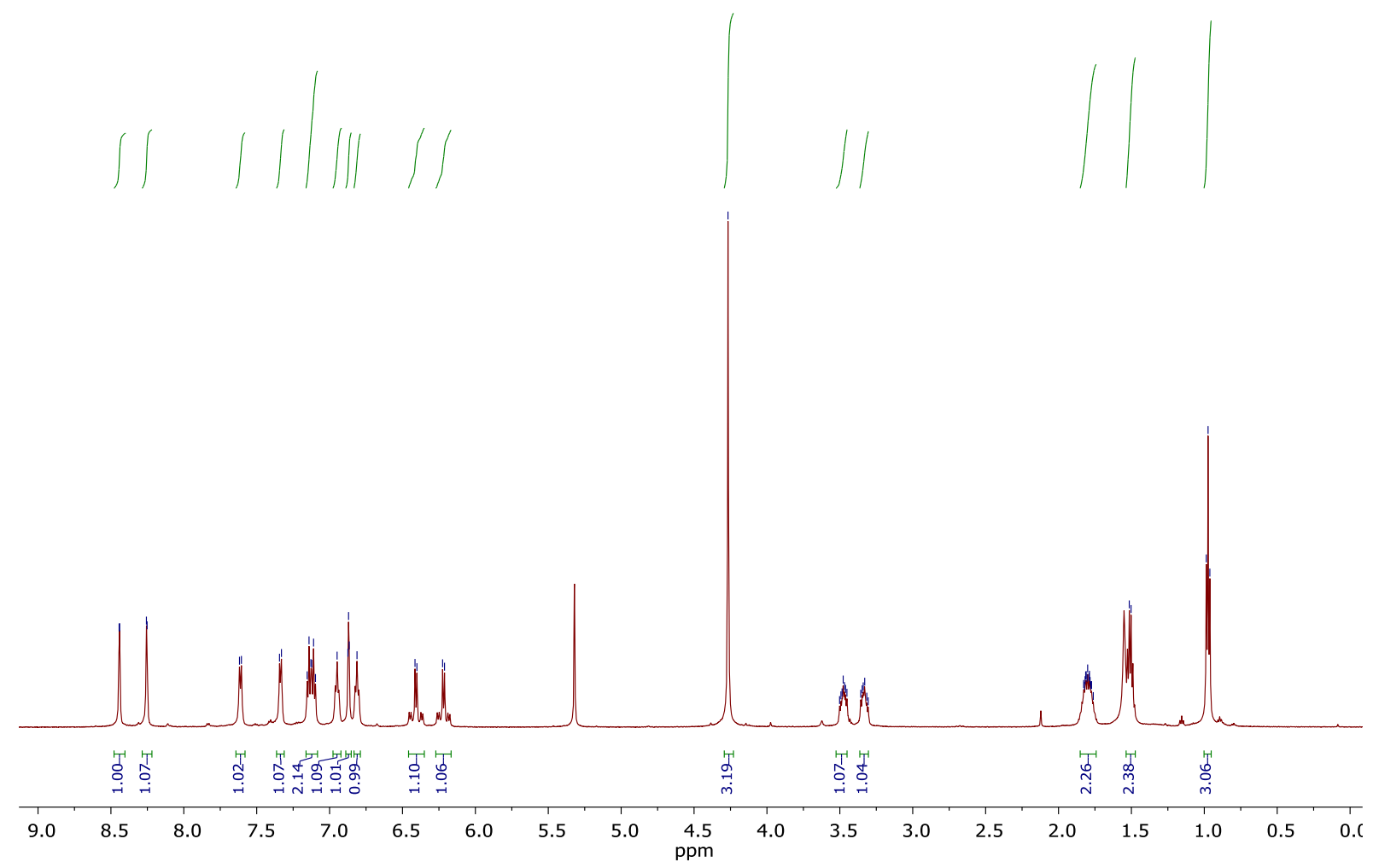

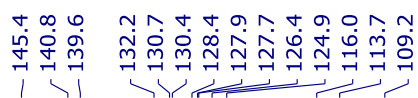

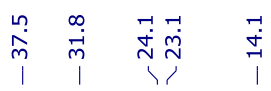

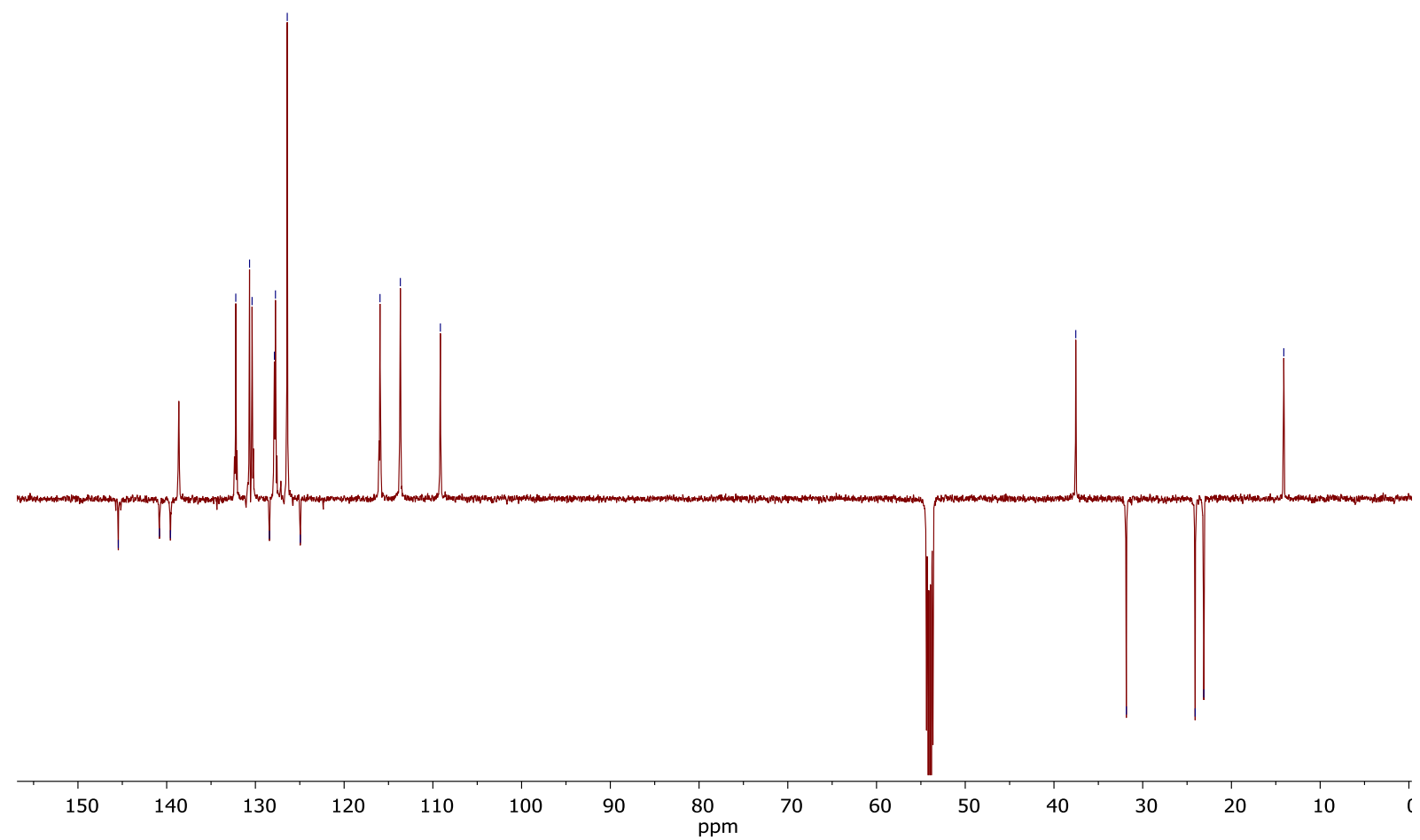

Figure S13. ${ }^{1} \mathrm{H}$ (top) and ${ }^{13} \mathrm{C}\left\{{ }^{1} \mathrm{H}\right\}$ APT (bottom) NMR spectra of complex 3a $\left(\mathrm{CD}_{2} \mathrm{Cl}_{2}, 600\right.$ and 151 $\mathrm{MHz}$, respectively). 


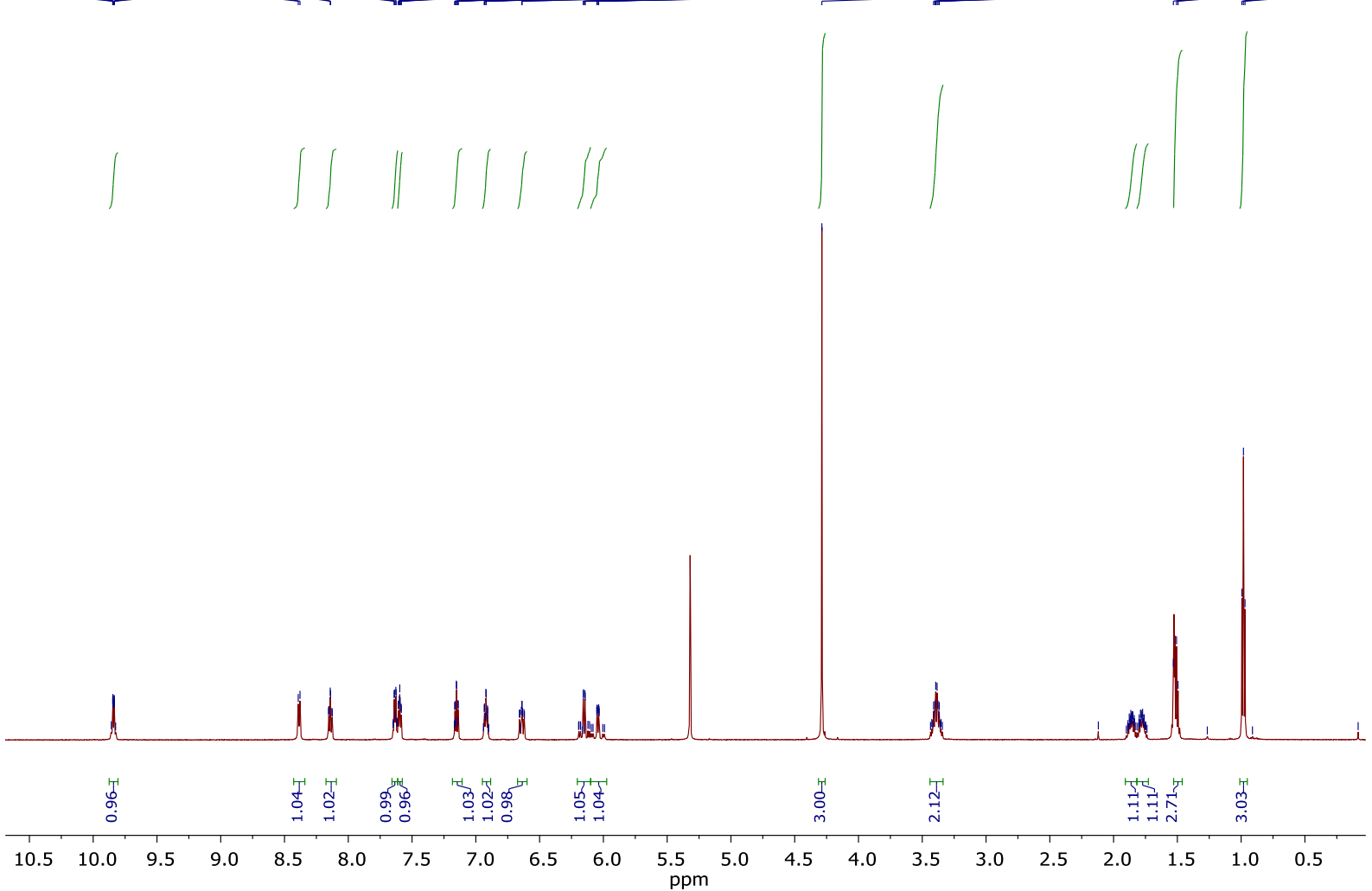

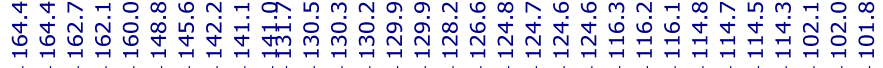

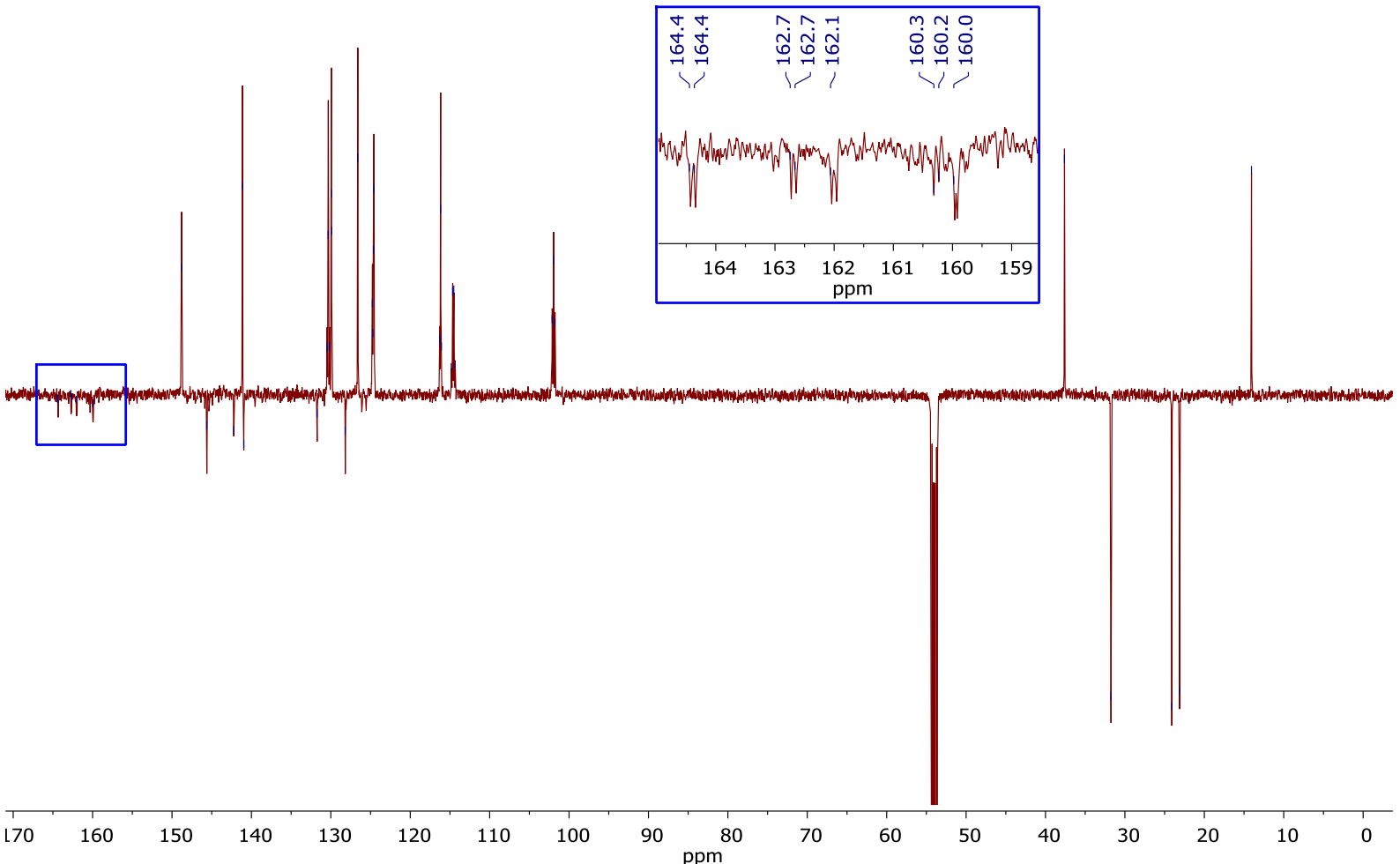

Figure S14. ${ }^{1} \mathrm{H}$ (top) and ${ }^{13} \mathrm{C}\left\{{ }^{1} \mathrm{H}\right\}$ APT (bottom) NMR spectra of complex $\mathbf{3 b}\left(\mathrm{CD}_{2} \mathrm{Cl}_{2}, 600\right.$ and 151 $\mathrm{MHz}$, respectively). 


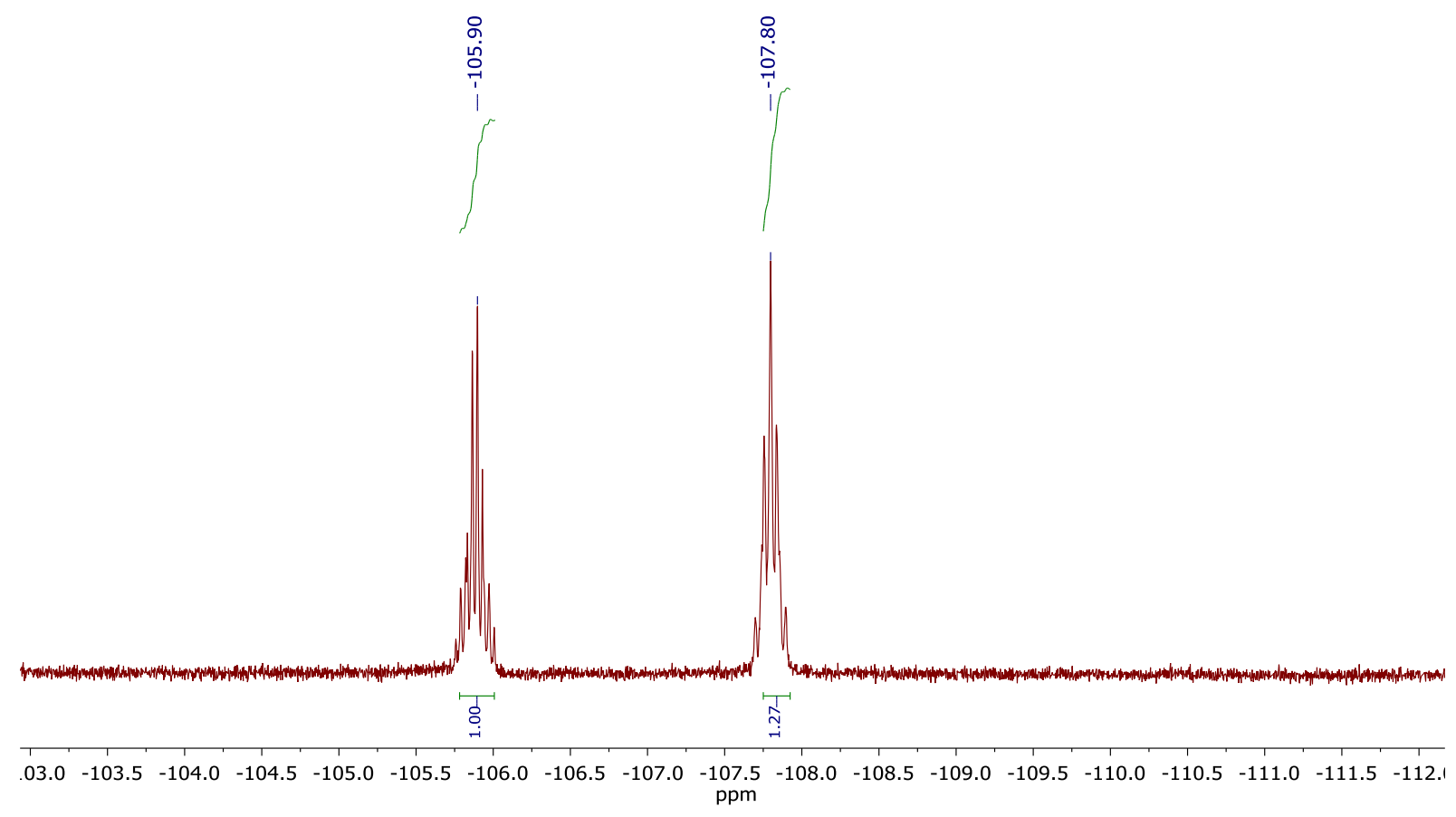

Figure S15. ${ }^{19} \mathrm{~F}$ NMR spectrum of complex $\mathbf{3 b}\left(\mathrm{CD}_{2} \mathrm{Cl}_{2}, 282 \mathrm{MHz}\right)$. 

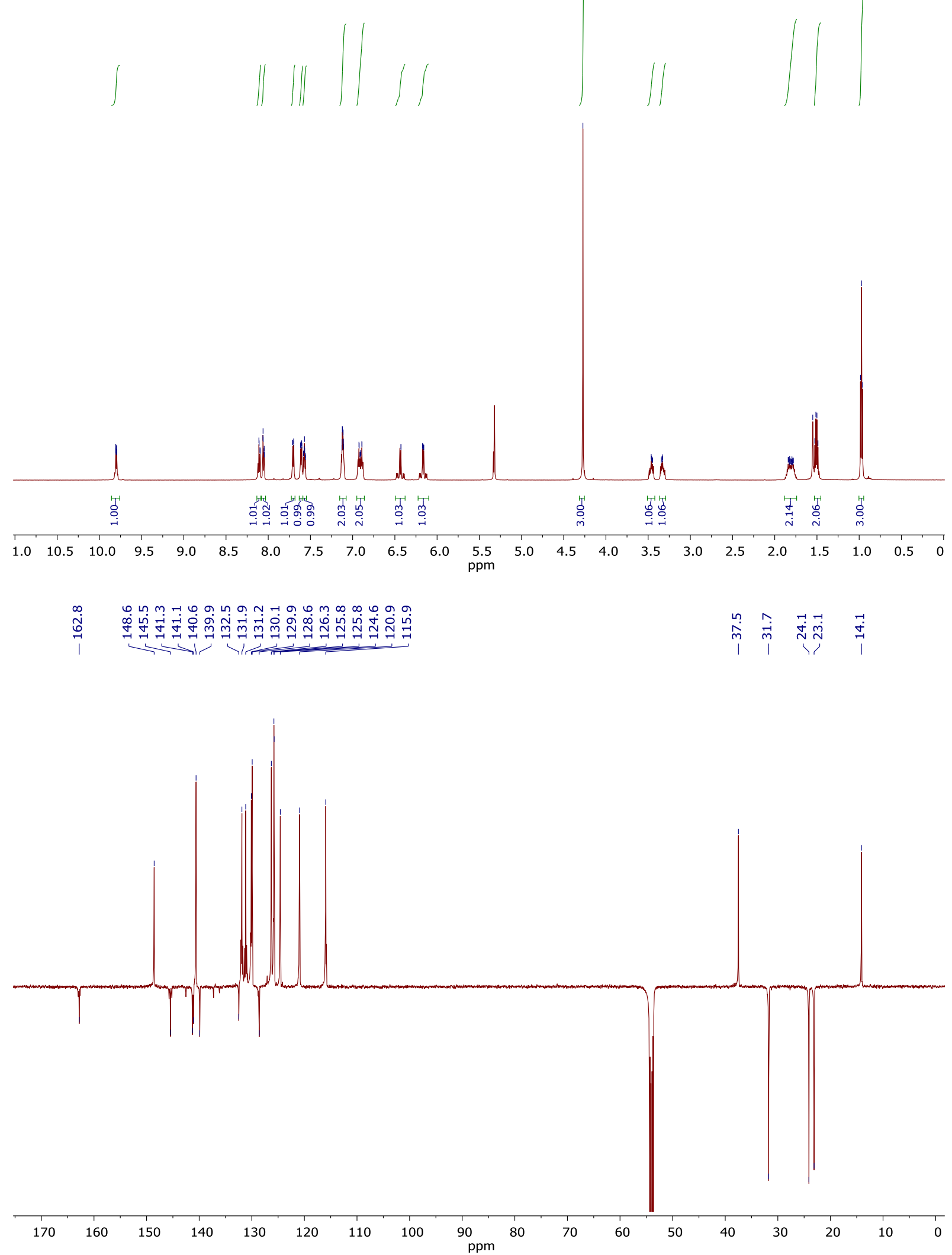

Figure S16. ${ }^{1} \mathrm{H}$ (top) and ${ }^{13} \mathrm{C}\left\{{ }^{1} \mathrm{H}\right\}$ APT (bottom) NMR spectra of complex $3 \mathbf{c}\left(\mathrm{CD}_{2} \mathrm{Cl}_{2}, 600\right.$ and 151 $\mathrm{MHz}$, respectively). 

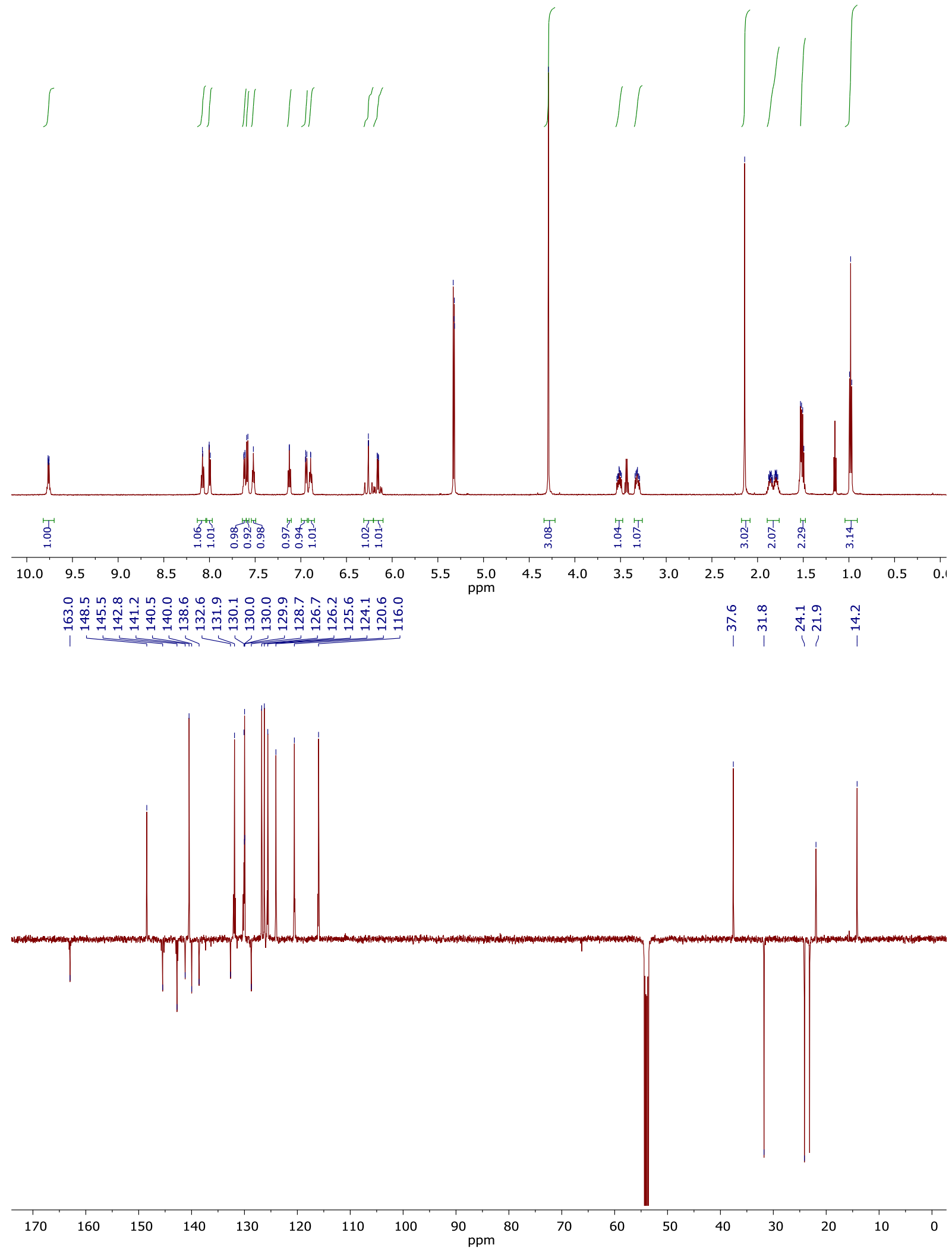

Figure S17. ${ }^{1} \mathrm{H}$ (top) and ${ }^{13} \mathrm{C}\left\{{ }^{1} \mathrm{H}\right\}$ APT (bottom) NMR spectra of complex 3d $\left(\mathrm{CD}_{2} \mathrm{Cl}_{2}, 600\right.$ and 151 $\mathrm{MHz}$, respectively). 

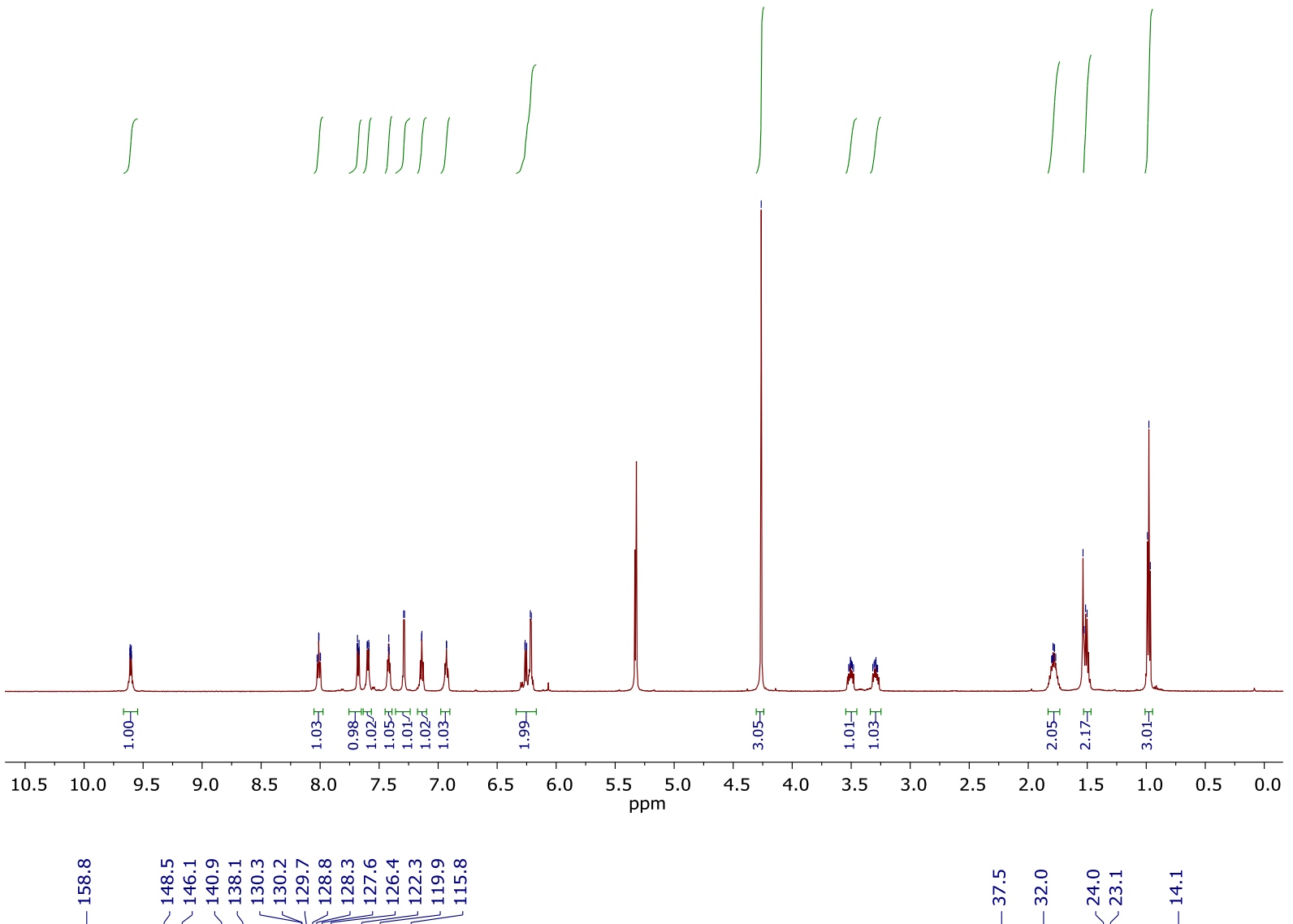

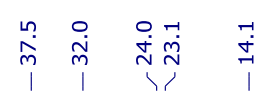

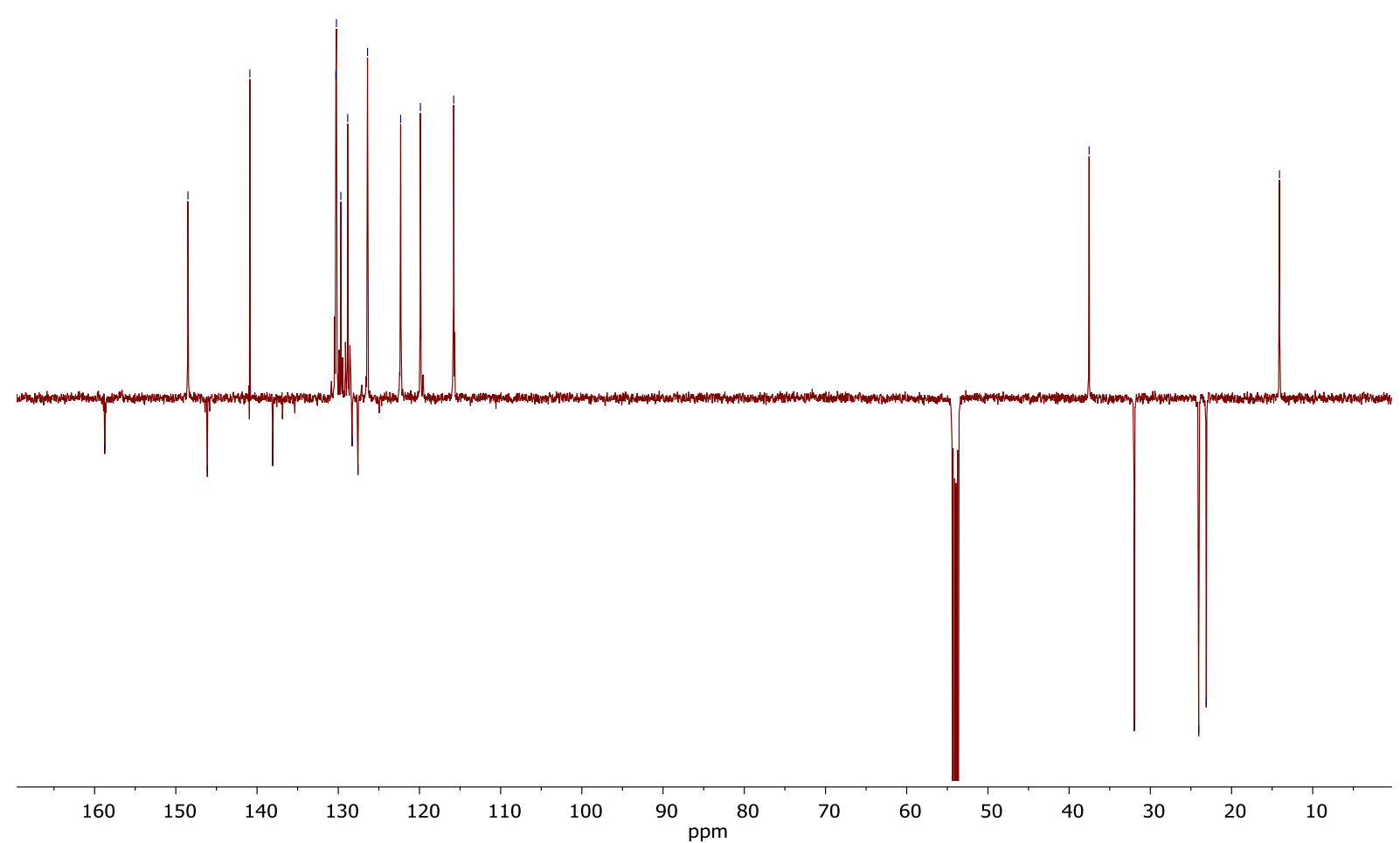

Figure S18. ${ }^{1} \mathrm{H}$ (top) and ${ }^{13} \mathrm{C}\left\{{ }^{1} \mathrm{H}\right\}$ APT (bottom) NMR spectra of complex 3e $\left(\mathrm{CD}_{2} \mathrm{Cl}_{2}, 600\right.$ and 151 $\mathrm{MHz}$, respectively). 


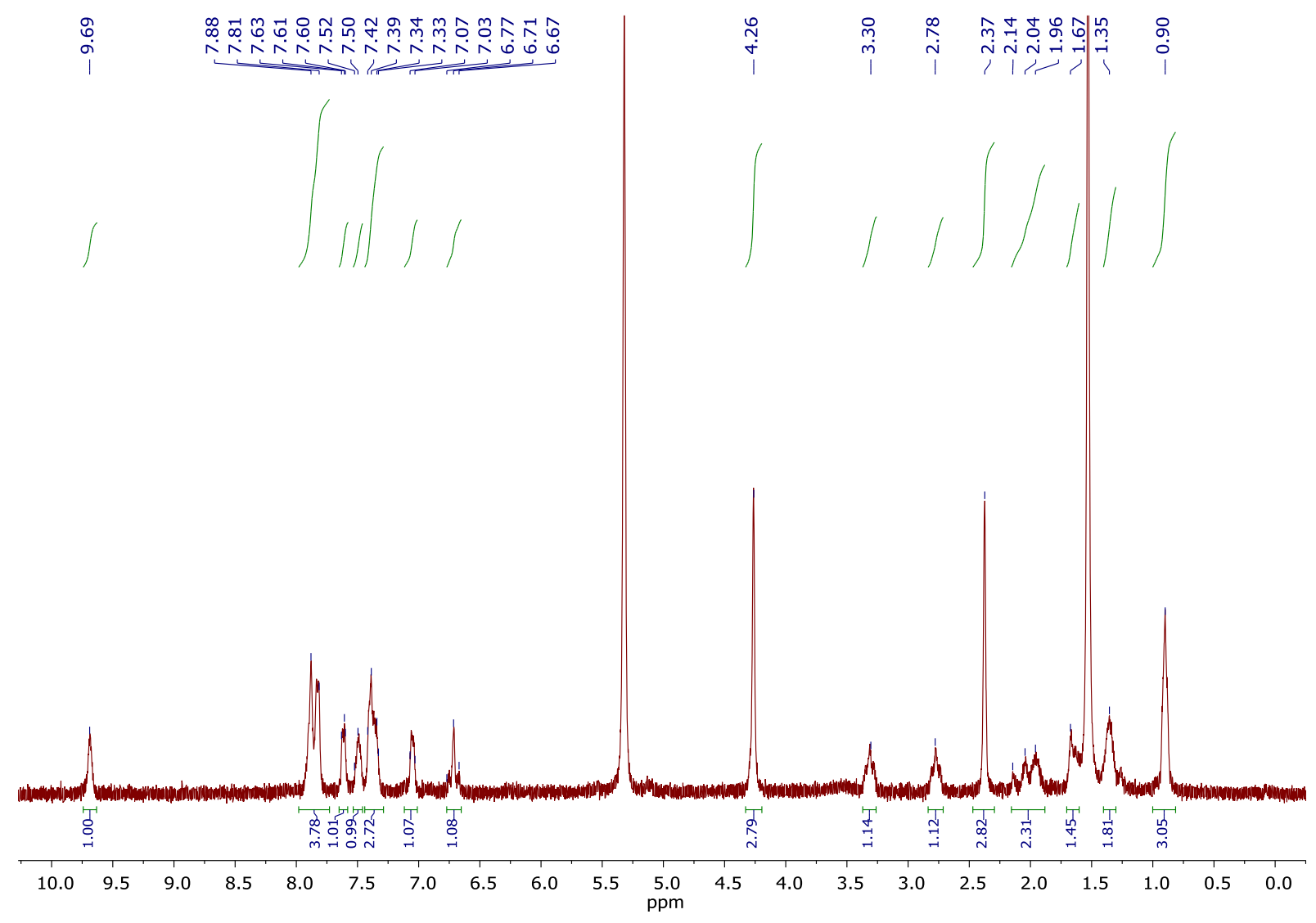

Figure S19. ${ }^{1} \mathrm{H}$ NMR spectrum of complex $4 \mathbf{d}\left(\mathrm{CD}_{2} \mathrm{Cl}_{2}, 400 \mathrm{MHz}\right)$. 

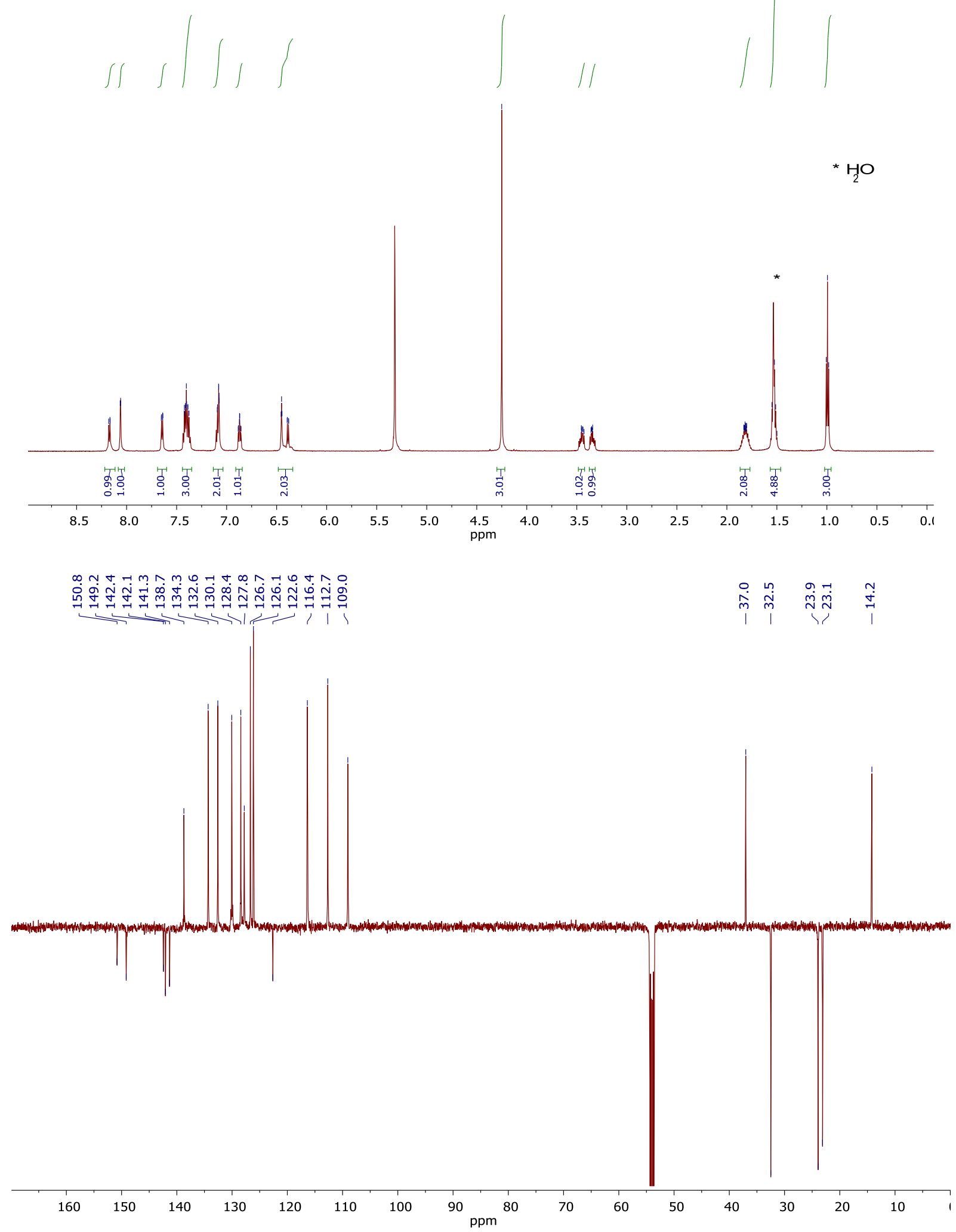

Figure S20. ${ }^{1} \mathrm{H}$ (top) and ${ }^{13} \mathrm{C}\left\{{ }^{1} \mathrm{H}\right\}$ APT (bottom) NMR spectra of complex 5a $\left(\mathrm{CD}_{2} \mathrm{Cl}_{2}, 600\right.$ and 151 $\mathrm{MHz}$, respectively). 


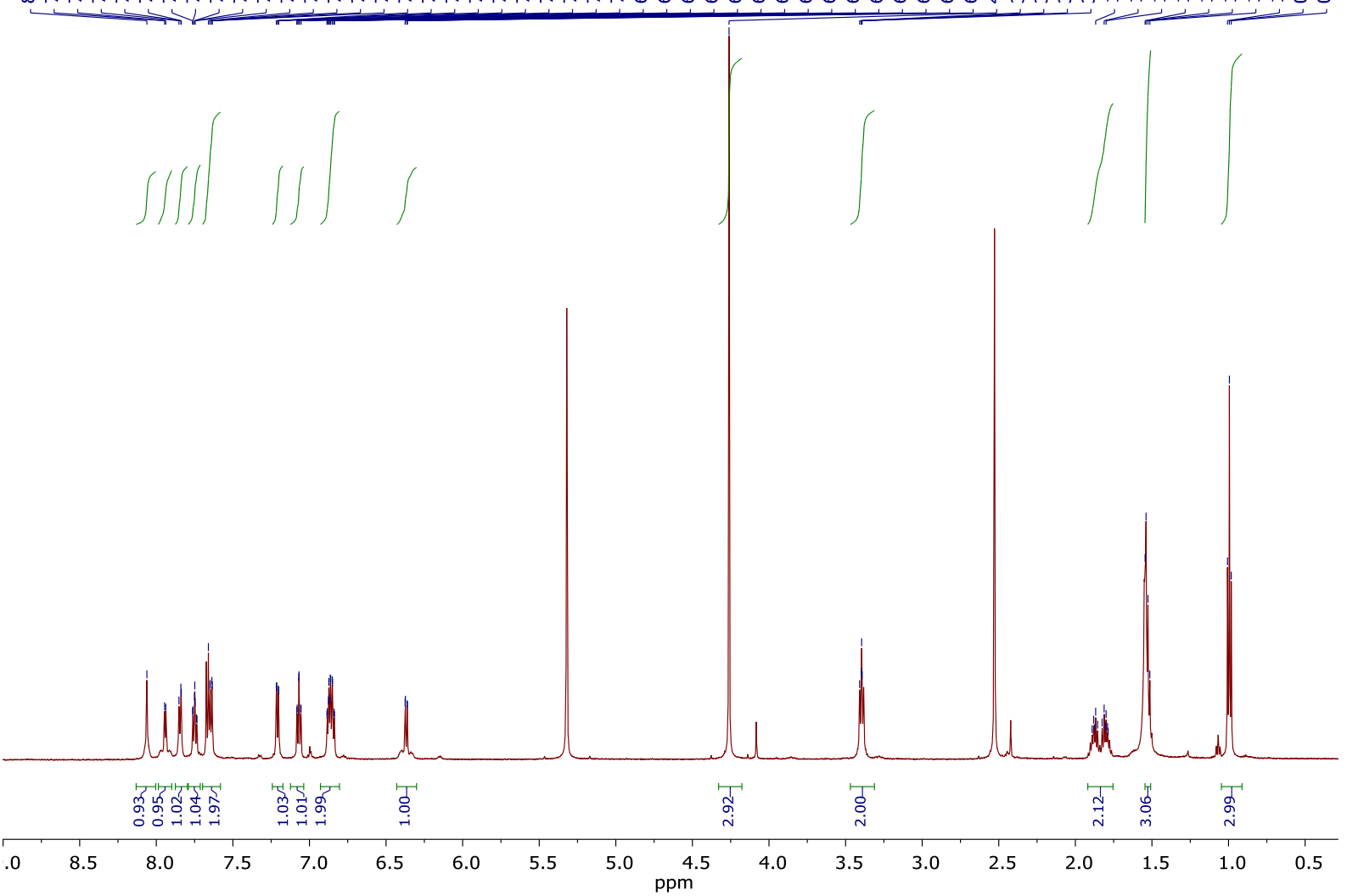

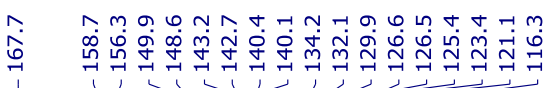

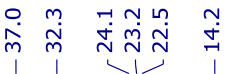

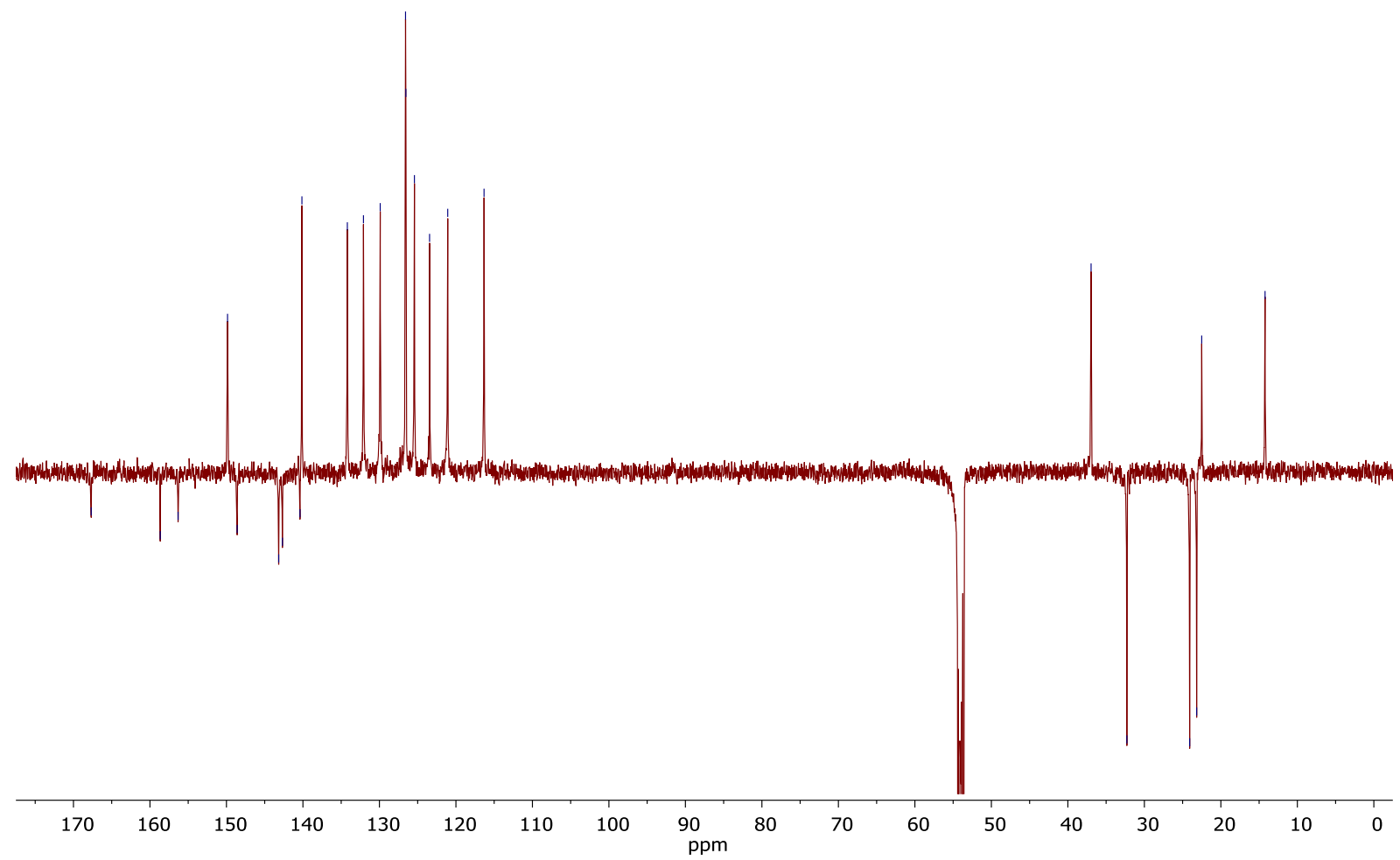

Figure S21. ${ }^{1} \mathrm{H}$ (top) and ${ }^{13} \mathrm{C}\left\{{ }^{1} \mathrm{H}\right\}$ APT (bottom) NMR spectra of complex 5d $\left(\mathrm{CD}_{2} \mathrm{Cl}_{2}, 600\right.$ and 151 $\mathrm{MHz}$, respectively). 


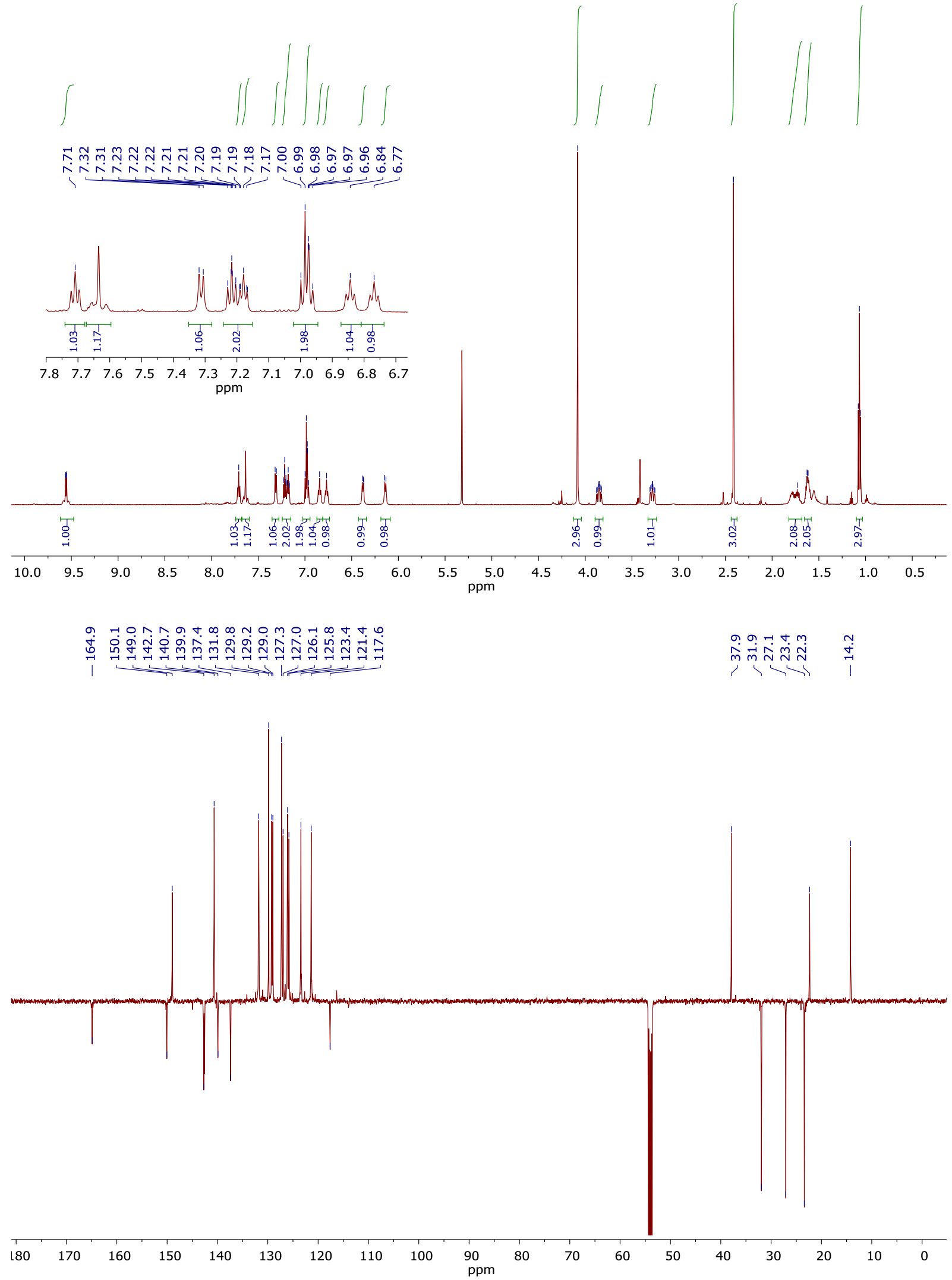

Figure S22. ${ }^{1} \mathrm{H}$ (top) and ${ }^{13} \mathrm{C}\left\{{ }^{1} \mathrm{H}\right\}$ APT (bottom) NMR spectra of complex $6 \mathbf{d}\left(\mathrm{CD}_{2} \mathrm{Cl}_{2}, 600\right.$ and 151 $\mathrm{MHz}$, respectively). 


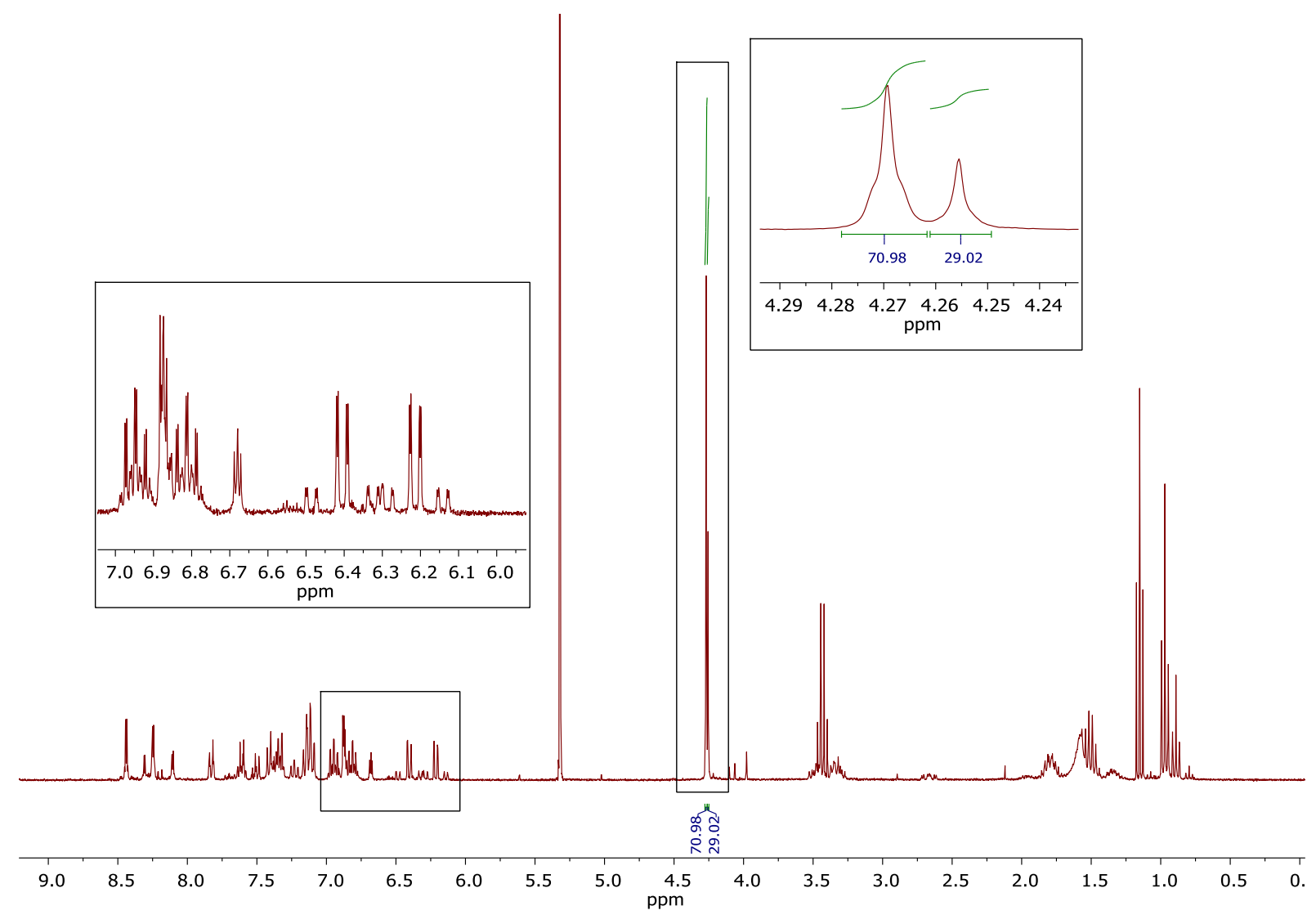

Figure S23. ${ }^{1} \mathrm{H}$ NMR spectrum after oxidation of cis-C, $C^{*}-\mathbf{2 a}$, complexes 3a and 4a in 70:30 ratio $\left(\mathrm{CD}_{2} \mathrm{Cl}_{2}, 300 \mathrm{MHz}\right)$.

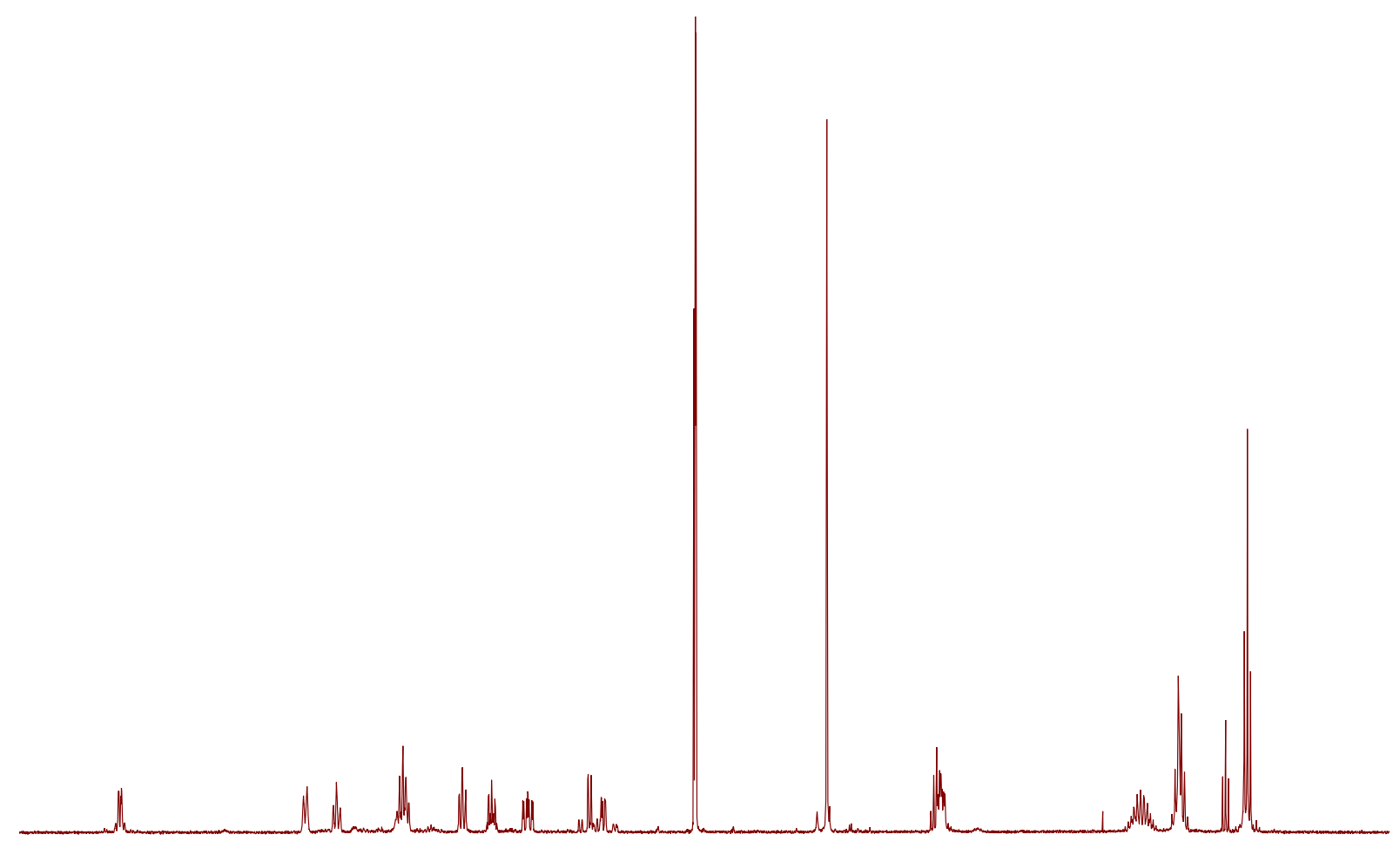

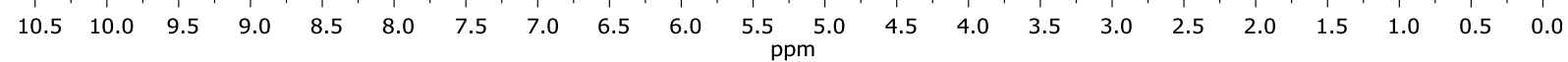

Figure S24. ${ }^{1} \mathrm{H}$ NMR spectrum after oxidation of cis-C, $C^{*}-\mathbf{2 b}\left(\mathrm{CD}_{2} \mathrm{Cl}_{2}, 300 \mathrm{MHz}\right)$. 

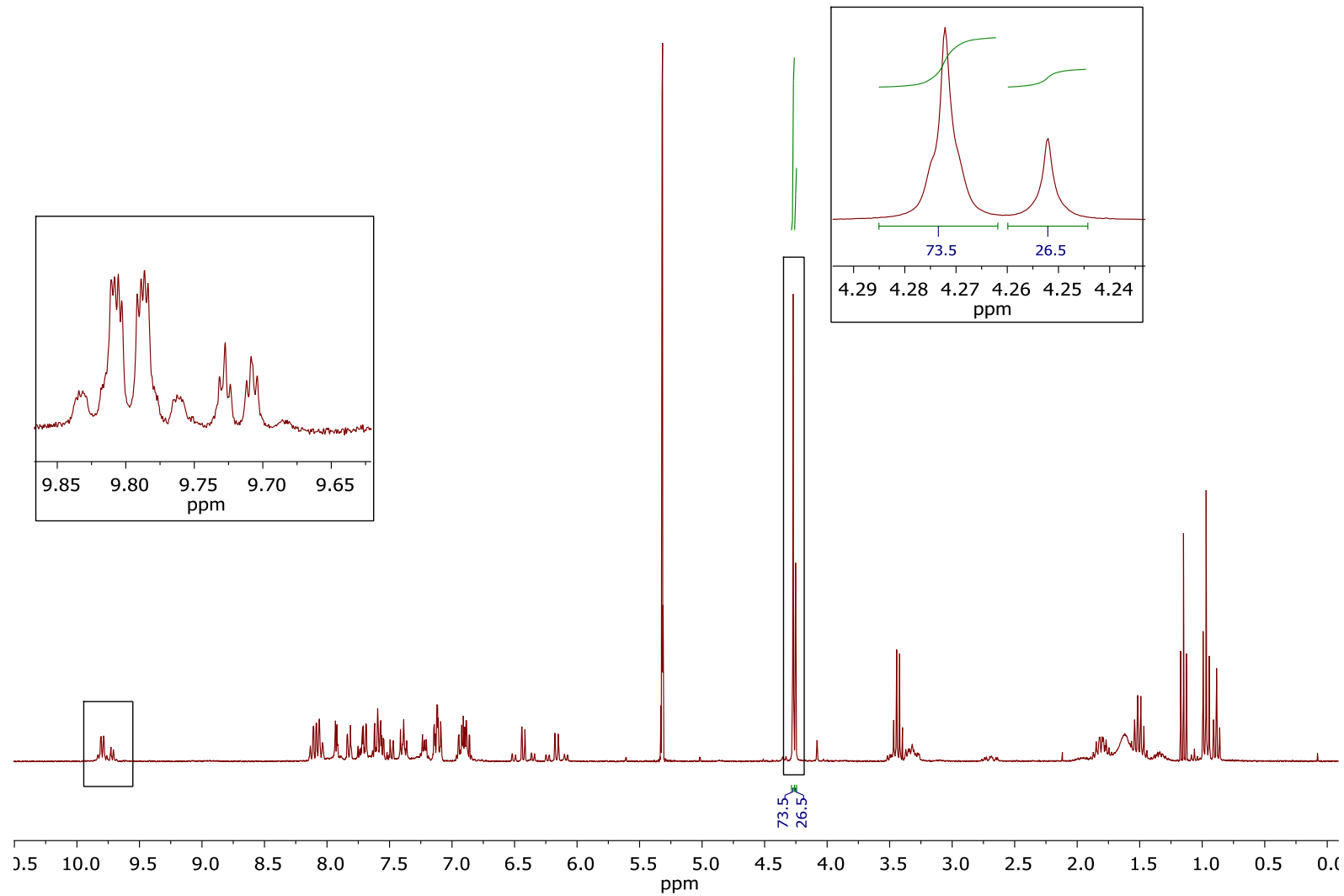

Figure S25. ${ }^{1} \mathrm{H}$ NMR spectrum after oxidation of cis-C, $C^{*}-\mathbf{2 c}$, complexes $\mathbf{3 c}$ and $\mathbf{4 c}$ in $74: 26$ ratio $\left(\mathrm{CD}_{2} \mathrm{Cl}_{2}, 300 \mathrm{MHz}\right)$.

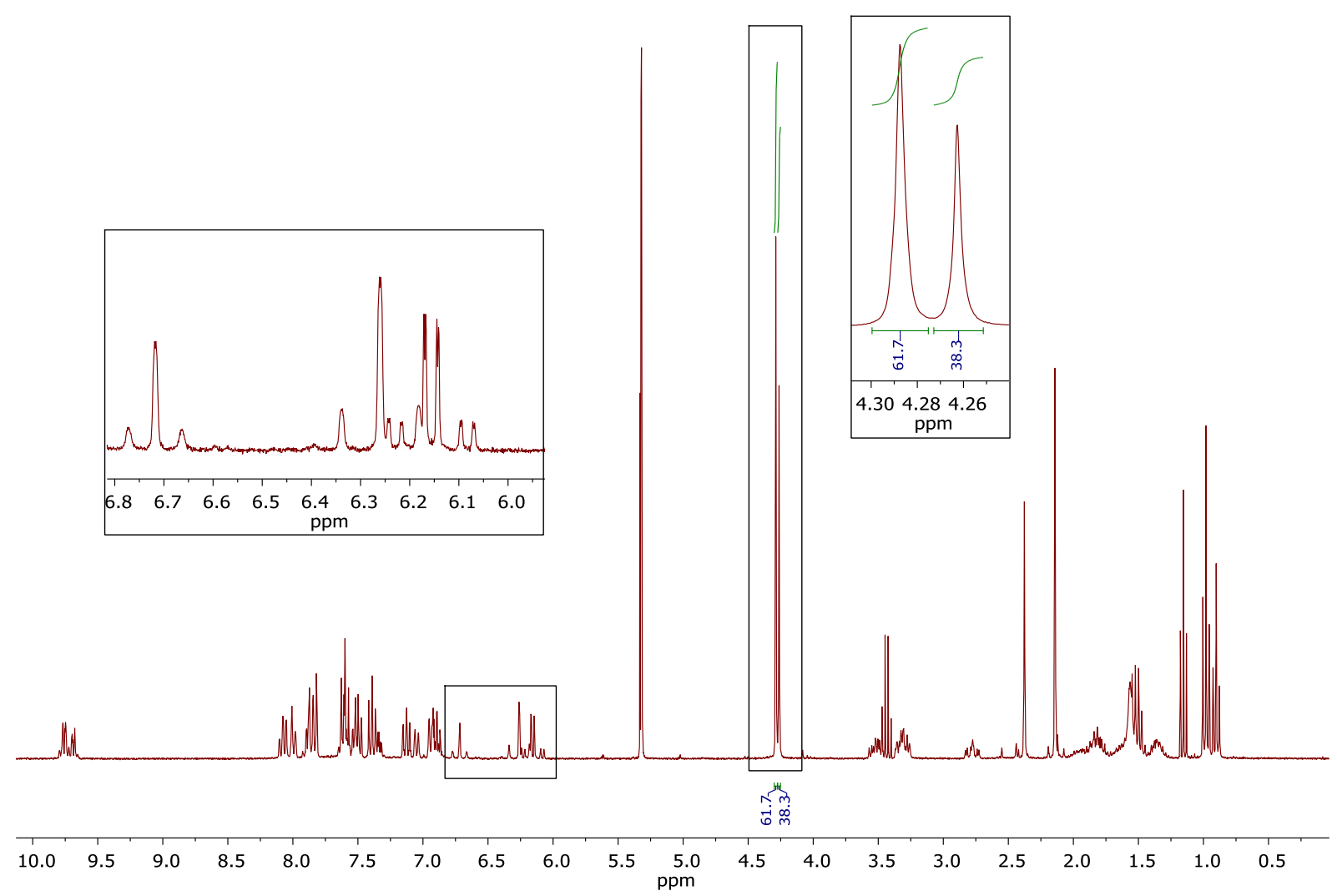

Figure S26. ${ }^{1} \mathrm{H}$ NMR spectrum after oxidation of cis-C, $C^{*}$-2d, complexes $\mathbf{3 d}$ and $\mathbf{4 d}$ in $62: 38$ ratio $\left(\mathrm{CD}_{2} \mathrm{Cl}_{2}, 300 \mathrm{MHz}\right)$. 


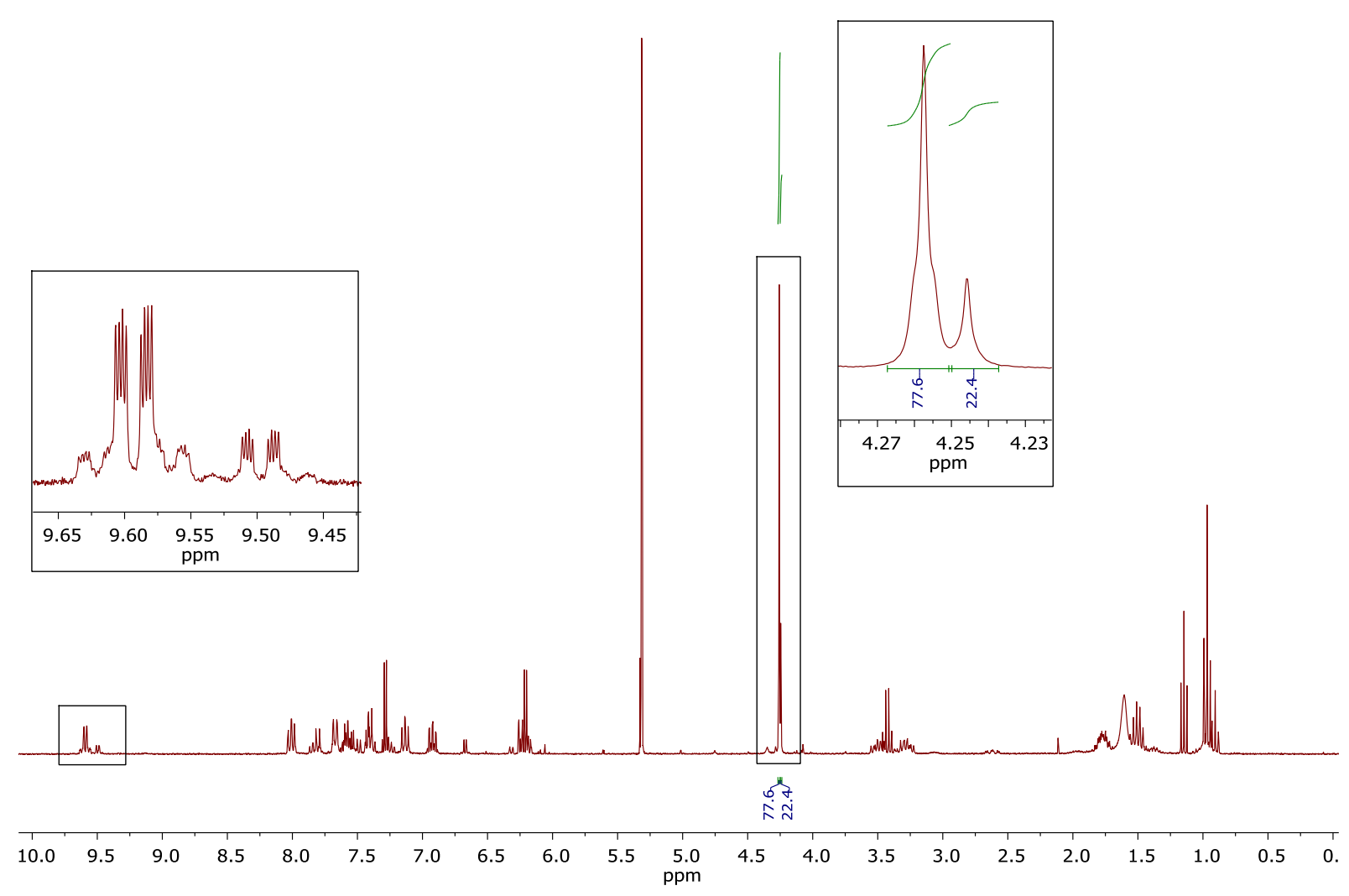

Figure S27. ${ }^{1} \mathrm{H}$ NMR spectrum after oxidation of $c i s-C, C^{*}-\mathbf{2 e}$, complexes $\mathbf{3 e}$ and $\mathbf{4 e}$ in 78:22 ratio $\left(\mathrm{CD}_{2} \mathrm{Cl}_{2}, 300 \mathrm{MHz}\right)$.

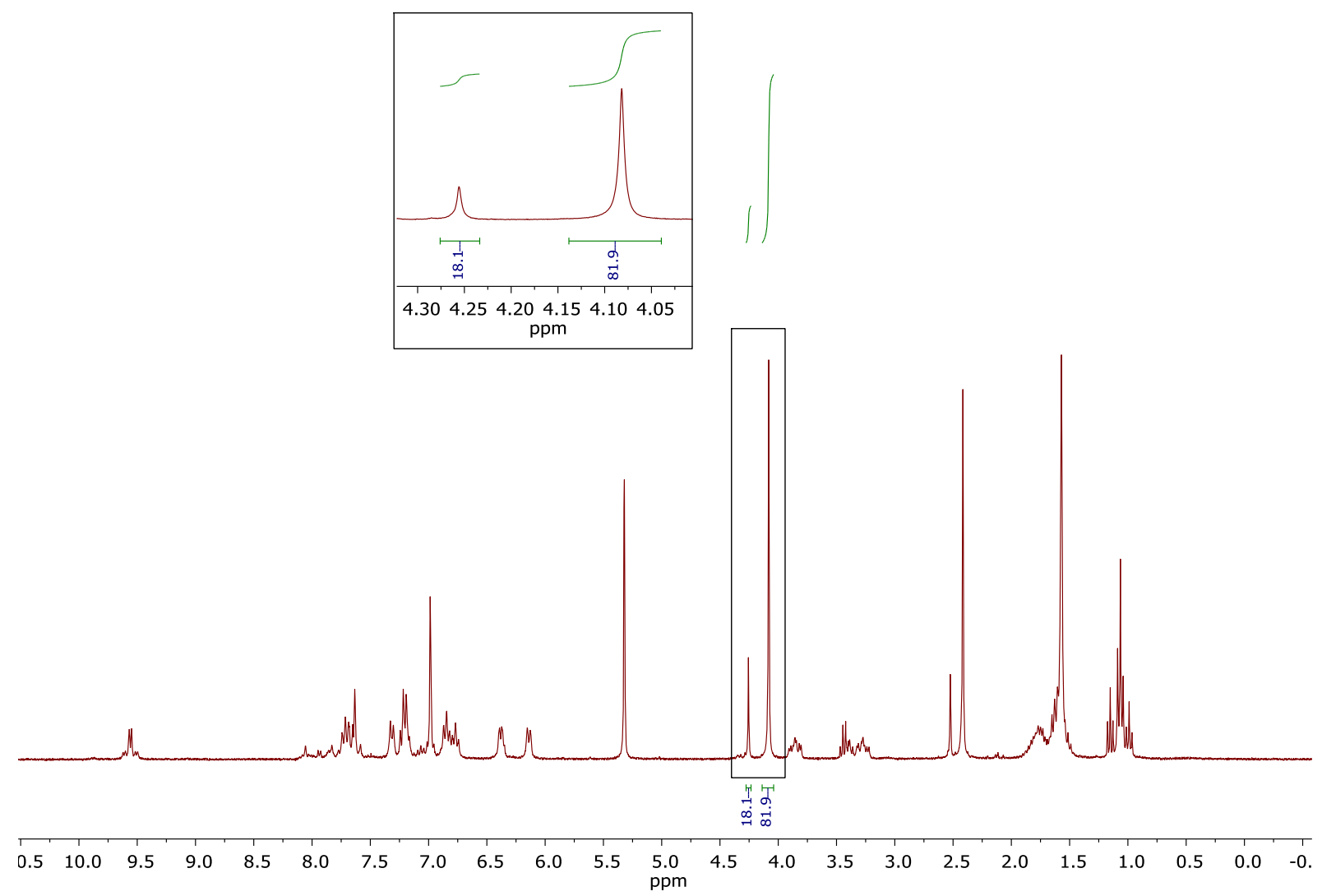

Figure S28. ${ }^{1} \mathrm{H}$ NMR spectrum after oxidation of trans-C, $C^{*}-\mathbf{2 d}$, complexes $\mathbf{5 d}$ and $\mathbf{6 d}$ in 18:82 ratio $\left(\mathrm{CD}_{2} \mathrm{Cl}_{2}, 300 \mathrm{MHz}\right)$. 
Table S2. Molar ratios of complexes 3 and 4 resulting from the oxidative addition of $\mathrm{PhICl}_{2}$ to cis$C, C^{*-2}$ as determined by ${ }^{1} \mathrm{H}$ NMR.

\begin{tabular}{cc}
\hline $\mathrm{C}^{\wedge} \mathrm{N}$ ligand & Molar ratio 3:4 \\
\hline $\mathrm{ppz}$ & $70: 30$ \\
dfppy & $95: 5$ \\
ppy & $74: 26$ \\
tpy & $62: 38$ \\
thpy & $78: 22$ \\
\hline
\end{tabular}




\section{Excitation and emission spectra}
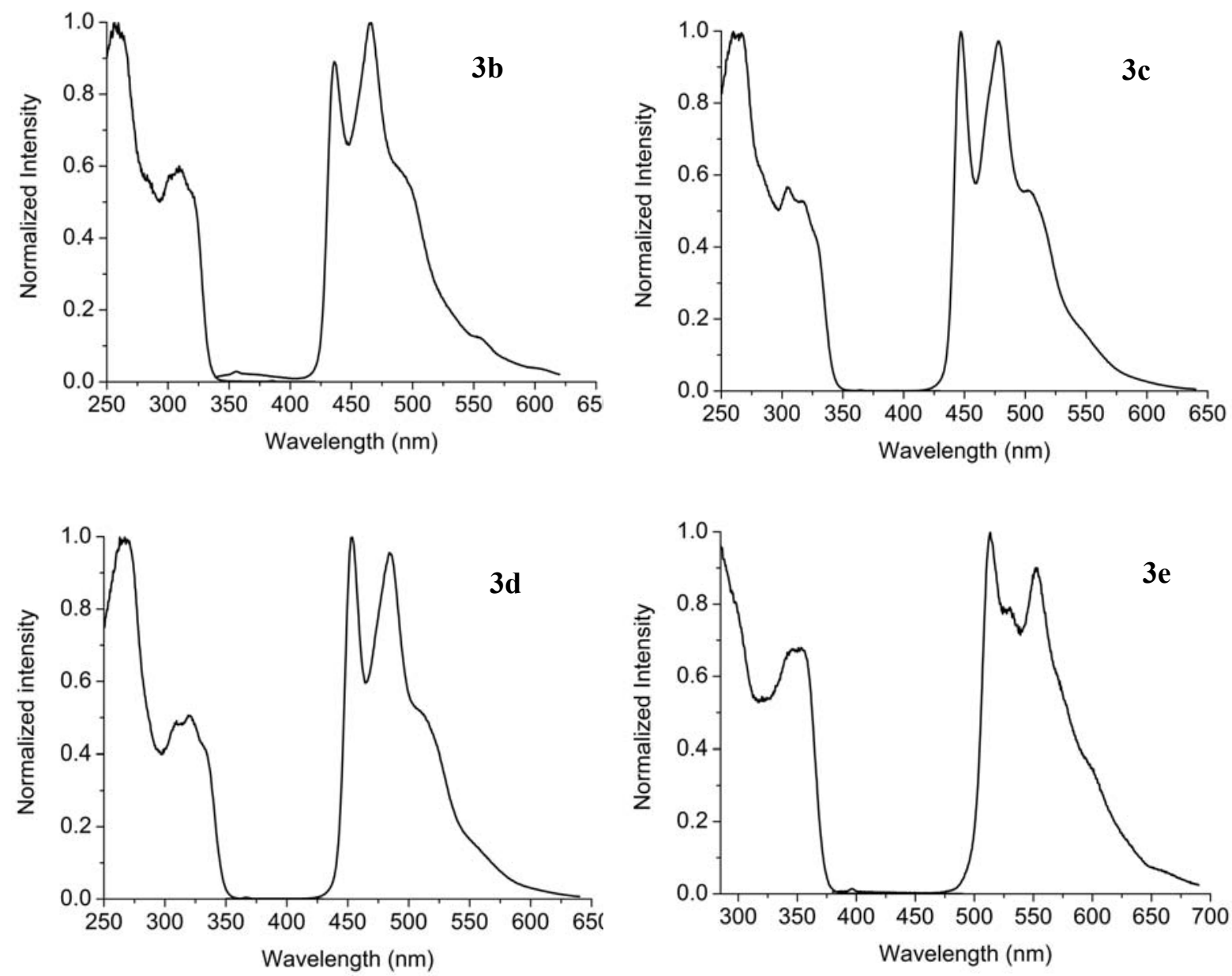

Figure S29. Excitation and emission spectra of complexes 3b-e in $\mathrm{CH}_{2} \mathrm{Cl}_{2}$ at $298 \mathrm{~K}$. 

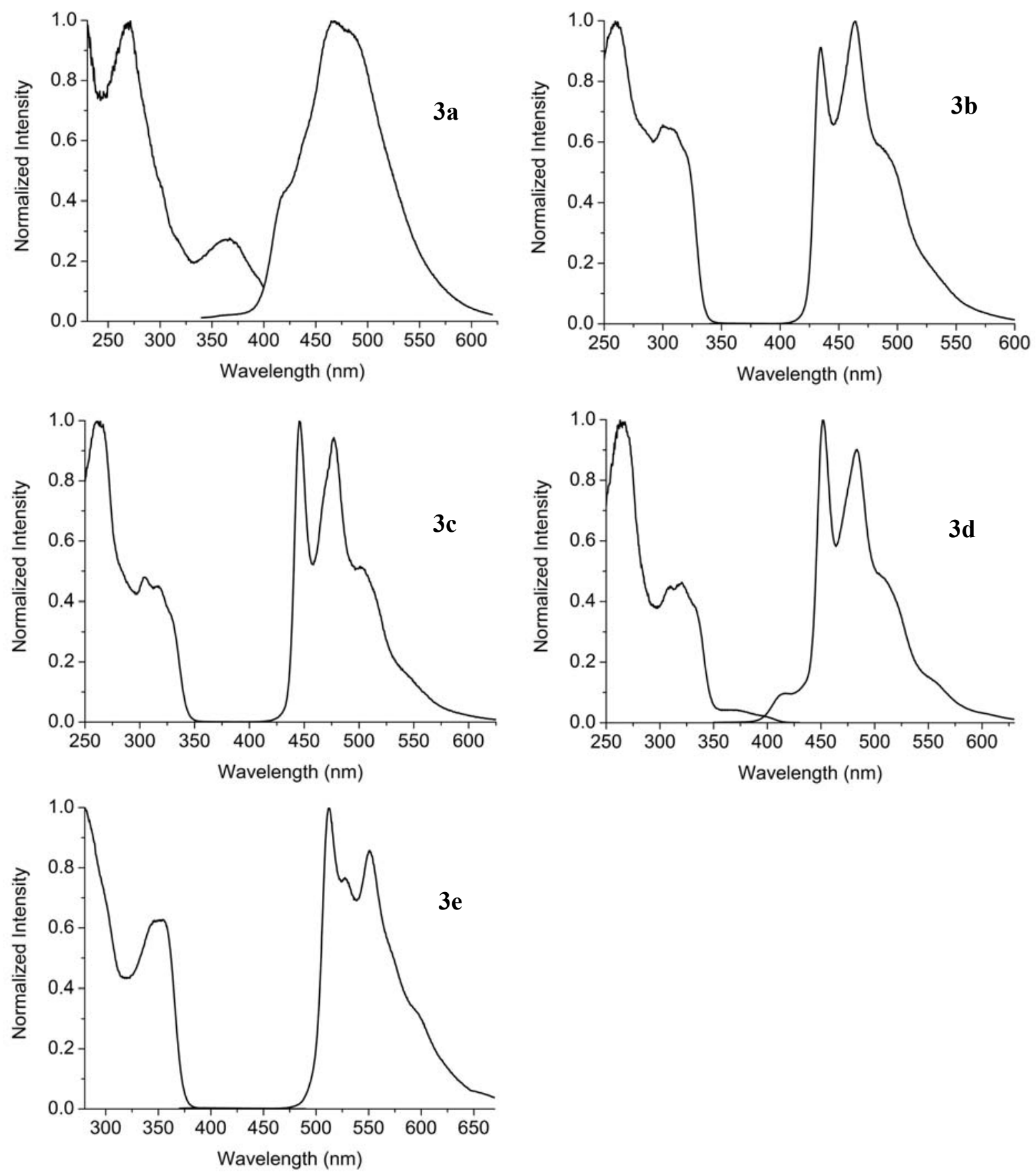

Figure S30. Excitation and emission spectra of complexes 3a-e in PMMA matrices (2 wt \%) at $298 \mathrm{~K}$. 

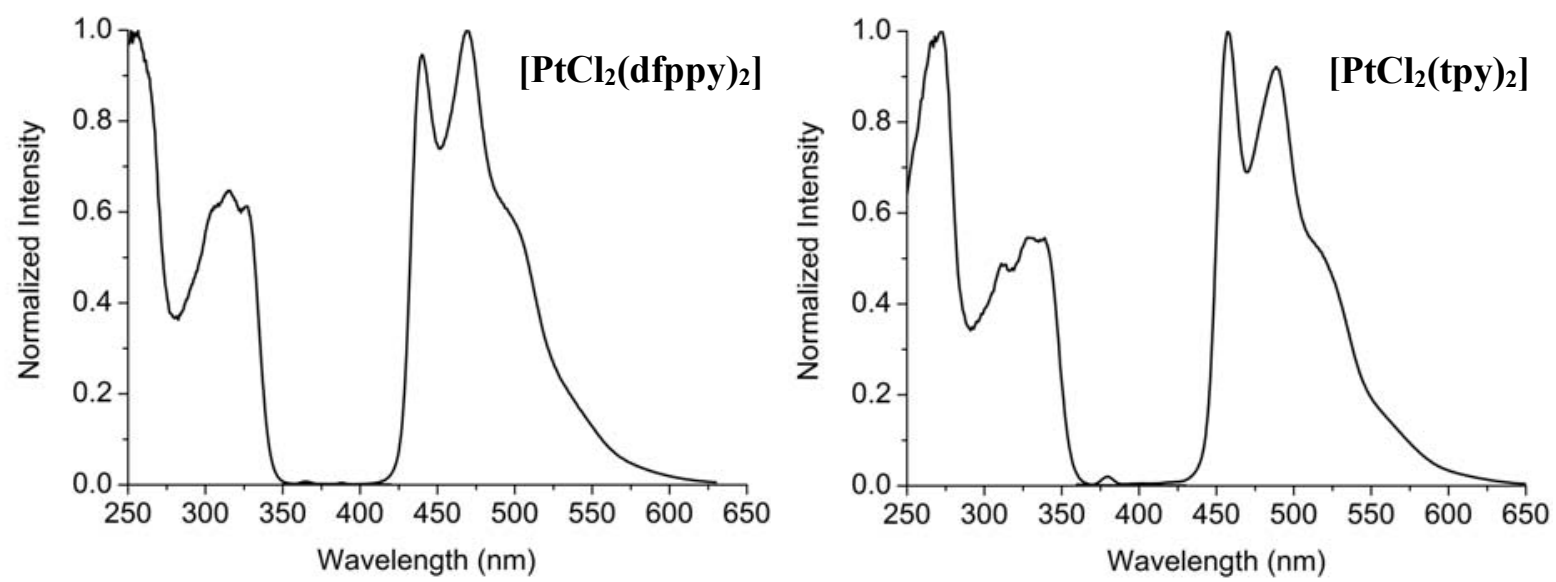

Figure S31. Excitation and emission spectra of complexes (OC-6-33)-[ $\left.\mathrm{PtCl}_{2}(\mathrm{dfppy})_{2}\right]$ and (OC-6-33)$\left[\mathrm{PtCl}_{2}(\text { tpy })_{2}\right]$ in $\mathrm{CH}_{2} \mathrm{Cl}_{2}$ at $298 \mathrm{~K}$.
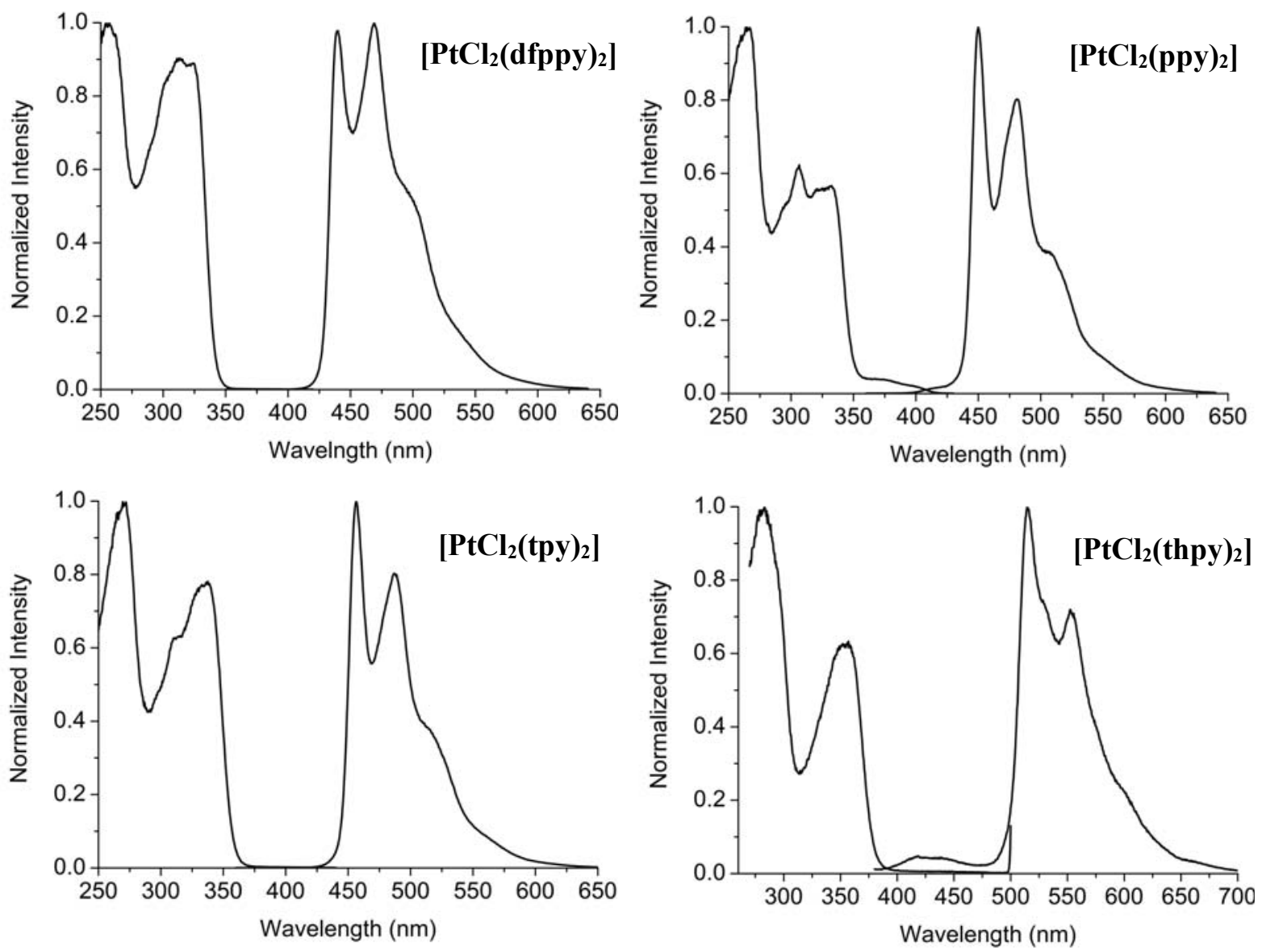

Figure S32. Excitation and emission spectra of complexes $(O C-6-33)-\left[\mathrm{PtCl}_{2}\left(\mathrm{C}^{\wedge} \mathrm{N}\right)_{2}\right]\left(\mathrm{C}^{\wedge} \mathrm{N}=\mathrm{dfppy}\right.$, ppy, tpy, thpy) in PMMA matrices (2 wt\%) at $298 \mathrm{~K}$. 


\section{Computational methods}

DFT calculations were performed with Gaussian $09,{ }^{5}$ using the B3LYP functional ${ }^{6,7}$ together with the $6-31 \mathrm{G}^{* * 8,9}$ basis set for the light atoms and the LANL2DZ ${ }^{10}$ basis set and effective core potential for the Pt atom. Geometry optimizations were carried out without symmetry restrictions, using "tight" convergence criteria and "ultrafine" integration grid. Vertical excitation energies were obtained from TDDFT calculations at the ground-state geometries. Triplet-state geometry optimizations were performed following a published strategy. ${ }^{11}$ The solvent effect $\left(\mathrm{CH}_{2} \mathrm{Cl}_{2}\right)$ was accounted for in all cases by using the integral equation formalism variant of the polarizable continuum solvation model (IEFPCM). ${ }^{12}$ The optimized geometries were confirmed as minima on the potential energy surface by performing frequency calculations (zero imaginary frequencies). Natural spin densities were obtained from natural population analyses using the NBO 5.9 program. ${ }^{13}$ 


\section{Computational data}

\subsection{Complex 3d}

Table S3. Fragment contributions (\%; from atomic orbital contributions) to the frontier orbitals of $\mathbf{3 d}$ in $\mathrm{CH}_{2} \mathrm{Cl}_{2}$ solution.

\begin{tabular}{c|c|ccccc}
\hline energy (a.u.) & number & L1 & L2 & L3 & L4 & Pt \\
\hline-0.010 & 134 (LUMO+5) & 12 & 83 & 0 & 0 & 5 \\
-0.037 & 133 (LUMO+4) & 19 & 36 & 2 & 5 & 39 \\
-0.040 & 132 (LUMO+3) & 64 & 14 & 4 & 2 & 16 \\
-0.044 & 131 (LUMO+2) & 62 & 15 & 4 & 4 & 15 \\
-0.066 & $130($ LUMO+1) & 83 & 12 & 0 & 2 & 4 \\
-0.069 & $129($ LUMO) & 11 & 84 & 1 & 0 & 4 \\
-0.231 & $128($ HOMO) & 92 & 0 & 3 & 0 & 4 \\
-0.243 & 127 (HOMO-1) & 0 & 51 & 5 & 35 & 8 \\
-0.247 & $126($ HOMO-2) & 59 & 4 & 26 & 2 & 9 \\
-0.251 & 125 (HOMO-3) & 5 & 14 & 14 & 59 & 7 \\
-0.252 & $124($ HOMO-4) & 14 & 25 & 13 & 45 & 4 \\
-0.254 & $123($ HOMO-5) & 22 & 7 & 38 & 30 & 3 \\
\hline
\end{tabular}

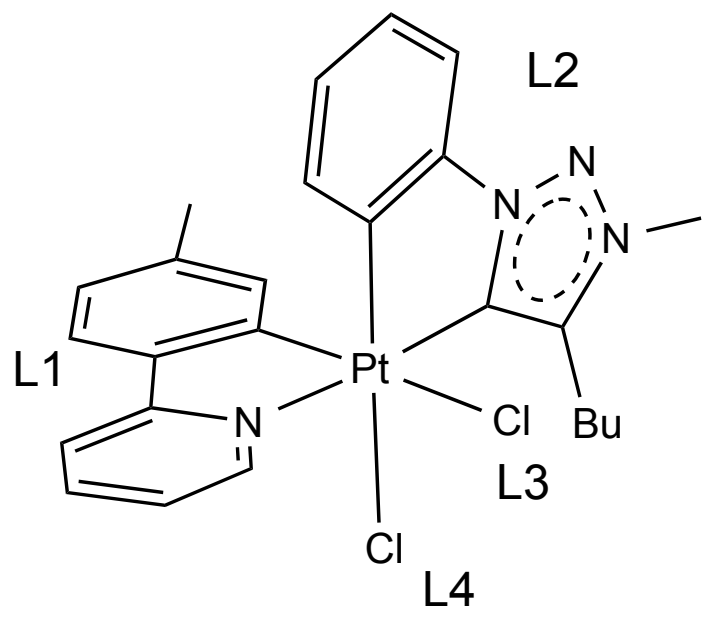

Figure S33. Ligand numbering in complex 3d. 


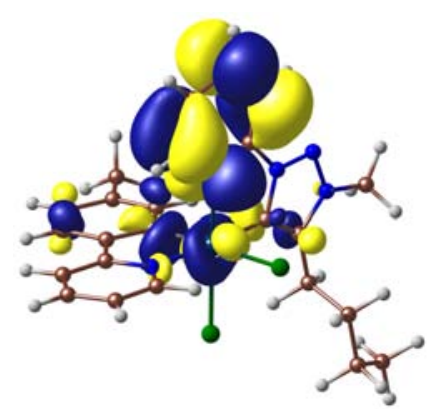

LUMO+5

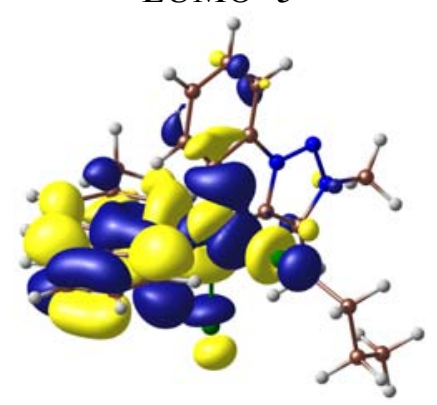

LUMO+2

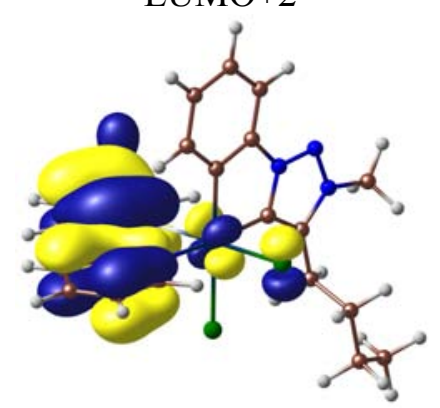

HOMO

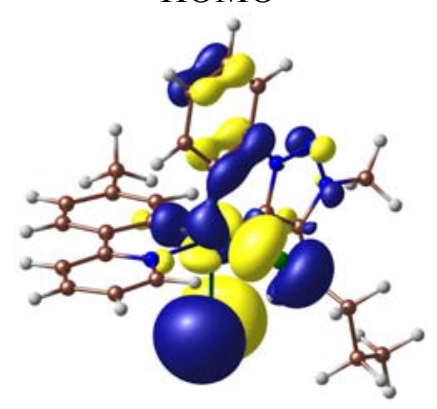

HOMO-3

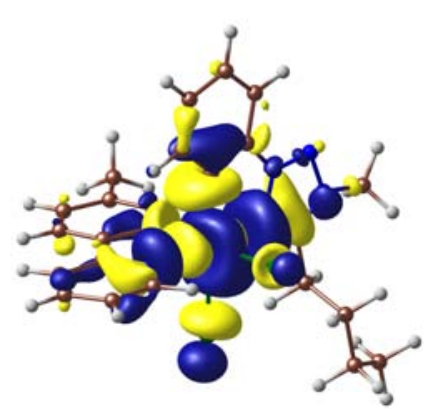

LUMO+4

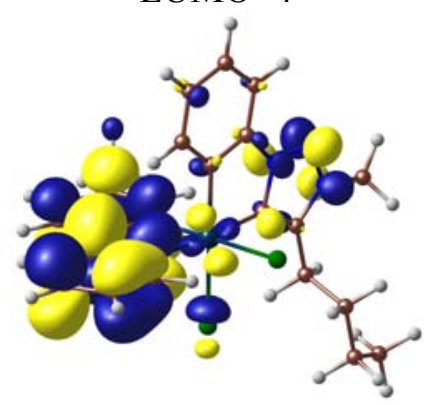

LUMO+1

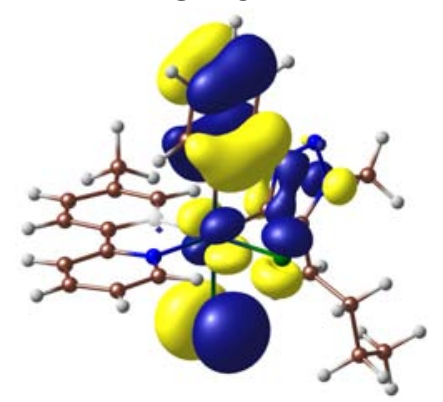

HOMO-1

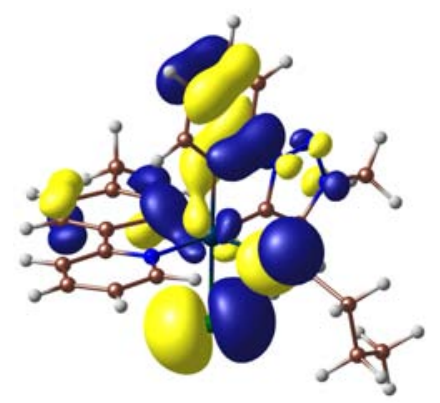

HOMO-4

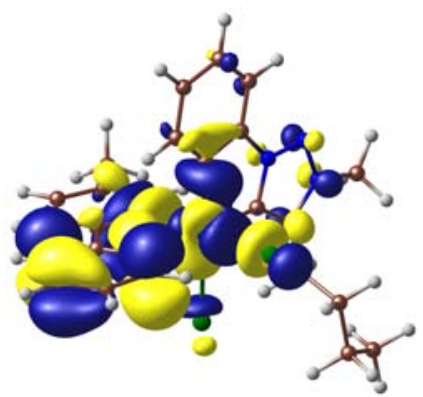

LUMO+3

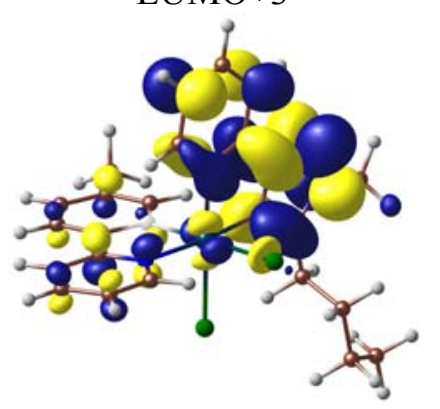

LUMO

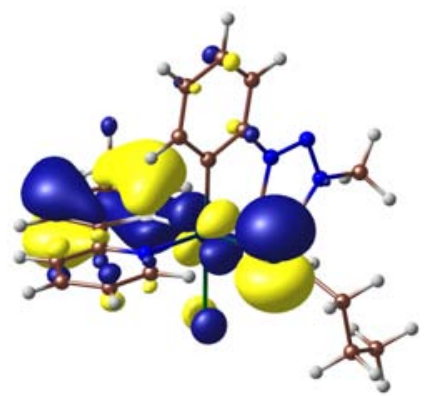

HOMO-2

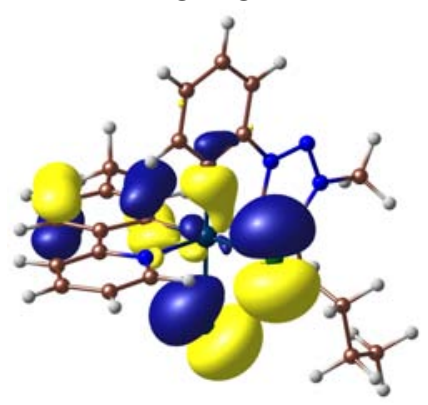

HOMO-5

Figure S34. Molecular orbital isosurfaces of 3d $\left(0.03 \mathrm{e} \mathrm{bohr}^{-3}\right)$. 


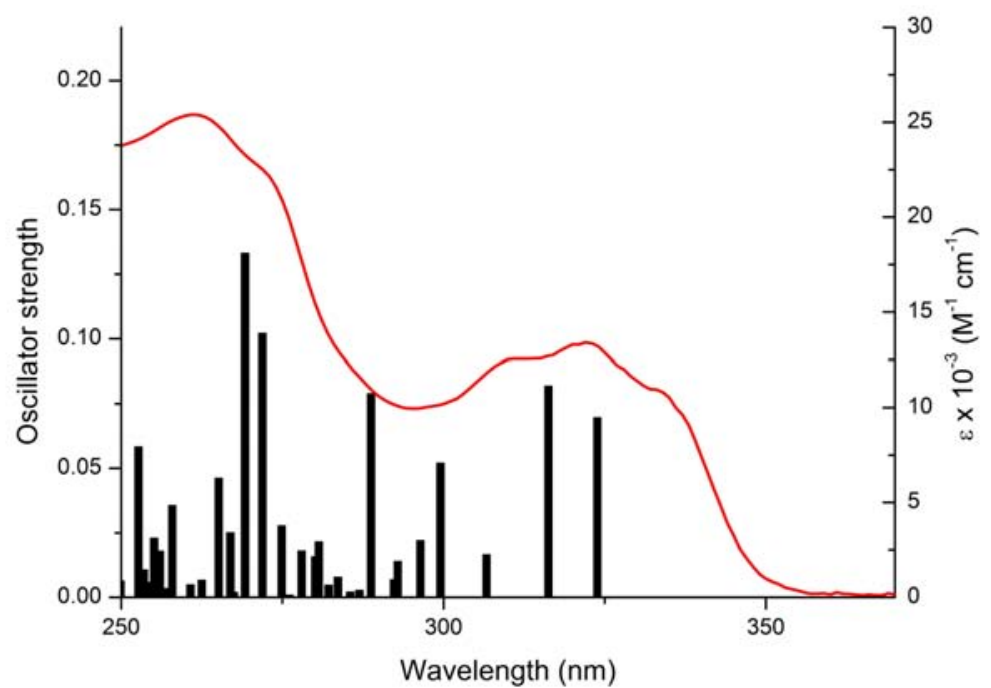

Figure S35. Calculated stick absorption spectrum of $\mathbf{3 d}$ compared with the experimental spectrum in $\mathrm{CH}_{2} \mathrm{Cl}_{2}$ solution $\left(c a .1 \times 10^{-5} \mathrm{M}\right)$ at $298 \mathrm{~K}$.

Table S4. Selected vertical singlet excitations of 3d from TDDFT calculations at the ground state geometry in $\mathrm{CH}_{2} \mathrm{Cl}_{2}$ solution.

\begin{tabular}{|c|c|c|c|c|}
\hline State & monoexcitations & AE/eV & $\lambda / \mathbf{n m}$ & Oscillator strength \\
\hline S1 & $\begin{array}{l}126->129(4 \%) \\
128->129(72 \%) \\
128->130(10 \%) \\
128->131(5 \%)\end{array}$ & 3.829 & 323.9 & 0.0697 \\
\hline S2 & $\begin{array}{l}128->129(16 \%) \\
128->130(78 \%)\end{array}$ & 3.920 & 316.3 & 0.0819 \\
\hline S3 & $\begin{array}{l}127->129(67 \%) \\
127->130(10 \%) \\
127->131(4 \%) \\
128->129(4 \%) \\
128->130(3 \%)\end{array}$ & 4.043 & 306.7 & 0.0166 \\
\hline S4 & $\begin{array}{l}127->129(18 \%) \\
127->130(69 \%)\end{array}$ & 4.140 & 299.5 & 0.0521 \\
\hline S5 & $\begin{array}{l}123->129(6 \%) \\
125->130(2 \%) \\
126->129(57 \%) \\
126->131(5 \%) \\
126->132(3 \%) \\
127->129(4 \%) \\
127->130(3 \%) \\
128->129(6 \%) \\
128->131(4 \%) \\
128->132(5 \%)\end{array}$ & 4.183 & 296.4 & 0.0221 \\
\hline S6 & $\begin{array}{l}124->129(7 \%) \\
124->130(8 \%) \\
125->129(18 \%) \\
125->130(18 \%) \\
126->130(23 \%) \\
128->131(12 \%) \\
128->132(2 \%)\end{array}$ & 4.233 & 292.9 & 0.014 \\
\hline S7 & $\begin{array}{l}123->130(4 \%) \\
124->129(3 \%)\end{array}$ & 4.242 & 292.3 & 0.0069 \\
\hline
\end{tabular}




\begin{tabular}{|c|c|c|c|c|}
\hline & $\begin{array}{l}125->129(11 \%) \\
125->130(12 \%) \\
126->129(16 \%) \\
126->130(4 \%) \\
128->131(30 \%) \\
128->132(8 \%)\end{array}$ & & & \\
\hline S8 & $\begin{array}{l}123->129(8 \%) \\
125->130(5 \%) \\
126->129(5 \%) \\
126->130(51 \%) \\
128->130(2 \%) \\
128->132(12 \%)\end{array}$ & 4.294 & 288.8 & 0.079 \\
\hline S9 & $\begin{array}{l}122->129(39 \%) \\
122->131(2 \%) \\
124->130(6 \%) \\
125->129(34 \%) \\
125->130(9 \%)\end{array}$ & 4.321 & 286.9 & 0.0028 \\
\hline S10 & $\begin{array}{l}123->129(13 \%) \\
124->129(51 \%) \\
125->130(15 \%) \\
126->129(4 \%) \\
126->130(3 \%) \\
128->133(4 \%)\end{array}$ & 4.342 & 285.5 & 0.0021 \\
\hline S11 & $\begin{array}{l}121->129(3 \%) \\
122->129(39 \%) \\
123->129(9 \%) \\
124->129(3 \%) \\
124->130(14 \%) \\
125->129(19 \%) \\
126->129(2 \%) \\
127->130(2 \%)\end{array}$ & 4.372 & 283.6 & 0.0079 \\
\hline S12 & $\begin{array}{l}122->129(8 \%) \\
123->129(36 \%) \\
124->130(10 \%) \\
125->129(7 \%) \\
125->130(26 \%) \\
126->129(2 \%) \\
128->133(3 \%) \\
\end{array}$ & 4.394 & 282.2 & 0.0048 \\
\hline $\mathrm{S} 13$ & $\begin{array}{l}122->130(2 \%) \\
123->129(21 \%) \\
123->130(12 \%) \\
124->129(19 \%) \\
124->130(29 \%) \\
128->133(3 \%)\end{array}$ & 4.418 & 280.7 & 0.0216 \\
\hline S14 & $\begin{array}{l}123->130(20 \%) \\
125->130(4 \%) \\
126->133(4 \%) \\
127->130(4 \%) \\
127->133(5 \%) \\
128->133(50 \%)\end{array}$ & 4.426 & 280.1 & 0.0158 \\
\hline
\end{tabular}


Table S5. Lowest-energy vertical triplet excitations of 3d from TDDFT calculations at the ground state geometry in $\mathrm{CH}_{2} \mathrm{Cl}_{2}$ solution.

\begin{tabular}{|c|c|c|c|}
\hline State & monoexcitations & $\mathrm{AE} / \mathrm{eV}$ & $\lambda / \mathbf{n m}$ \\
\hline $\mathrm{T} 1$ & $\begin{array}{l}117->137(2 \%) \\
126->130(3 \%) \\
128->129(10 \%) \\
128->130(66 \%) \\
128->131(6 \%)\end{array}$ & 2.903 & 427.1 \\
\hline $\mathrm{T} 2$ & $\begin{array}{l}120->129(6 \%) \\
121->129(11 \%) \\
124->129(14 \%) \\
125->129(7 \%) \\
126->129(3 \%) \\
127->129(31 \%) \\
127->130(3 \%) \\
127->134(3 \%)\end{array}$ & 3.193 & 388.3 \\
\hline $\mathrm{T} 3$ & $\begin{array}{l}118->129(4 \%) \\
120->129(60 \%) \\
120->130(7 \%) \\
121->129(8 \%) \\
125->129(3 \%) \\
127->129(4 \%)\end{array}$ & 3.481 & 356.2 \\
\hline $\mathrm{T} 4$ & $\begin{array}{l}117->131(4 \%) \\
117->132(5 \%) \\
126->129(3 \%) \\
126->130(7 \%) \\
126->132(4 \%) \\
128->129(2 \%) \\
128->130(12 \%) \\
128->131(37 \%) \\
128->132(13 \%)\end{array}$ & 3.682 & 336.8 \\
\hline
\end{tabular}




\subsection{Complex (OC-6-33)-[PtCl $\left.2(\text { tpy })_{2}\right]$}

Table S6. Fragment contributions (\%; from atomic orbital contributions) to the frontier orbitals of (OC-6-33)-[ $\left[\mathrm{PtCl}_{2}(\text { tpy })_{2}\right]$ in $\mathrm{CH}_{2} \mathrm{Cl}_{2}$ solution.

\begin{tabular}{c|c|ccccc}
\hline energy (a.u.) & number & L1 & L2 & L3 & L4 & Pt \\
\hline-0.040 & 121 (LUMO+5) & 31 & 31 & 6 & 6 & 27 \\
-0.046 & 120 (LUMO+4) & 49 & 49 & 0 & 0 & 1 \\
-0.051 & 119 (LUMO+3) & 47 & 47 & 0 & 0 & 3 \\
-0.059 & $118($ LUMO+2) & 28 & 27 & 2 & 2 & 41 \\
-0.073 & $117($ LUMO+1) & 44 & 48 & 1 & 0 & 6 \\
-0.074 & $116($ LUMO) & 48 & 44 & 1 & 2 & 5 \\
-0.235 & $115($ HOMO) & 48 & 43 & 1 & 1 & 7 \\
-0.236 & 114 (HOMO-1) & 43 & 48 & 4 & 4 & 0 \\
-0.250 & 113 (HOMO-2) & 20 & 21 & 28 & 29 & 0 \\
-0.252 & $112($ HOMO-3) & 33 & 34 & 10 & 9 & 13 \\
-0.256 & 111 (HOMO-4) & 13 & 12 & 35 & 35 & 4 \\
-0.256 & $110($ HOMO-5) & 28 & 27 & 21 & 21 & 3 \\
\hline
\end{tabular}

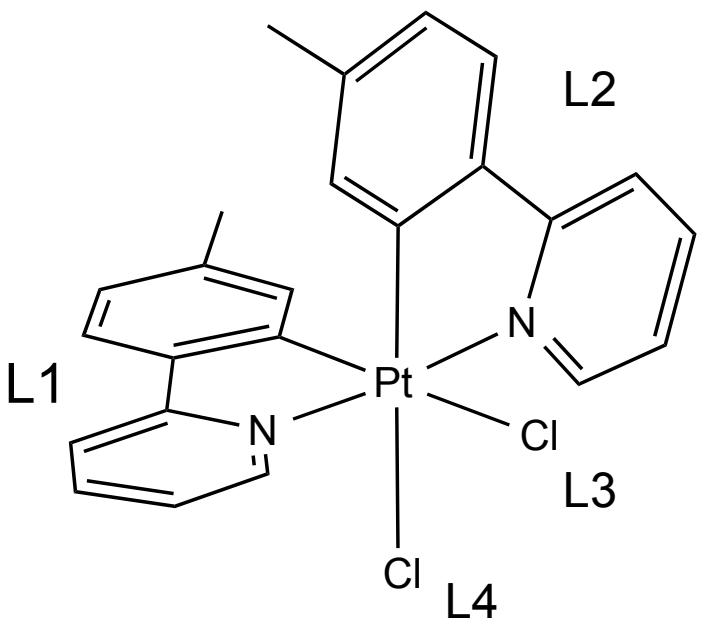

Figure S36. Ligand numbering in complex $(O C-6-33)-\left[\mathrm{PtCl}_{2}(\mathrm{tpy})_{2}\right]$. 


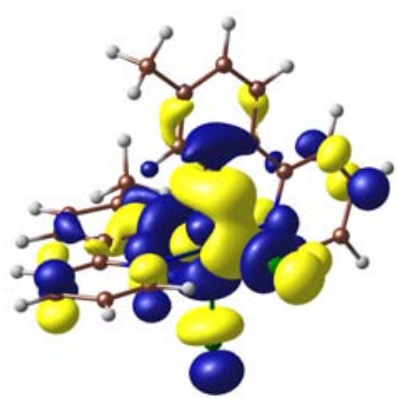

LUMO+5

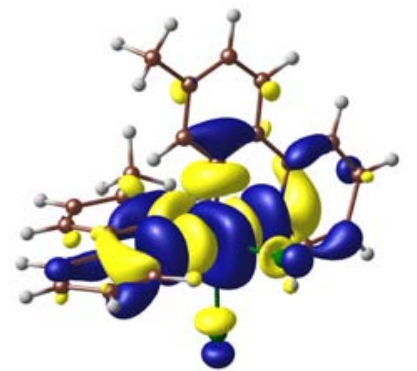

LUMO+2

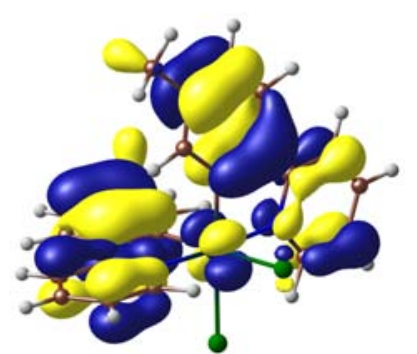

HOMO

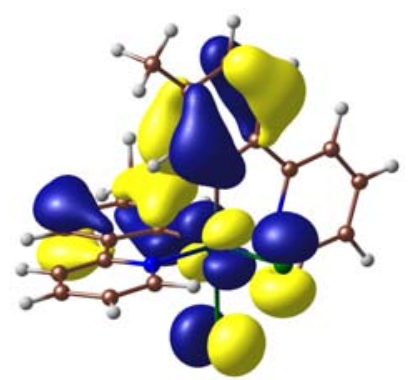

HOMO-3

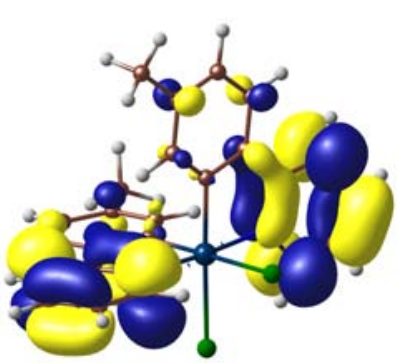

LUMO+4

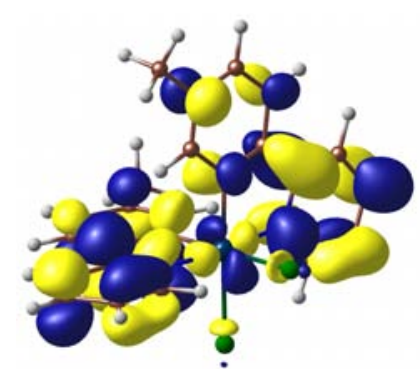

LUMO+1

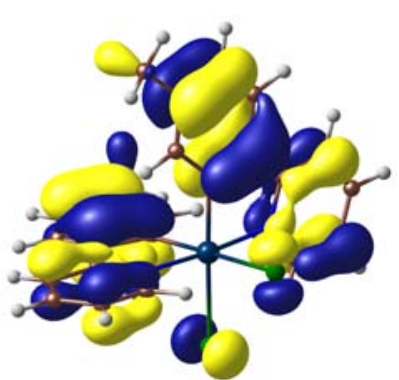

HOMO-1

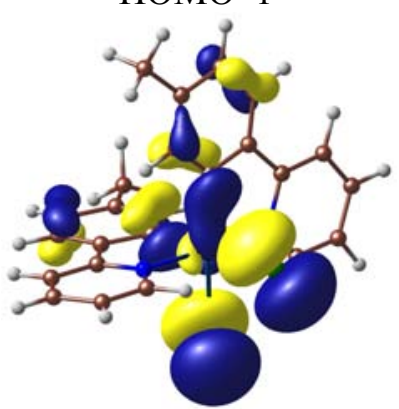

HOMO-4

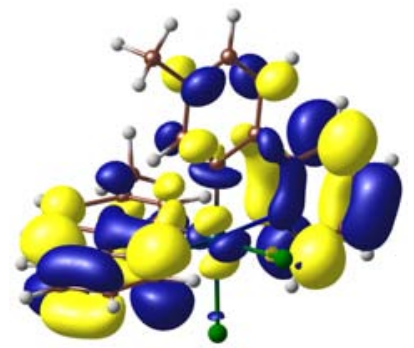

LUMO+3

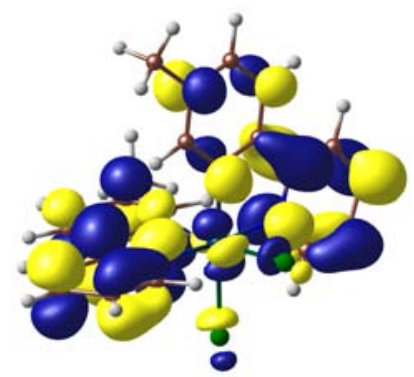

LUMO

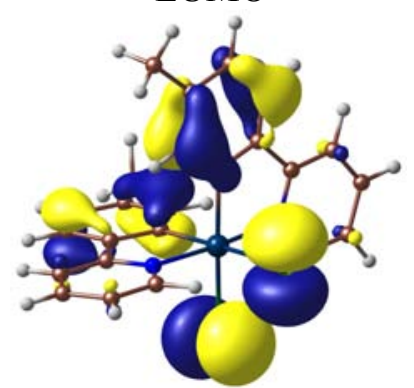

HOMO-2

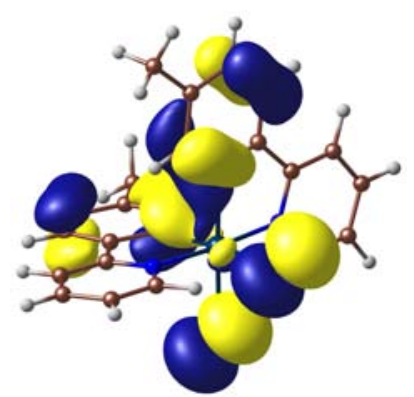

HOMO-5

Figure S37. Molecular orbital isosurfaces of $(\mathrm{OC}-6-33)-\left[\mathrm{PtCl}_{2}(\mathrm{tpy})_{2}\right]\left(0.03 \mathrm{e} \mathrm{bohr}^{-3}\right)$. 


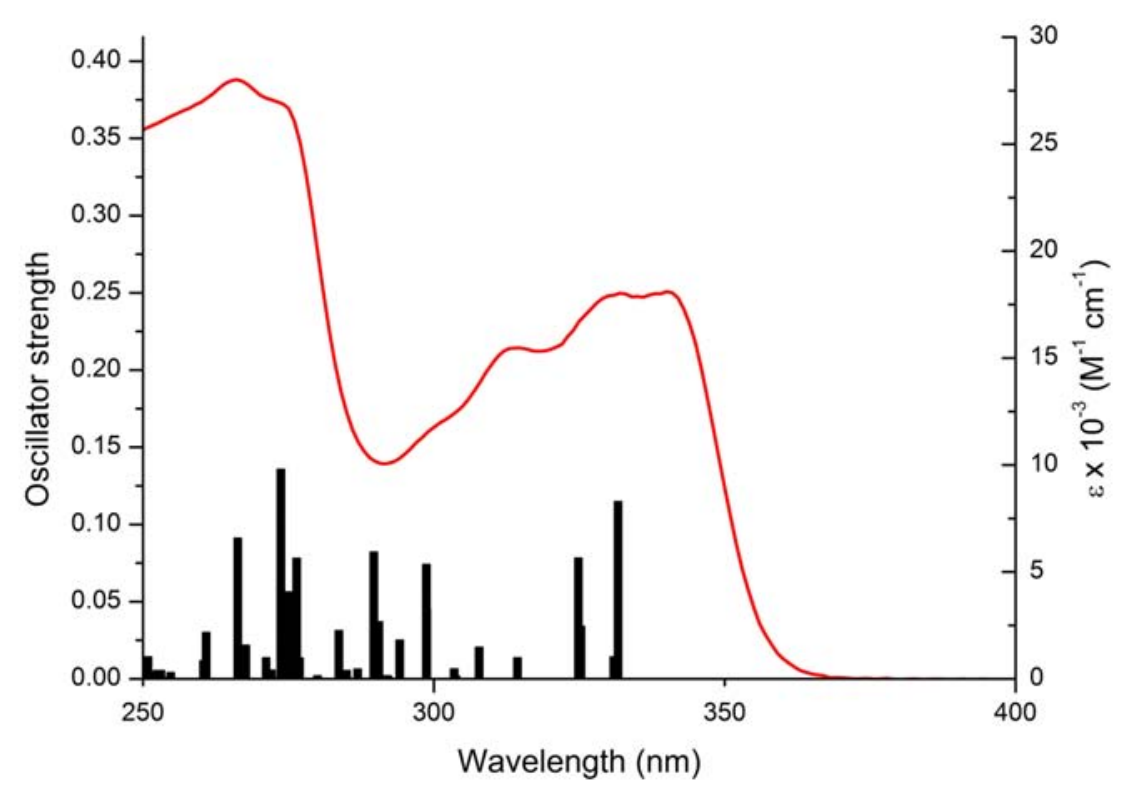

Figure S38. Calculated stick absorption spectrum of $(O C-6-33)-\left[\mathrm{PtCl}_{2}(\mathrm{tpy})_{2}\right]$ compared with the experimental spectrum in $\mathrm{CH}_{2} \mathrm{Cl}_{2}$ solution $\left(c a .1 \times 10^{-5} \mathrm{M}\right)$ at $298 \mathrm{~K}$.

Table S7. Selected vertical singlet excitations of $(O C-6-33)-\left[\mathrm{PtCl}_{2}(\mathrm{tpy})_{2}\right]$ from TDDFT calculations at the ground state geometry in $\mathrm{CH}_{2} \mathrm{Cl}_{2}$ solution.

\begin{tabular}{|c|c|c|c|c|}
\hline State & monoexcitations & $\mathrm{AE} / \mathrm{eV}$ & $\lambda / \mathbf{n m}$ & Oscillator strength \\
\hline S1 & $\begin{array}{l}112->116(6 \%) \\
115->116(83 \%) \\
115->121(2 \%)\end{array}$ & 3.738 & 331.7 & 0.1148 \\
\hline S2 & $\begin{array}{l}112->117(3 \%) \\
112->118(3 \%) \\
115->117(66 \%) \\
115->118(25 \%) \\
\end{array}$ & 3.747 & 330.9 & 0.0142 \\
\hline S3 & $\begin{array}{l}113->116(3 \%) \\
114->116(84 \%) \\
114->117(5 \%)\end{array}$ & 3.812 & 325.3 & 0.0341 \\
\hline S4 & $\begin{array}{l}113->117(2 \%) \\
114->116(6 \%) \\
114->117(75 \%) \\
114->118(13 \%)\end{array}$ & 3.817 & 324.9 & 0.0782 \\
\hline S5 & $\begin{array}{l}112->118(6 \%) \\
114->116(3 \%) \\
115->117(27 \%) \\
115->118(59 \%)\end{array}$ & 3.944 & 314.4 & 0.0136 \\
\hline S6 & $\begin{array}{l}114->117(14 \%) \\
114->118(78 \%) \\
115->116(3 \%)\end{array}$ & 4.028 & 307.8 & 0.0204 \\
\hline S7 & $\begin{array}{l}110->117(2 \%) \\
111->116(8 \%) \\
112->116(11 \%) \\
113->116(10 \%) \\
113->117(48 \%) \\
113->118(7 \%) \\
115->116(2 \%)\end{array}$ & 4.081 & 303.8 & 0.0017 \\
\hline S8 & $\begin{array}{l}110->116(4 \%) \\
111->117(6 \%)\end{array}$ & 4.085 & 303.5 & 0.0065 \\
\hline
\end{tabular}




\begin{tabular}{|c|c|c|c|c|}
\hline & $\begin{array}{l}112->117(10 \%) \\
113->116(55 \%) \\
113->117(9 \%)\end{array}$ & & & \\
\hline S9 & $\begin{array}{l}111->117(2 \%) \\
111->118(2 \%) \\
112->116(19 \%) \\
112->117(36 \%) \\
112->118(7 \%) \\
113->116(15 \%) \\
113->117(3 \%) \\
115->118(2 \%)\end{array}$ & 4.150 & 298.8 & 0.0451 \\
\hline S10 & $\begin{array}{l}112->116(44 \%) \\
112->117(15 \%) \\
112->118(3 \%) \\
113->116(6 \%) \\
113->117(10 \%) \\
115->119(3 \%)\end{array}$ & 4.151 & 298.7 & 0.0741 \\
\hline S11 & $\begin{array}{l}108->116(8 \%) \\
109->117(34 \%) \\
109->118(15 \%) \\
111->117(27 \%) \\
111->118(9 \%)\end{array}$ & 4.184 & 296.3 & 0.0003 \\
\hline S12 & $\begin{array}{l}108->117(15 \%) \\
108->118(4 \%) \\
109->116(55 \%) \\
110->117(3 \%) \\
111->116(9 \%) \\
112->116(4 \%)\end{array}$ & 4.215 & 294.1 & 0.0251 \\
\hline $\mathrm{S} 13$ & $\begin{array}{l}109->117(5 \%) \\
110->116(20 \%) \\
110->117(23 \%) \\
110->118(5 \%) \\
111->116(18 \%) \\
111->117(8 \%) \\
112->117(4 \%) \\
113->117(2 \%)\end{array}$ & 4.244 & 292.1 & 0.0016 \\
\hline S14 & $\begin{array}{l}109->117(6 \%) \\
110->116(19 \%) \\
110->117(19 \%) \\
110->118(4 \%) \\
111->116(13 \%) \\
111->117(14 \%) \\
111->118(2 \%) \\
112->117(6 \%) \\
113->116(2 \%) \\
\end{array}$ & 4.247 & 291.9 & 0.0019 \\
\hline S15 & $\begin{array}{l}109->117(14 \%) \\
109->118(3 \%) \\
110->116(38 \%) \\
111->117(14 \%) \\
112->117(3 \%) \\
112->118(5 \%) \\
114->119(12 \%) \\
114->121(5 \%)\end{array}$ & 4.267 & 290.6 & 0.037 \\
\hline
\end{tabular}


Table S8. Lowest-energy vertical triplet excitations of (OC-6-33)-[ $\left[\mathrm{PtCl}_{2}(\mathrm{tpy})_{2}\right]$ from TDDFT calculations at the ground state geometry in $\mathrm{CH}_{2} \mathrm{Cl}_{2}$ solution.

\begin{tabular}{|l|l|c|c|}
\hline State & monoexcitations & AE/eV & $\lambda / \mathbf{n m}$ \\
\hline T1 & $114->117(33 \%)$ & 2.866 & 432.6 \\
& $114->120(4 \%)$ & & \\
& $115->116(41 \%)$ & & \\
& $115->119(4 \%)$ & & 432.1 \\
\hline T2 & $113->116(2 \%)$ & 2.869 & \\
& $114->116(37 \%)$ & & \\
& $114->119(4 \%)$ & & \\
& $115->117(37 \%)$ & & 353.2 \\
& $115->120(4 \%)$ & & \\
\hline T3 & $112->117(8 \%)$ & 3.510 & \\
& $112->118(17 \%)$ & & \\
& $114->116(4 \%)$ & & \\
& $115->117(12 \%)$ & & \\
& $115->118(49 \%)$ & & \\
\hline T4 & $104->120(4 \%)$ & 3.615 & \\
& $105->119(3 \%)$ & & \\
& $110->117(3 \%)$ & & \\
& $112->116(10 \%)$ & & \\
& $113->120(3 \%)$ & & \\
& $114->120(17 \%)$ & & \\
& $115->116(15 \%)$ & & \\
& $115->119(28 \%)$ & & \\
\hline
\end{tabular}




\subsection{Complex 5d}

Table S9. Fragment contributions (\%; from atomic orbital contributions) to the frontier orbitals of $\mathbf{5 d}$ in $\mathrm{CH}_{2} \mathrm{Cl}_{2}$ solution.

\begin{tabular}{c|c|ccccc}
\hline energy (a.u.) & number & L1 & L2 & L3 & L4 & Pt \\
\hline-0.012 & 134 (LUMO+5) & 0 & 96 & 0 & 0 & 3 \\
-0.021 & 133 (LUMO+4) & 24 & 31 & 1 & 7 & 36 \\
-0.046 & $132($ LUMO+3) & 97 & 2 & 0 & 0 & 1 \\
-0.064 & 131 (LUMO+2) & 2 & 96 & 0 & 0 & 2 \\
-0.067 & $130($ LUMO+1) & 79 & 5 & 5 & 0 & 11 \\
-0.075 & $129($ LUMO) & 37 & 11 & 13 & 5 & 34 \\
-0.228 & $128($ HOMO) & 94 & 1 & 0 & 1 & 3 \\
-0.242 & 127 (HOMO-1) & 80 & 7 & 0 & 8 & 4 \\
-0.242 & $126($ HOMO-2) & 16 & 15 & 5 & 58 & 7 \\
-0.243 & $125($ HOMO-3) & 10 & 57 & 0 & 25 & 8 \\
-0.253 & 124 (HOMO-4) & 0 & 54 & 2 & 42 & 1 \\
-0.257 & 123 (HOMO-5) & 6 & 10 & 54 & 20 & 11 \\
\hline
\end{tabular}

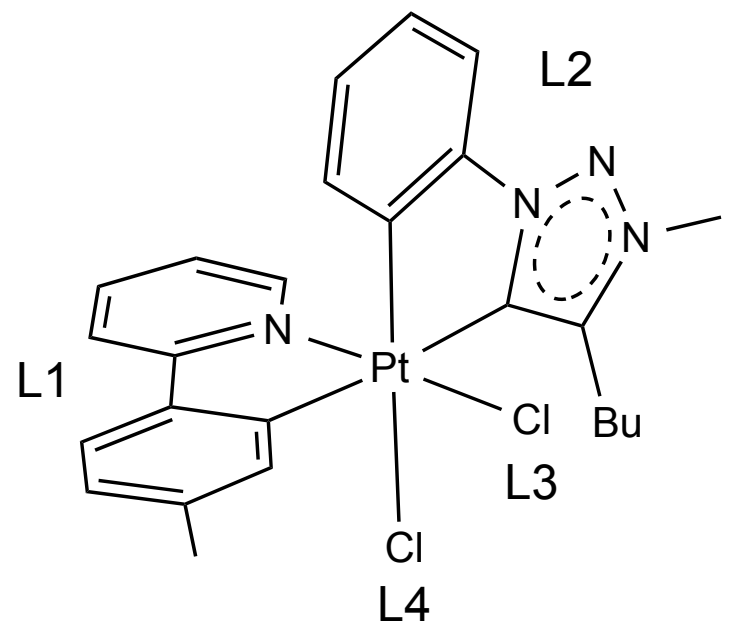

Figure S39. Ligand numbering in complex $\mathbf{5 d}$. 


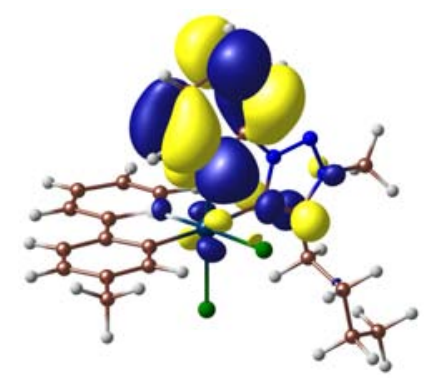

LUMO+5

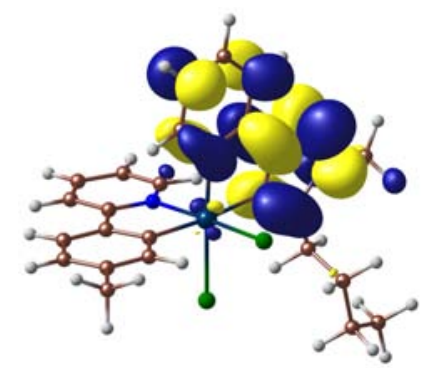

LUMO+2

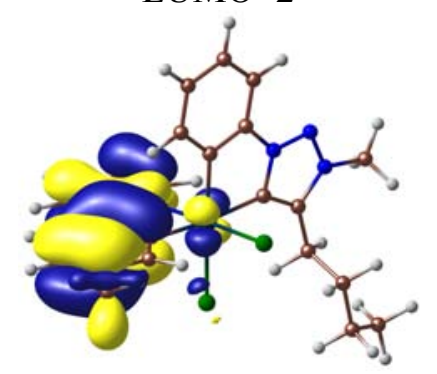

HOMO

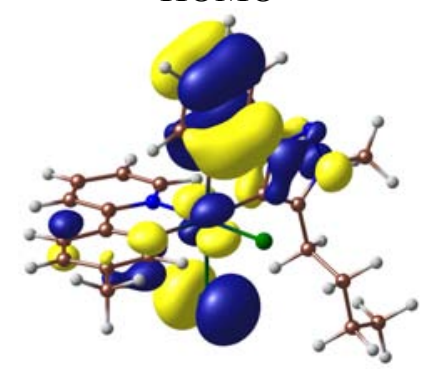

HOMO-3

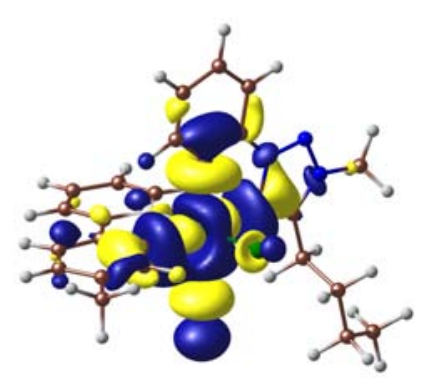

LUMO+4

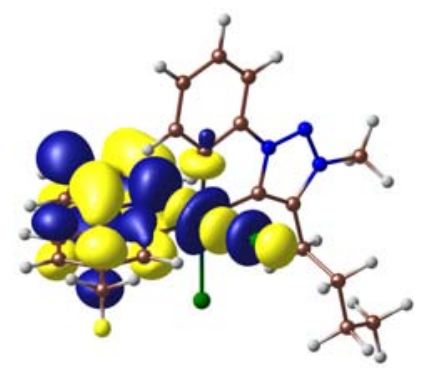

LUMO+1

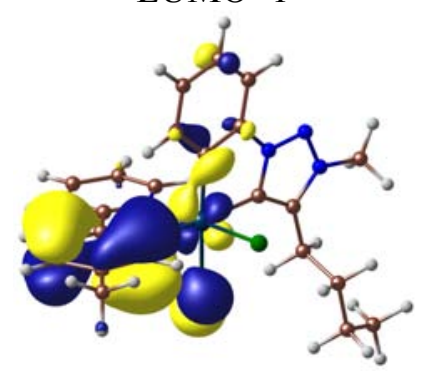

HOMO-1

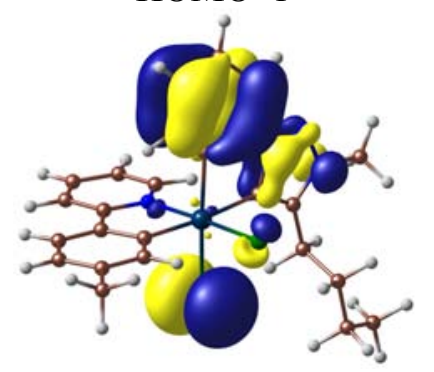

HOMO-4

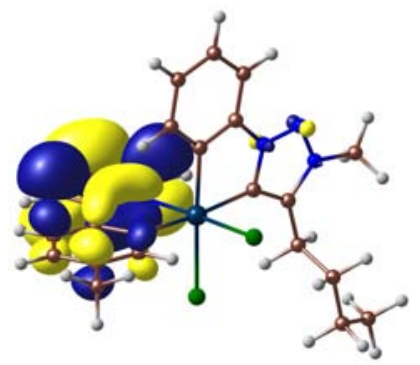

LUMO+3

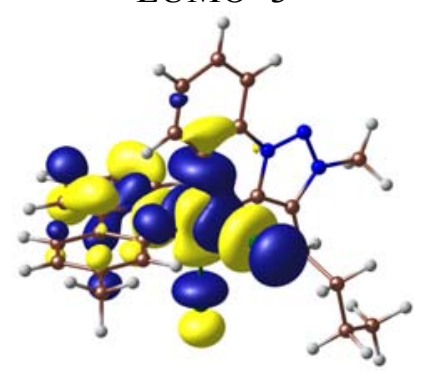

LUMO

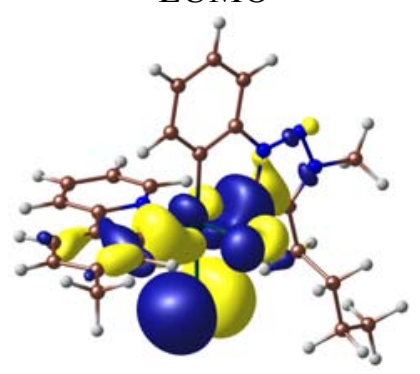

HOMO-2

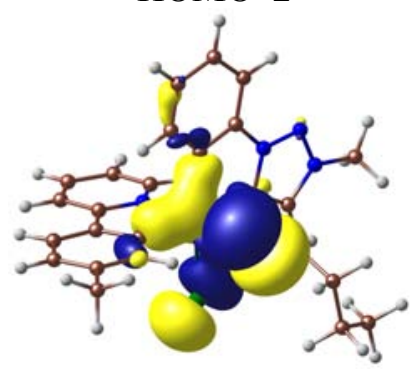

HOMO-5

Figure S40. Molecular orbital isosurfaces of $\mathbf{5 d}\left(0.03 \mathrm{e} \mathrm{bohr}^{-3}\right)$. 


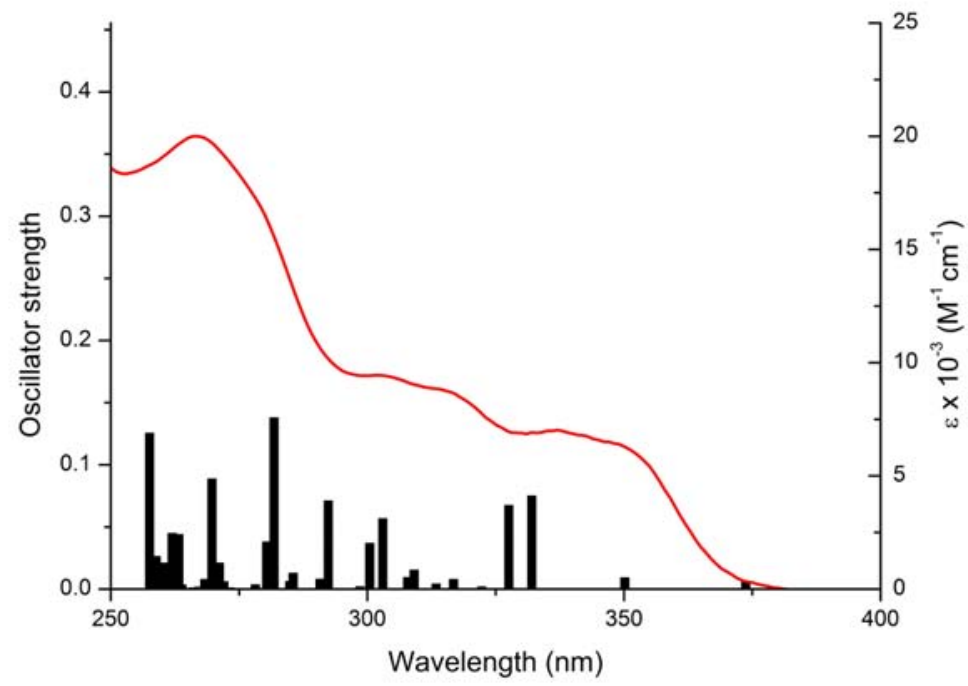

Figure S41. Calculated stick absorption spectrum of $\mathbf{5 d}$ compared with the experimental spectrum in $\mathrm{CH}_{2} \mathrm{Cl}_{2}$ solution $\left(\mathrm{ca} .1 \times 10^{-5} \mathrm{M}\right)$ at $298 \mathrm{~K}$.

Table S10. Selected vertical singlet excitations of 5d from TDDFT calculations at the ground state geometry in $\mathrm{CH}_{2} \mathrm{Cl}_{2}$ solution.

\begin{tabular}{|c|c|c|c|c|}
\hline State & monoexcitations & $\mathrm{AE} / \mathrm{eV}$ & $\lambda / \mathbf{n m}$ & Oscillator strength \\
\hline S1 & $\begin{array}{l}125->129(7 \%) \\
128->129(78 \%) \\
128->130(6 \%)\end{array}$ & 3.318 & 373.7 & 0.0064 \\
\hline S2 & $\begin{array}{l}123->129(5 \%) \\
125->129(27 \%) \\
125->130(3 \%) \\
126->129(46 \%) \\
126->130(4 \%) \\
127->129(7 \%)\end{array}$ & 3.506 & 353.6 & 0.0005 \\
\hline S3 & $\begin{array}{l}122->129(3 \%) \\
125->129(21 \%) \\
125->130(3 \%) \\
126->129(38 \%) \\
126->130(3 \%) \\
127->129(15 \%) \\
128->129(11 \%)\end{array}$ & 3.541 & 350.2 & 0.0092 \\
\hline S4 & $\begin{array}{l}122->129(3 \%) \\
125->129(6 \%) \\
126->129(5 \%) \\
127->129(15 \%) \\
128->130(63 \%)\end{array}$ & 3.734 & 332.0 & 0.075 \\
\hline S5 & $\begin{array}{l}123->129(2 \%) \\
125->129(12 \%) \\
125->130(2 \%) \\
127->129(45 \%) \\
127->130(6 \%) \\
128->129(7 \%) \\
128->130(16 \%) \\
\end{array}$ & 3.785 & 327.6 & 0.0674 \\
\hline S6 & $\begin{array}{l}121->129(4 \%) \\
122->129(15 \%) \\
122->130(2 \%) \\
123->129(49 \%)\end{array}$ & 3.847 & 322.3 & 0.0019 \\
\hline
\end{tabular}




\begin{tabular}{|c|c|c|c|c|}
\hline & $\begin{array}{l}123->130(7 \%) \\
124->129(3 \%) \\
125->129(7 \%) \\
127->129(5 \%)\end{array}$ & & & \\
\hline S7 & $\begin{array}{l}121->129(6 \%) \\
122->129(45 \%) \\
122->130(7 \%) \\
123->129(14 \%) \\
123->130(2 \%) \\
124->129(8 \%) \\
125->129(5 \%) \\
127->129(5 \%) \\
\end{array}$ & 3.914 & 316.8 & 0.0079 \\
\hline S8 & $128->131(96 \%)$ & 3.956 & 313.4 & 0.0043 \\
\hline S9 & $\begin{array}{l}126->129(5 \%) \\
126->130(86 \%) \\
128->130(2 \%)\end{array}$ & 4.011 & 309.1 & 0.0153 \\
\hline S10 & \begin{tabular}{|l|}
$121->129(6 \%)$ \\
$123->129(5 \%)$ \\
$124->129(73 \%)$ \\
$124->130(4 \%)$ \\
$125->129(4 \%)$
\end{tabular} & 4.028 & 307.8 & 0.0095 \\
\hline
\end{tabular}

Table S11. Lowest-energy vertical triplet excitations of 5d from TDDFT calculations at the ground state geometry in $\mathrm{CH}_{2} \mathrm{Cl}_{2}$ solution.

\begin{tabular}{|c|c|c|c|}
\hline State & monoexcitations & AE/eV & $\lambda / \mathbf{n m}$ \\
\hline T1 & \begin{tabular}{|l|}
$127->136(2 \%)$ \\
$128->129(35 \%)$ \\
$128->130(43 \%)$ \\
$128->132(8 \%)$
\end{tabular} & 2.826 & 438.8 \\
\hline $\mathrm{T} 2$ & \begin{tabular}{|l|}
$122->129(3 \%)$ \\
$123->129(11 \%)$ \\
$123->130(2 \%)$ \\
$125->129(17 \%)$ \\
$126->129(8 \%)$ \\
$128->129(25 \%)$ \\
$128->130(19 \%)$
\end{tabular} & 3.112 & 398.4 \\
\hline T3 & \begin{tabular}{|l|}
$120->131(3 \%)$ \\
$121->131(3 \%)$ \\
$121->134(3 \%)$ \\
$123->129(3 \%)$ \\
$124->131(24 \%)$ \\
$125->129(2 \%)$ \\
$125->131(28 \%)$ \\
$125->134(3 \%)$ \\
$126->129(11 \%)$ \\
\end{tabular} & 3.229 & 383.9 \\
\hline $\mathrm{T} 4$ & \begin{tabular}{|l|}
$119->129(2 \%)$ \\
$123->129(12 \%)$ \\
$123->130(3 \%)$ \\
$124->131(6 \%)$ \\
$125->129(10 \%)$ \\
$125->131(8 \%)$ \\
$126->129(32 \%)$ \\
$126->130(4 \%)$ \\
$127->129(5 \%)$ \\
\end{tabular} & 3.242 & 382.4 \\
\hline
\end{tabular}




\subsection{Supplementary computational data}

Table S12. Energies, free energies, enthalpies and entropies of the optimized structures in $\mathrm{CH}_{2} \mathrm{Cl}_{2}$

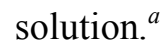

\begin{tabular}{|l|c|c|c|c|c|}
\hline Structure & $\mathbf{E}_{\mathbf{0}}{ }^{\boldsymbol{b}}$ & $\mathbf{Z P E}^{\boldsymbol{c}}$ & $\mathbf{G}^{\boldsymbol{d}}$ & $\mathbf{H}^{\boldsymbol{e}}$ & $\mathbf{S}^{\boldsymbol{f}}$ \\
\hline $\mathbf{3 d}\left(\mathrm{S}_{0}\right)$ & -2227.001644 & -2226.537181 & -2226.601643 & -2226.504893 & 203.627 \\
\hline $\mathbf{3 d}\left(\mathrm{T}_{1}\right)$ & -2226.899408 & -2226.439550 & -2226.506113 & -2226.406532 & 209.585 \\
\hline$(O C-6-33)-\left[\mathrm{PtCl}_{2}(\mathrm{tpy})_{2}\right]\left(\mathrm{S}_{0}\right)$ & -2075.820918 & -2075.441188 & -2075.500130 & -2075.413500 & 182.329 \\
\hline$(O C-6-33)-\left[\mathrm{PtCl}_{2}(\mathrm{tpy})_{2}\right]\left(\mathrm{T}_{1}\right)$ & -2075.719983 & -2075.344700 & -2075.404870 & -2075.316375 & 186.254 \\
\hline $\mathbf{5 d}\left(\mathrm{S}_{0}\right)$ & -2226.975464 & -2226.511417 & -2226.576405 & -2226.479072 & 204.853 \\
\hline $\mathbf{5 d}\left(\mathrm{T}_{1}\right)$ & -2226.897587 & -2226.436078 & -2226.504581 & -2226.402685 & 214.458 \\
\hline
\end{tabular}

${ }^{a}$ Thermal corrections from vibrational calculations at $298.15 \mathrm{~K} .{ }^{b}$ Electronic energy (Hartrees). ${ }^{c}$ Sum of electronic and zero-point energies (Hartrees). ${ }^{d}$ Free Energy (Hartrees). ${ }^{e}$ Enthalpy (Hartrees). ${ }^{f}$ Entropy (cal mol ${ }^{-1} \mathrm{~K}^{-1}$ ).

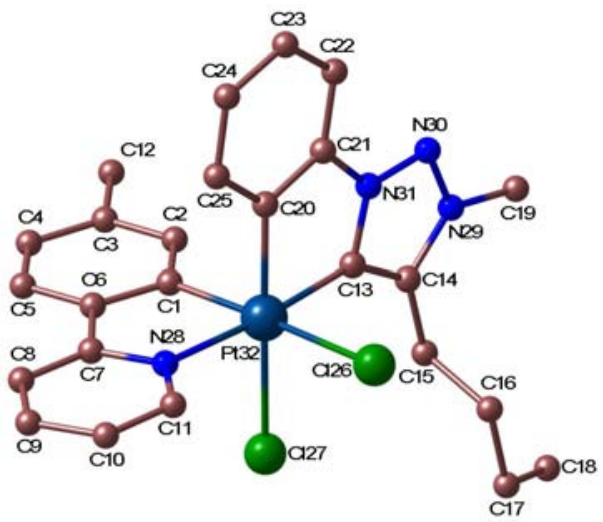

3d $\left(\mathrm{S}_{0}\right)$

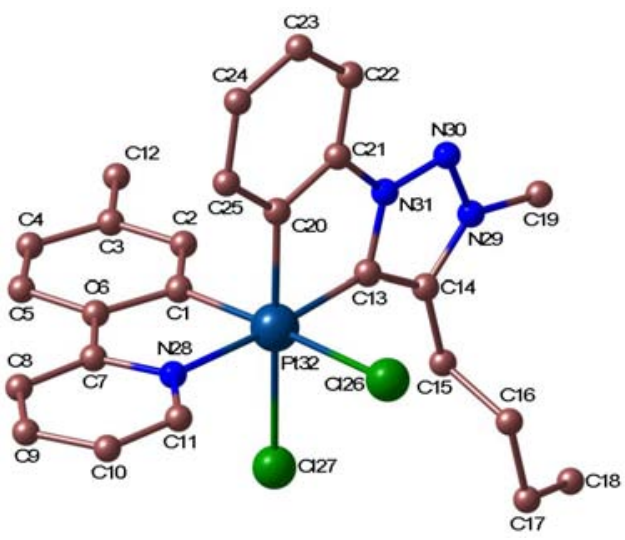

3d $\left(\mathrm{T}_{1}\right)$

Figure S42. Optimized structure of $\mathbf{3 d}$ in the ground state $\left(\mathrm{S}_{0}\right)$ and the lowest triplet state $\left(\mathrm{T}_{1}\right)$ with atom numbering (hydrogen atoms are omitted).

Table S13. Selected bond lengths $(\AA)$ for the optimized geometries of $\mathbf{3 d}$ in the ground state $\left(\mathrm{S}_{0}\right)$ and in the lowest triplet state $\left(T_{1}\right)$.

\begin{tabular}{|l|l|l|}
\cline { 2 - 3 } \multicolumn{1}{c|}{} & $\mathbf{S}_{\mathbf{0}}$ & $\mathbf{T}_{\mathbf{1}}$ \\
\hline C1-C2 & 1.391 & 1.371 \\
\hline C1-C6 & 1.413 & 1.481 \\
\hline C1-Pt32 & 2.033 & 2.020 \\
\hline C2-C3 & 1.405 & 1.421 \\
\hline C3-C4 & 1.402 & 1.441 \\
\hline C4-C5 & 1.390 & 1.361 \\
\hline C5-C6 & 1.404 & 1.455 \\
\hline C6-C7 & 1.466 & 1.389 \\
\hline C7-C8 & 1.401 & 1.441 \\
\hline C7-N28 & 1.360 & 1.415 \\
\hline
\end{tabular}

\begin{tabular}{|l|l|l|}
\cline { 2 - 3 } \multicolumn{1}{c|}{} & $\mathbf{S}_{\mathbf{0}}$ & $\mathbf{T}_{\mathbf{1}}$ \\
\hline C8-C9 & 1.390 & 1.371 \\
\hline C9-C10 & 1.397 & 1.420 \\
\hline C10-C11 & 1.389 & 1.404 \\
\hline C11-N28 & 1.342 & 1.324 \\
\hline C126-Pt32 & 2.546 & 2.541 \\
\hline C127-Pt32 & 2.522 & 2.544 \\
\hline N28-Pt32 & 2.135 & 2.117 \\
\hline C13-Pt32 & 2.013 & 2.018 \\
\hline C20-Pt32 & 2.045 & 2.045 \\
\hline
\end{tabular}



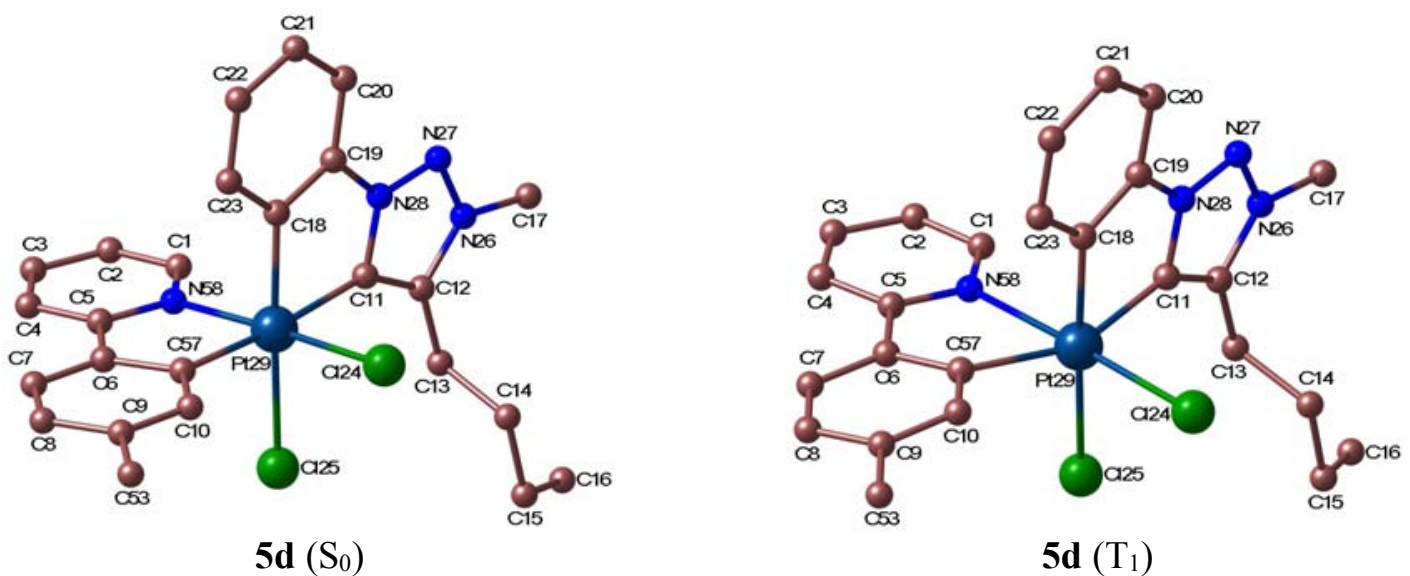

Optimized structure of $\mathbf{5 d}$ in the ground state $\left(\mathrm{S}_{0}\right)$ and the lowest triplet state $\left(\mathrm{T}_{1}\right)$

Figure S43. Optimized structure
with atom numbering (hydrogen atoms are omitted).

Table S14. Selected bond lengths $(\AA)$ and angles $\left(^{\circ}\right)$ for the optimized geometries of $\mathbf{5 d}$ in de ground state $\left(\mathrm{S}_{0}\right)$ and in the lowest triplet state $\left(\mathrm{T}_{1}\right)$.

\begin{tabular}{|l|l|l|}
\cline { 2 - 3 } \multicolumn{1}{c|}{} & $\mathbf{S}_{\mathbf{0}}$ & $\mathbf{T}_{\mathbf{1}}$ \\
\hline C1-C2 & 1.385 & 1.393 \\
\hline C1-N58 & 1.346 & 1.335 \\
\hline C2-C3 & 1.398 & 1.396 \\
\hline C3-C4 & 1.387 & 1.391 \\
\hline C4-C5 & 1.403 & 1.405 \\
\hline C5-C6 & 1.459 & 1.473 \\
\hline C5-N58 & 1.366 & 1.349 \\
\hline C6-C7 & 1.405 & 1.405 \\
\hline C6-C57 & 1.414 & 1.408 \\
\hline C7-C8 & 1.389 & 1.392 \\
\hline C8-C9 & 1.405 & 1.402 \\
\hline C9-C10 & 1.404 & 1.405 \\
\hline C9-C53 & 1.510 & 1.509 \\
\hline C10-C57 & 1.393 & 1.396 \\
\hline C11-C12 & 1.389 & 1.390 \\
\hline C11-C28 & 1.371 & 1.366 \\
\hline C11-Pt29 & 2.127 & 2.071 \\
\hline C124-Pt29 & 2.410 & 2.635 \\
\hline C125-Pt29 & 2.535 & 2.478 \\
\hline
\end{tabular}

\begin{tabular}{|l|l|l|}
\cline { 2 - 3 } \multicolumn{1}{c|}{} & $\mathbf{S}_{\mathbf{0}}$ & $\mathbf{T}_{\mathbf{1}}$ \\
\hline C26-C27 & 1.325 & 1.328 \\
\hline C27-C28 & 1.323 & 1.319 \\
\hline Pt29-C57 & 2.064 & 2.084 \\
\hline Pt29-N58 & 2.070 & 2.533 \\
\hline C11-Pt29-C18 & 80.0 & 79.0 \\
\hline C11-Pt29-C124 & 86.9 & 93.3 \\
\hline C11-Pt29-C125 & 95.3 & 98.5 \\
\hline C11-Pt29-C57 & 174.9 & 155.2 \\
\hline C11-Pt29-N58 & 98.0 & 87.0 \\
\hline C18-Pt29-C124 & 88.8 & 88.8 \\
\hline C18-Pt29-C125 & 175.2 & 177.2 \\
\hline C18-Pt29-C57 & 95.2 & 88.2 \\
\hline C18-Pt29-N58 & 91.1 & 93.7 \\
\hline C124-Pt29-C125 & 92.2 & 90.1 \\
\hline C124-Pt29-C57 & 94.8 & 107.7 \\
\hline C124-Pt29-N58 & 175.1 & 177.5 \\
\hline C125-Pt29-C57 & 89.4 & 94.6 \\
\hline C125-Pt29-N58 & 88.3 & 87.4 \\
\hline C57-Pt29-N58 & 80.3 & 72.6 \\
\hline
\end{tabular}


Table S15. Cartesian coordinates $(\AA)$ of the optimized structures at the B3LYP(6-31G**+LANL2DZ) level in $\mathrm{CH}_{2} \mathrm{Cl}_{2}$ solution.

\begin{tabular}{|c|c|c|c|}
\hline \multicolumn{4}{|c|}{$3 d\left(S_{0}\right)$} \\
\hline $\mathrm{C}$ & 35.252118033 & 10.969010706 & -3.979634320 \\
\hline $\mathrm{C}$ & 35.372003086 & 11.576303354 & -5.225488462 \\
\hline $\mathrm{C}$ & 34.241831721 & 11.855260993 & -6.012074379 \\
\hline $\mathrm{C}$ & 32.977665956 & 11.506591819 & -5.516026801 \\
\hline $\mathrm{C}$ & 32.843232286 & 10.902432217 & -4.271090015 \\
\hline $\mathrm{C}$ & 33.973324962 & 10.627137226 & -3.485055664 \\
\hline $\mathrm{C}$ & 33.908553032 & 9.979777975 & -2.171523229 \\
\hline $\mathrm{C}$ & 32.743216146 & 9.544312143 & -1.527480882 \\
\hline $\mathrm{C}$ & 32.831317437 & 8.936550273 & -0.280224228 \\
\hline $\mathrm{C}$ & 34.082367068 & 8.764693030 & 0.316662468 \\
\hline $\mathrm{C}$ & 35.207210306 & 9.213203822 & -0.363363451 \\
\hline $\mathrm{C}$ & 34.387958462 & 12.539582851 & -7.349516006 \\
\hline $\mathrm{C}$ & 38.264864291 & 11.253681290 & -3.833052095 \\
\hline $\mathrm{C}$ & 39.206209920 & 10.947573577 & -4.806668244 \\
\hline $\mathrm{C}$ & 39.506818158 & 9.681111341 & -5.541463029 \\
\hline $\mathrm{C}$ & 40.555559675 & 8.800565744 & -4.823489339 \\
\hline $\mathrm{C}$ & 40.857023170 & 7.490660120 & -5.567731811 \\
\hline $\mathrm{C}$ & 41.575038590 & 7.669084771 & -6.910880720 \\
\hline $\mathrm{C}$ & 41.027394061 & 12.349819149 & -5.913517445 \\
\hline $\mathrm{C}$ & 36.772775640 & 12.376761997 & -1.982981841 \\
\hline $\mathrm{C}$ & 37.740750580 & 13.220235553 & -2.547093314 \\
\hline $\mathrm{C}$ & 37.919956032 & 14.547353143 & -2.168859208 \\
\hline $\mathrm{C}$ & 37.081947078 & 15.059433734 & -1.177980942 \\
\hline $\mathrm{C}$ & 36.103663977 & 14.246283410 & -0.599165105 \\
\hline $\mathrm{C}$ & 35.945949048 & 12.912274873 & -0.998048194 \\
\hline $\mathrm{Cl}$ & 38.532616919 & 9.810588314 & -1.008098016 \\
\hline $\mathrm{Cl}$ & 36.819790446 & 8.156548565 & -3.727674834 \\
\hline $\mathrm{N}$ & 35.113142382 & 9.804929187 & -1.564738612 \\
\hline $\mathrm{N}$ & 39.924655403 & 12.105418319 & -4.984498820 \\
\hline $\mathrm{N}$ & 39.516080665 & 13.104533858 & -4.215767036 \\
\hline $\mathrm{N}$ & 38.521098635 & 12.567303484 & -3.535286116 \\
\hline $\mathrm{Pt}$ & 36.774953628 & 10.471632406 & -2.727549492 \\
\hline $\mathrm{H}$ & 35.347392164 & 12.302373805 & -7.818087378 \\
\hline $\mathrm{H}$ & 33.587941524 & 12.247820446 & -8.035888849 \\
\hline $\mathrm{H}$ & 34.339836512 & 13.630105293 & -7.237800021 \\
\hline $\mathrm{H}$ & 32.092136091 & 11.709020585 & -6.111884617 \\
\hline $\mathrm{H}$ & 31.851287945 & 10.640750416 & -3.916233218 \\
\hline $\mathrm{H}$ & 31.779163203 & 9.677507485 & -2.002632738 \\
\hline $\mathrm{H}$ & 31.930983362 & 8.596892459 & 0.221718908 \\
\hline $\mathrm{H}$ & 34.190574343 & 8.292456152 & 1.285936678 \\
\hline $\mathrm{H}$ & 36.212634326 & 9.113095869 & 0.030525091 \\
\hline $\mathrm{H}$ & 36.350929720 & 11.835756790 & -5.614712326 \\
\hline $\mathrm{H}$ & 38.689095002 & 15.155094481 & -2.632759564 \\
\hline $\mathrm{H}$ & 37.193844623 & 16.090719440 & -0.859729971 \\
\hline $\mathrm{H}$ & 35.454156743 & 14.650796659 & 0.171540738 \\
\hline $\mathrm{H}$ & 35.180910408 & 12.302725496 & -0.530343534 \\
\hline $\mathrm{H}$ & 39.836219513 & 9.922161791 & -6.557528115 \\
\hline $\mathrm{H}$ & 38.573576455 & 9.118566292 & -5.621027527 \\
\hline $\mathrm{H}$ & 41.487545323 & 9.365160879 & -4.684810742 \\
\hline $\mathrm{H}$ & 40.173944666 & 8.571720291 & -3.823062329 \\
\hline $\mathrm{H}$ & 39.918774868 & 6.941385989 & -5.719409303 \\
\hline $\mathrm{H}$ & 41.474642924 & 6.863113742 & -4.913773577 \\
\hline $\mathrm{H}$ & 41.809355683 & 6.698525488 & -7.360075050 \\
\hline $\mathrm{H}$ & 40.966445542 & 8.222030863 & -7.634412495 \\
\hline 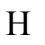 & 42.518745408 & 8.212831487 & -6.784559549 \\
\hline & 41.824059085 & 11.626969271 & $-5.7355761 \mathrm{c}$ \\
\hline
\end{tabular}

H $\quad 40.670252229$

H $\quad 41.392452331 \quad 13.358162534$

$-6.941409696$

$-5.729348681$

3d $\left(\mathrm{T}_{1}\right)$

$<S^{2}>=2.028427$

C 35.275494862

C 35.373473617

C 34.227704170

C 32.931019670

C 32.780459217

C 33.943898315

C 33.888146105

C 32.697859781

C 32.787678941

C 34.067247098

C $\quad 35.208120491$

C 34.345009854

C $\quad 38.276659281$

C $\quad 39.222052270$

C $\quad 39.524201896$

C 40.567662330

C 40.875198556

C 41.605257257

C 41.052633328

C $\quad 36.788911049$

C $\quad 37.759410534$

C 37.942227833

C 37.105844564

C $\quad 36.124610770$

C 35.962595006

$\mathrm{Cl} 38.535085108$

$\mathrm{Cl} \quad 36.805721316$

N 35.141263866

N 39.945403385

$\mathrm{N} \quad 39.537162240$

N 38.537263625

$\mathrm{Pt} \quad 36.779851481$

H 35.376585285

H 33.995991000

H 33.720870796

$\mathrm{H} \quad 32.052685027$

H 31.788623728

H 31.731669809

H 31.892444441

H 34.182857774

H 36.207256191

H 36.346367206

H 38.712829607

H 37.221086457

H $\quad 35.476158398$

H 35.194871480

H 39.859478493

H 38.590552938

H 41.498577399

H 40.178294715
10.973811137

11.583866937

11.872250334

11.532798740

10.942640745

10.623946234

10.016532246

9.594351408

8.983832790

8.773399054

9.204854992

12.497196971

11.248927108

10.946243807

9.681620939

8.797837782

7.491059331

7.674915457

12.348715709

12.366546018

13.209143733

14.533561091

15.042539274

14.229685024

12.898312690

9.771647218

8.143414968

9.800419697

12.102515941

13.098270040

12.559874206

10.466539445

12.773948258

11.810690828

13.396855339

11.760744909

10.706515136

9.762226435

8.662434077

8.288565429

9.069191250

11.847660314

15.141843936

16.071689538

14.632219363

12.287674425

9.924565844

9.120511918

9.361788168

8.564675830
$-3.987967745$

$-5.211806706$

$-6.001992653$

$-5.474179941$

$-4.257040771$

$-3.443280348$

$-2.195636566$

$-1.500920021$

$-0.276986793$

0.302242552

$-0.394079255$

$-7.358568059$

$-3.843560688$

$-4.814386803$

$-5.551732772$

$-4.829842652$

$-5.576977066$

$-6.912849365$

$-5.905525281$

$-1.982691751$

$-2.543678311$

$-2.15760502$

$-1.163624342$

$-0.58946895$

$-0.995642925$

$-1.037907539$

$-3.776332301$

$-1.574605847$

$-4.982416804$

$-4.209144388$

$-3.536422677$

$-2.738657642$

$-7.589340564$

$-8.142706135$

$-7.437506064$

$-6.072356611$

$-3.888607250$

$-1.961541981$

0.244432746

1.263839818

0.008612827

$-5.613537764$

$-2.618348395$

$-0.839665517$

0.183156607

$-0.533955915$

$-6.565458842$

$-5.636939728$

$-4.681436956$

$-3.833448682$ 
$\mathrm{H}$

41.486806169

$\mathrm{H} \quad 41.842701311$

$\mathrm{H} \quad 41.003701578$

H 42.548323824

H 41.845446206

H 40.699113345

H 41.421565176

(OC-6-33)-[ $\mathrm{PtCl}_{2}$ (tpy) $]$ ( $\left.\mathrm{S}_{0}\right)$

C $\quad-1.428427659$

C $\quad 1.939560519$

C $\quad-1.935287794$

C 2.949905576

C $\quad-2.939988207$

C $\quad 3.434734543$

C $\quad-3.423558695$

C 2.927104831

C $\quad-2.920024122$

C $\quad 1.918353133$

C $\quad-1.915530861$

C $\quad-1.373600763$

C $\quad 1.373372942$

C $\quad-1.745244109$

C 1.740725846

C $\quad-1.127057623$

C $\quad 1.118048867$

C $\quad-0.139968588$

C $\quad 0.130276077$

C 0.197564439

C $\quad-0.203915650$

H 3.365259259

H $\quad-3.351701606$

H 4.219044659

H $\quad-4.203390162$

$\mathrm{H} \quad 1.532011301$

H $\quad-1.531740537$

H $\quad-2.517913935$

H 2.512812592

H -1.414640951

H 1.402446601

$\mathrm{H} \quad 0.361490364$

H $\quad-0.374736837$

H 0.948841002

H $\quad-0.955484219$

N $\quad-0.401105709$

$\mathrm{N} \quad 0.399416416$

Pt $\quad-0.000135441$

$\mathrm{Cl}-1.813119728$

$\mathrm{Cl} \quad 1.813978445$

C $\quad-3.423166914$

H -4.454957148

H $\quad-2.812698609$

$\mathrm{H} \quad-3.379185924$

C $\quad 3.432284744$

H 4.483697722

H 2.864293957

H 3.330347605
6.942810217

6.860497754

6.706243909

8.231911184

8.217130470

11.621102548

12.272687120

13.354088980

0.129468534

0.115906631

$-1.131554645$

1.417403812

$-1.199881641$

1.621874070

$-0.037301082$

0.552790207

1.220199343

$-0.743386347$

1.285373734

$-0.945200522$

2.469624140

$-2.291438005$

3.819043297

$-3.628127597$

4.708664486

$-4.630432144$

4.245514148

$-4.293057543$

2.900052845

$-2.956059108$

$-2.158097785$

2.612895885

$-0.103321537$

0.723676273

2.257970540

$-1.948859614$

4.161439530

$-3.873454339$

5.754958637

$-5.667110134$

4.907378009

$-5.045509824$

2.463350987

$-2.613406097$

2.049135824

$-1.994498553$

0.021997092

$-0.260681938$

0.055964645

$-1.896401659$

$-1.733523288$

$-2.019406842$

$-2.838895180$

2.477301745

2.381584419

2.689345974

3.345954476
$-5.739085225$

$-4.920254977$

$-7.364485742$

$-7.639162655$

$-6.775550835$

$-5.729737944$

$-6.935367762$

$-5.712975778$

$-1.635288025$

$-1.638967241$

$-2.008575123$

$-1.835201908$

$-2.980343777$

$-2.793382714$

$-3.568623880$

$-3.539426854$

$-3.208009932$

$-3.353660318$

$-2.231463263$

$-2.391120351$

$-0.991739618$

$-1.324353184$

$-0.993661230$

$-1.517975478$

$-0.123316541$

$-0.783911180$

0.749911621

0.144481109

0.720153656

0.305221388

$-3.276583186$

$-2.957348094$

$-4.317233694$

$-4.276001436$

$-1.945660785$

$-2.241499506$

$-1.670751484$

$-2.236641638$

$-0.123047883$

$-0.931865551$

1.445730649

0.738197082

1.367203312

1.006302258

$-0.132520832$

$-0.416517621$

$-0.198657286$

1.569963607

1.590471988

$-4.187120502$

$-4.510700441$

$-5.090525705$

$-3.634003311$

$-3.872689249$

$-4.158357221$

$-4.787206254$

$-3.216308177$
(OC-6-33)-[ $\mathrm{PtCl}_{2}$ (tpy) $\left.)_{2}\right]\left(\mathrm{T}_{1}\right)$

$\left\langle S^{2}\right\rangle=2.026452$

C $\quad 1.423574029$

C -1.433322522

0.155829632

$-1.618315125$

C $\quad 1.936395251$

0.125757921

$-1.172684169$

1.424175291

$-1.950078351$

$-1.227031773$

1.621240713

$-0.078055555$

0.549175630

1.218157116

$-0.742896398$

1.285274704

$-0.938225833$

2.481548181

$-2.273285173$

3.827485251

$-3.655455882$

4.722821993

$-4.642903893$

4.268797090

$-4.285386563$

2.925995421

$-2.935188955$

$-2.185497218$

2.609178325

$-0.128612162$

0.715063691

2.262909510

$-1.937644162$

4.162995401

$-3.901208326$

5.766474540

$-5.684454871$

4.935514903

$-5.032437791$

2.495107930

$-2.614934440$

2.069649591

$-1.966528472$

0.036996601

$-0.246352357$

0.095492602

$-1.898764277$

$-1.734881464$

$-2.028673407$

$-2.838444628$

2.452236995

2.581232327

2.388970214

3.350730031

$-1.625868808$

$-2.003676455$

$-1.816886464$

$-3.024318914$

$-2.769715647$

$-3.591513363$

$-3.515082134$

$-3.217589040$

$-3.335333116$

$-2.227270623$

$-2.378156959$

$-0.977542681$

$-1.361310389$

$-0.976754567$

$-1.576897406$

$-0.112174624$

$-0.847065929$

0.751869111

0.128069130

0.719254050

0.312764865

$-3.331591986$

$-2.930831223$

$-4.352191736$

$-4.247199001$

$-1.953110891$

$-2.232786772$

$-1.646274602$

$-2.325729014$

$-0.109254875$

$-1.007937601$

1.442836293

0.734749682

1.359820850

1.040180831

$-0.127462781$

$-0.391009918$

$-0.192577425$

1.587200567

1.599567873

$-4.168766381$

$-4.505190579$

$-5.064038277$

$-3.610135454$

$-3.860035282$

$-3.614117746$

$-4.954461222$

$-3.538762048$

5d $\left(\mathrm{S}_{0}\right)$

C $\quad 35.307245867$

11.368915774

$-5.213948138$

$11.638449295 \quad-6.036516018$

11.349165556

$-5.565377078$

10.802096983

$-4.300068195$

10.543915637

$-3.497796892$ 


\begin{tabular}{|c|c|c|c|}
\hline & 7119 & 68 & -2.16 \\
\hline 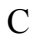 & 32.710930671 & 9.590764539 & -1.488969293 \\
\hline & 32.782090141 & 9.036579326 & -0.217020088 \\
\hline & 34.023103079 & 8.834650315 & 602019 \\
\hline & 35.192341642 & 9.214221377 & -0.269342621 \\
\hline & 38.293829337 & 11.205010424 & -3.907910356 \\
\hline & 39.269758234 & 10.955272841 & 49269 \\
\hline & 39.598524417 & 9.709549009 & -5.623864129 \\
\hline & 40.648042766 & 8.825316634 & -4.912342115 \\
\hline & 40.954313254 & 7.520295739 & -5.663333131 \\
\hline & 41.659807705 & 7.708470851 & -7.011756244 \\
\hline & 41.104720880 & 12.414968171 & -5.877888311 \\
\hline & 36.752938638 & 3875 & 989617 \\
\hline & 37.726784905 & 13.1 & 7238282 \\
\hline & 049325 & 14.4 & 60818 \\
\hline & 564019 & 15.0 & 43149 \\
\hline & 36.078625320 & 14.201360865 & -0.643683718 \\
\hline & 35.92620 & 12.8 & 64521 \\
\hline $\mathrm{Cl}$ & & & \\
\hline $\mathrm{Cl}$ & 36.80 & 8806 & 66878 \\
\hline$V$ & $39.9^{\prime}$ & 12.1 & 24367 \\
\hline$N$ & 39.534985099 & 13.091602036 & 534879 \\
\hline v & 38.5 & 12.5 & 21261 \\
\hline $\mathrm{Pt}$ & 36.725898315 & 92302 & -2.722830818 \\
\hline $\mathrm{H}$ & $32.0^{\prime}$ & & 520 \\
\hline H & 31.7 & 10. & 35235 \\
\hline 4 & 31.7 & 1819 & 12655 \\
\hline H & 31.867666140 & 8.7 & 92505 \\
\hline 1 & 36.153588093 & 9234 & 43601 \\
\hline 1 & 36.3 & & 25051 \\
\hline $\mathrm{H}$ & 38.6662 & 15. & 432922 \\
\hline $\mathrm{H}$ & 37.1 & 16.0 & 23737 \\
\hline & 35. & 1 & 324 \\
\hline $\mathrm{H}$ & 35.16 & 12.2 & 82093 \\
\hline 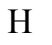 & 39.935859726 & 9057 & 443897 \\
\hline H & $38.6^{\circ}$ & 9.1 & 71360 \\
\hline $\mathrm{H}$ & 41.578434903 & 9.390906463 & -4.767366287 \\
\hline H & & & 7864 \\
\hline & 40.0 & & 193696 \\
\hline $\mathrm{H}$ & 41.5 & 6.8 & 3923 \\
\hline H & 41.89 & 6.74 & 972586 \\
\hline П & 41.039936620 & 3365 & 887991 \\
\hline $\mathrm{H}$ & 42.599163099 & 3151 & -6.891996236 \\
\hline $\mathrm{H}$ & 41.921414734 & 289771 & -5.674686841 \\
\hline H & 40.7 & 12.3 & -6.918218291 \\
\hline 4 & 41.4 & 13.4 & -5.680044707 \\
\hline H & 34.392321634 & 12.06 & -7.019302947 \\
\hline $\mathrm{C}$ & 34.099043242 & 8.197958378 & 1.776169445 \\
\hline $\mathrm{H}$ & 33.223425078 & 284645 & 2.383597107 \\
\hline $\mathrm{H}$ & 34.137850720 & 7.104326861 & 1.694593417 \\
\hline & 34.995998626 & 8.514982753 & 2.315645298 \\
\hline & 35.147540226 & 9.773353921 & -1.54473605 \\
\hline & 35.149180358 & 10.841749610 & -3.9854456 \\
\hline
\end{tabular}

C 33.023951397

C 32.856584910

C 33.986290017

C 33.933204384

C 32.742888585

C 32.729583640

C 33.901137830

C 35.090983191

C 38.338683618

C 39.185099188

C 39.505915541

C $\quad 40.726108634$

C $\quad 41.008696800$

C $\quad 41.446355648$

C 40.771445631

C 36.957568834

C 37.803367542

C 37.957765853

C $\quad 37.227219938$

C 36.378609341

C 36.245426064

Cl 38.819235933

$\mathrm{Cl} \quad 37.120807421$

N 39.790102986

N 39.387545681

N 38.517712430

Pt 36.979732259

H 32.156444197

H 31.860205693

H 31.813892894

$\mathrm{H} \quad 31.791846153$

H 36.006388339

H 36.410779084

H 38.633551923

H 37.323112253

$\mathrm{H} \quad 35.810250856$

H $\quad 35.578933616$

$\mathrm{H} \quad 39.668824503$

H $\quad 38.629618919$

H 41.618158810

H $\quad 40.535708649$

H 40.115136670

H 41.792968704

$\mathrm{H} \quad 41.689445124$

H $\quad 40.662973132$

H $\quad 42.338278768$

H $\quad 41.667105060$

H $\quad 40.341793604$

H 41.017891201

H $\quad 34.485374966$

C 33.895026045

H $\quad 32.937946970$

H $\quad 34.051857618$

H 34.692006405

C 35.115511932

N $\quad 35.221615622$
11.204662966

10.741654411

10.495537723

9.995681604

9.555942295

9.101649818

9.072851251

9.532298973

11.160914877

10.930133588

9.683177831

8.927878963

7.597720668

7.738542989

12.471859699

12.164287171

13.072631191

14.409267039

14.880796406

14.011125137

12.671929023

9.608384660

7.942023759

12.138849885

13.100530368

12.485626087

10.227311376

11.396736527

10.582017669

9.558390004

8.774085291

9.535430485

11.322009450

15.052542047

15.916782423

14.374613338

12.030618480

9.928942827

9.032481483

9.566472393

8.736029509

6.963040874

7.073268919

6.761401006

8.181020949

8.370371575

11.863906956

12.291602931

13.525830599

11.782711110

8.550640674

8.748885764

7.465198756

9.003291431

9.929517778

10.722261017
$-5.816149149$

$-4.515248037$

$-3.717757221$

$-2.332751514$

$-1.730041128$

$-0.414456176$

0.355690216

$-0.232689722$

$-3.708113098$

$-4.786058362$

$-5.544149873$

$-4.966713151$

$-5.681989540$

$-7.144678901$

$-6.059628152$

$-1.692359294$

$-2.351996462$

$-1.992503597$

$-0.903956304$

$-0.214794915$

$-0.602798102$

$-0.672119946$

$-3.400792760$

$-5.027066076$

$-4.205176929$

$-3.427609669$

$-2.454364261$

$-6.439582391$

$-4.121547471$

$-2.290895731$

0.025768909

0.350593136

$-5.781936993$

$-2.545477196$

$-0.595765279$

0.636550001

$-0.039434701$

$-6.598726416$

$-5.488519268$

$-5.015670789$

$-3.905357647$

$-5.621152254$

$-5.122840517$

$-7.574316216$

$-7.769331209$

$-7.230923151$

$-5.926839959$

$-7.046424921$

$-5.948389855$

$-7.314701117$

1.771261172

2.262806637

1.785652074

2.367475003

$-1.571104487$

$-4.210071972$

$<S^{2}>=2.006824$

$\begin{array}{llll}\text { C } & 35.386065866 & 11.168286846 & -5.457082859\end{array}$

$\begin{array}{llll}\text { C } & 34.312316861 & 11.423003457 & -6.306676932\end{array}$ 


\section{References}

(1) Sheldrick, G. M. A Short History of SHELX. Acta Crystallogr., Sect. A Found. Crystallogr. 2008, 64, 112-122.

(2) Spek, A. L. Single-Crystal Structure Validation with the Program PLATON. J. Appl. Crystallogr. 2003, 36, 7-13.

(3) van der Sluis, P.; Spek, A. L. BYPASS: An Effective Method for the Refinement of Crystal Structures Containing Disordered Solvent Regions. Acta Crystallogr. Sect. A Found. Crystallogr. 1990, 46, 194-201.

(4) Cardona, C. M.; Li, W.; Kaifer, A. E.; Stockdale, D.; Bazan, G. C. Electrochemical Considerations for Determining Absolute Frontier Orbital Energy Levels of Conjugated Polymers for Solar Cell Applications. Adv. Mater. 2011, 23, 2367-2371.

(5) Frisch, M. J.; Trucks, G. W.; Schlegel, H. B.; Scuseria, G. E.; Robb, M. A.; Cheeseman, J. R.; Scalmani, G.; Barone, V.; Mennucci, B.; Petersson, G. A.; Nakatsuji, H.; Caricato, M.; Li, X.; Hratchian, H. P.; Izmaylov, A. F.; Bloino, J.; Zheng, G.; Sonnenberg, J. L.; Hada, M.; Ehara, M.; Toyota, K.; Fukuda, R.; Hasegawa, J.; Ishida, M.; Nakajima, T.; Honda, Y.; Kitao, O.; Nakai, H.; Vreven, T.; Montgomery Jr., J. A.; Peralta, J. E.; Ogliaro, F.; Bearpark, M.; Heyd, J. J.; Brothers, E.; Kudin, K. N.; Staroverov, V. N.; Kobayashi, R.; Normand, J.; Raghavachari, K.; Rendell, A.; Burant, J. C.; Iyengar, S. S.; Tomasi, J.; Cossi, M.; Rega, N.; Millam, N. J.; Klene, M.; Knox, J. E.; Cross, J. B.; Bakken, V.; Adamo, C.; Jaramillo, J.; Gomperts, R.; Stratmann, R. E.; Yazyev, O.; Austin, A. J.; Cammi, R.; Pomelli, C.; Ochterski, J. W.; Martin, R. L.; Morokuma, K.; Zakrzewski, V. G.; Voth, G. A.; Salvador, P.; Dannenberg, J. J.; Dapprich, S.; Daniels, A. D.; Farkas, Ö.; Foresman, J. B.; Ortiz, J. V; Cioslowski, J.; Fox, D. J. Gaussian 09 (Revision A.02). Gaussian Inc.: Wallingford CT 2009.

(6) Becke, A. Density Functional Thermochemistry III The Role of Exact Exchange. J. Chem. Phys. 1993, 98, 5648-5652.

(7) Lee, C. T.; Yang, W. T.; Parr, R. G. Development of the Colle-Salvetti Correlation-Energy Formula into a Functional of the Electron Density. Phys. Rev. B 1988, 37, 785-789.

(8) Hariharan, P. C.; Pople, J. A. Influence of Polarization Functions on Molecular-Orbital Hydrogenation Energies. Theor. Chim. Acta 1973, 28, 213-222.

(9) Francl, M. M.; Pietro, W. J.; Hehre, W. J.; Binkley, J. S.; Gordon, M. S.; Defrees, D. J.; Pople, J. A. Self-Consistent Molecular Orbital Methods. XXIII. A Polarization-Type Basis Set for Second-Row Elements. J. Chem. Phys. 1982, 77, 3654-3665.

(10) Hay, P. J.; Wadt, W. R. Ab Initio Effective Core Potentials for Molecular Calculations-Potentials for K to Au Including the Outermost Core Orbitals. J. Chem. Phys. 1985, 82, 299-310.

(11) Escudero, D.; Thiel, W. Exploring the Triplet Excited State Potential Energy Surfaces of a Cyclometalated Pt(II) Complex: Is There Non-Kasha Emissive Behavior? Inorg. Chem. 2014, 53, 11015-11019.

(12) Tomasi, J.; Mennucci, B.; Cammi, R. Quantum Mechanical Continuum Solvation Models. Chem. Rev. 2005, 105, 2999-3093.

(13) Glendening, E. D.; Badenhoop, J. K.; A. E. Reed; Carpenter, J. E.; Bohmann, J. A.; Morales, C. M.; Weinhold, F. NBO 5.9. Theoretical Chemistry Institute, University of Wisconsin: Madison, WI 2009. 